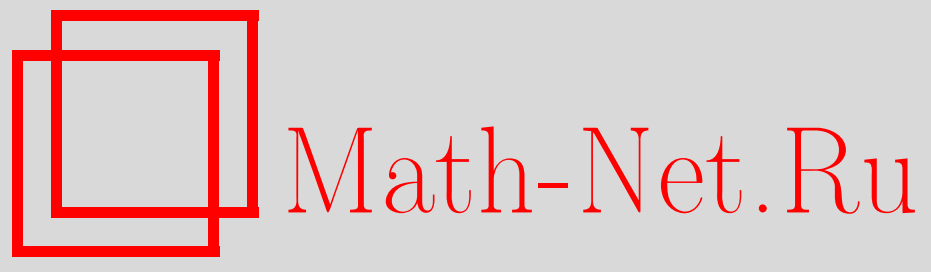

С. М. Дудаков, М. А. Тайцлин, Трансляционные результаты для языков запросов в теории баз данных, УМH, 2006, том 61, выпуск 2, 3-66

DOI: https://doi.org/10.4213/rm1713

Использование Общероссийского математического портала Math-Net.Ru подразумевает, что вы прочитали и согласны с пользовательским соглашением http://www . mathnet.ru/rus/agreement

Параметры загрузки:

IP : 3.80 .181 .102

26 апреля 2023 г., 12:41:31

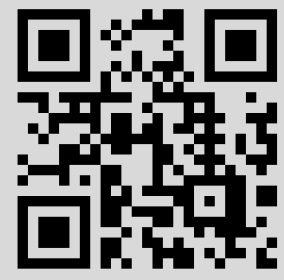




\title{
Трансляционные результаты для языков запросов в теории баз данных
}

\begin{abstract}
С. М. Дудаков, М. А. Тайцлин
В этом обзоре мы излагаем трансляционные результаты, полученные, главным образом, участниками тверского семинара "Теоретические основы информатики". В центре нашего внимания так называемые относительные свойства изолированности и псевдоконечной однородности и универсумы без независимой формулы. Для последних мы доказываем теорему Болдвина-Бенедикта о сводимости. Для сводимых теорий мы доказываем теорему Дудакова об ограниченности. Для сводимых и ограниченных теорий мы доказываем теорему об относительной изолированности и, как следствие, получаем для сводимых теорий трансляционную теорему. Мы также замечаем, что сводимость равносильна относительной изолированности. С другой стороны, мы приводим теоремы Дудакова, которые показывают, что для эффективно сводимых теорий, в которых существует эффективная почти неразличимая последовательность, возможна эффективная трансляция локально генерических запросов, использующих, кроме упорядочения и имен хранящихся таблиц, также и отношения и операции универсума, в запросы, которые эти отношения и операции универсума уже не используют. Мы приводим также пример Дудакова такого обогащения арифметики Пресбургера, для которого трансляционная теорема не имеет места, но элементарная теория которого разрешима. Это дает отрицательный ответ на некоторые открытые вопросы.
\end{abstract}

Библиография: 23 названия.

\section{СОДЕРЖАНИЕ}

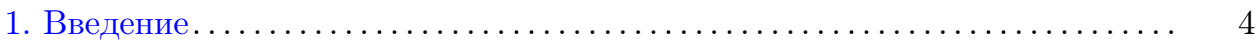

2. Предварительные сведения из теории множеств ................ 7

3. Предварительные сведения из теории моделей.................. 10

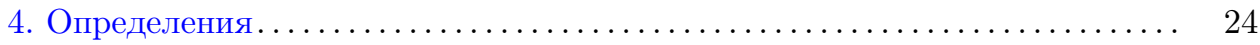

5. Критерий транслируемости расширенного запроса в ограниченный ... 30

6. Относительные свойства псевдоконечной однородности и изолирован-

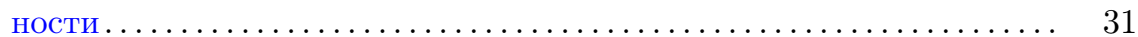

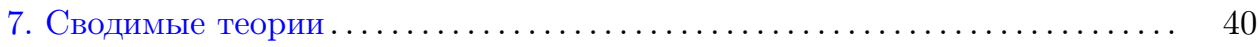

8. Ограниченность сводимых теорий..................... 48

Работа выполнена при поддержке Российского фонда фундаментальных исследований (гранты 04-01-00015 и 04-01-00565).

(C) С. М. ДудАков, М. А. ТАйцлин, 2006 
9. Активные запросы. Трансляция локально генерического запроса в активный для сводимых теорий....................... 58

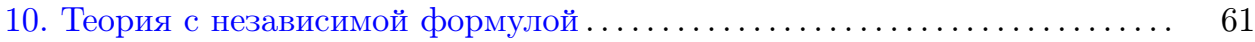

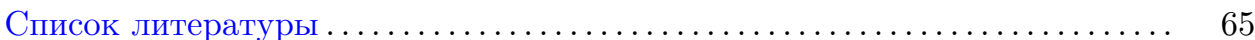

\section{1. Введение}

Типичной моделью базы данных со времен Кодда является реляционная модель, в которой база данных мыслится как собрание конечного числа конечных таблиц (см. [1], [2]). Эта модель реализуется в большинстве существующих средств управления базами данных и в предлагаемых языках запросов. При этом в качестве языка запросов обычно предлагается та или иная стилизация языка логики предикатов первого порядка. Эта традиция тоже восходит к Кодду, который в качестве языка запросов предложил использовать язык реляционных выражений, практически эквивалентный языку логики предикатов первого порядка.

Обычно при этом удобно предполагать, что элементы хранящихся таблиц выбираются из фиксированного множества, называемого универсумом. Например, в качестве такового можно взять множество натуральных чисел, множество всех слов некоторого конечного алфавита или какое-то другое множество. Это множество должно быть бесконечным. Оно может быть снабжено своими собственными отношениями и операциями. Они составляют сигнатуру универсума. Эти отношения и операции обычно по своей природе не могут быть заданы конечными таблицами.

Итак, базы данных предназначены для хранения текущей информации о как-то структурированной предметной области. В каждый момент времени эта информация является конечной и представляет собой конечный набор конечных таблиц [1], [2]. Обычно число таблиц и устройство каждой таблицы не меняются с течением времени, но меняются строки таблиц. Могут добавляться новые строки и удаляться некоторые старые. Строки хранящихся таблиц представляют собой конечные последовательности элементов. Число элементов каждой последовательности фиксировано для фиксированной таблицы. Устройство таблицы практически и есть число элементов в каждой строке этой таблицы. Более формально (или более научно), каждая таблица - это конечноместное конечное отношение, а сама база данных - это конечный набор конечноместных конечных отношений. Для удобства разговора о базе данных каждому ее отношению приписывают некоторое имя с указанием числа аргументов (или местности) этого имени отношения. Схема (или сигнатура) базы данных и есть конечная последовательность этих имен отношений с указанием местности каждого имени. В каждый момент времени именам отношений из этой схемы присвоены некоторые отношения соответствующих местностей. Это - состояние базы данных в данный момент.

Состояние называется конечным, если все его отношения конечны (каждая таблица содержит конечное множество строк). Иногда удобно рассматривать не произвольные состояния базы данных, а ограниченные какими-то условиями. Типичным ограничением является условие, что элементы всех строк всех 
таблиц выбраны из фиксированного подмножества $I$ универсума. Другими словами, каждому имени отношения из рассматриваемой схемы базы данных поставлено в соответствие отношение той же местности на множестве $I$. В этом случае говорят, что рассматриваемое состояние базы данных является состоянием над $I$.

Мы будем рассматривать только линейно упорядоченные универсумы. Так называют универсумы, для которых среди отношений универсума есть бинарное отношение, которое является отношением линейного порядка (тотальным, транзитивным и антисимметричным отношением). Мы будем использовать < как имя для этого линейного порядка.

Итак, запросами являются формулы логики предикатов первого порядка.

Для линейно упорядоченных универсумов мы будем рассматривать локально генерические запросы, которые сохраняются при любых сохраняющих упорядочение отображениях конечных подмножеств универсума в универсум. Грубо говоря, ответ на такой запрос основывается на хранящейся информации, но не зависит от способа кодировки этой информации при хранении. Более точное определение приводится в разделе 4.

В языке запросов, кроме имен хранящихся таблиц, можно использовать и имена отношений и операций самого универсума. Другими словами, в языке запросов можно рассматривать как хранящуюся информацию, так и общие знания об универсуме. Например, язык SQL, используемый в системе Oracle и в других подобных системах, разрешает использовать как имена хранящихся таблиц, так и отношение сравнения и арифметические операции над числами.

Запросы, в которых используются как имена отношений из схемы базы данных, так и имена отношений и операций самого универсума, называются расширенными. Запросы, в которых используется только < и имена отношений из схемы базы данных, называются ограниченными. Утверждение, что каждый локально генерический расширенный запрос эквивалентен для конечных состояний базы данных некоторому ограниченному запросу, называется транслячионной теоремой или трансляционным результатом для рассматриваемого универсума. ${ }^{1}$

Расширяет ли использование общих знаний выразительные возможности языка запросов?

Обычно рассматривают упорядоченные универсумы. Более специальный вопрос: расширяет ли использование упорядочения универсума выразительные возможности языка запросов?

Понятно, что надо рассматривать только запросы, не зависящие от упорядочения универсума, другими словами, не меняющиеся при любой перестановке элементов универсума. Такие запросы называют =-генерическими.

Ответ получен Ю.Ш. Гуревичем (см. [3]). Ответ не зависит от универсума и является положительным. Даже если мы ничего не знаем о порядке, мы всегда можем предложить =-генерический запрос, который нельзя записать без использования отношения порядка (см. [4]).

Поразительно, но во многих случаях имеет место трансляционная теорема: использование других общих знаний дополнительно к упорядочению уже не

\footnotetext{
${ }^{1}$ Англ. collapse theorem, collapse result.
} 
расширяет выразительные возможности языка запросов. Подробнее об этом можно прочитать в [3]-[12].

Для других теорий, например, элементарной арифметики или теории наследственно конечных множеств, это не так, использование знаний этих универсумов увеличивает возможности языка первого порядка. Существуют и разрешимые примеры таких теорий (см. [13]). А в некоторых случаях (см. [14]) увеличение выразительной возможности зависит от сигнатуры базы данных.

Вопрос, изучению которого посвящена эта работа, состоит в описании тех универсумов, для которых верна трансляционная теорема.

Критерий того, что некоторый запрос эквивалентен ограниченному, был найден в [3]. Там же были предложены условия псевдоконечной однородности и изолированности, выполнимость в универсуме одного из которых гарантирует справедливость трансляционной теоремы для этого универсума. С другой стороны, в [5] трансляционная теорема доказана для универсумов без независимой формулы. Как связаны между собой эти условия?

В [15] были предложены более ограниченные понятия $(M, I)$-псевдоконечной однородности и $(M, I)$-изолированности, в которых рассматриваются состояния не над любыми неразличимыми последовательностями, а над некоторой фиксированной неразличимой последовательностью. Это во многих случаях позволяет значительно облегчить доказательство трансляционной теоремы (см., например, [10], [7]). Мы приводим упрощенное и более аккуратное изложение результатов [15].

В частности, оказывается, что универсумы без независимой формулы удовлетворяют условию изолированности и, значит, справедливость трансляционной теоремы для них является следствием наших теорем.

Формулы логики предикатов первого порядка сигнатуры, состоящей только из <, называются порядковыми. Формулы логики предикатов первого порядка сигнатуры $L$ называются $L$-формулами. Пусть $(M, I)$ - обогащение системы $M$ сигнатуры $L$ до системы сигнатуры $(L, P)$ путем добавления подмножества $I$ как интерпретации для одноместного отношения $P$. Говорят, что система $(M, I)$ является сводимой, если

для каждой $L$-формулы $\phi(\bar{x}, \bar{y})$ существует такая порядковая формула $\psi(\bar{w}, \bar{y})$, что для каждой последовательности $\bar{m}$ элементов $M$ существует последовательность $\bar{c}_{\bar{m}} \in I$, для которой

$$
(\forall \bar{y} \in P)\left(\psi\left(\bar{c}_{\bar{m}}, \bar{y}\right) \leftrightarrow \phi(\bar{m}, \bar{y})\right)
$$

Универсум $U$ называется сводимым, если существует такая сводимая система $(M, I)$, что $U$ и $M$ элементарно эквивалентны, а $I$ является бесконечной неразличимой последовательностью в $M$.

$(L, P)$-формула называется $P$-ограниченной, если она не содержит $P$ или имеет вид

$$
(\forall x \in P) \Psi \quad \text { или } \quad(\exists x \in P) \Psi,
$$

где $\Psi$ является $P$-ограниченной формулой. Система $(M, I)$ сигнатуры $(L, P)$ называется ограниченной, если каждая $(L, P)$-формула эквивалентна в $(M, I)$ некоторой $P$-ограниченной формуле. 
Доказательство трансляционной теоремы для универсумов без независимой формулы в [5] состоит из двух частей. Сначала доказывается, что такой универсум является сводимым. Затем доказывается, что такие универсумы ограничены, для этого снова используется отсутствие независимой формулы.

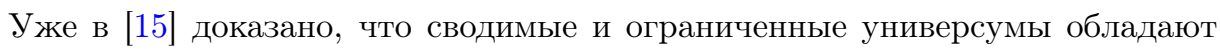
трансляционной теоремой. Здесь мы доказываем теорему Дудакова о том, что каждый сводимый универсум является ограниченным. Значит, для всех сводимых универсумов справедлива трансляционная теорема. Отсюда получается более простое доказательство трансляционной теоремы для универсумов без независимой формулы. Попутно мы замечаем, что относительная изолированность равносильна сводимости.

Активной областью состояния базы данных называется совокупность всех элементов универсума, входящих в хотя бы одну строку хотя бы одной таблицы. Запрос называется активным, если все его кванторы ограничены активной областью. Мы доказываем теорему Дудакова, что в каждом сводимом универсуме каждый локально генерический для конечных состояний расширенный запрос эквивалентен для конечных состояний некоторому активному запросу. Для сводимых универсумов, в которых можно эффективно строить неразличимые для заданной формулы последовательности, из этого получается эффективная трансляция расширенных запросов в ограниченные, чего принципиально невозможно было достичь старыми методами.

В [10] указано много примеров обогащений арифметики Пресбургера одной одноместной операцией с разрешимой элементарной теорией. Там же замечено, что для всех этих примеров имеет место трансляционная теорема. Принципиально другой пример обогащения арифметики Пресбургера одной одноместной операцией с разрешимой элементарной теорией предложен в [16]. Мы предлагаем некоторое усовершенствование этого примера и приводим теорему Дудакова о том, что для этого примера трансляционная теорема ложна. Это опровергает известную гипотезу (см. [5], [4], [10]), что все обогащения арифметики Пресбургера с разрешимой элементарной теорией обладают трансляционной теоремой.

\section{2. Предварительные сведения из теории множеств}

Мы предполагаем, что читатель знаком с начальными сведениями из наивной теории множеств.

Ординал мы отождествляем с множеством всех меньших ординалов, а кардинал - с наименьшим ординалом соответствующей мощности. Как обычно, $\aleph$ обозначает бесконечный кардинал. Бесконечные кардиналы можно занумеровать ординалами. В частности, אֻ обозначает наименьший бесконечный кардинал, $\aleph_{\alpha+1}-$ наименьший из кардиналов, больших $\aleph_{\alpha}$, а для предельного ординала $\alpha$ через $\aleph_{\alpha}$ обозначается наименьший из кардиналов, больших всех кардиналов $\aleph_{\beta}$ для всех $\beta<\alpha$.

Вместе с тем, наименьший бесконечный кардинал является и наименьшим бесконечным ординалом и обозначается также через $\omega$. Все конечные ординалы являются кардиналами и называются натуральными числами. Таким образом, $\omega$ и $\aleph_{0}$ обозначают множество натуральных чисел. 
Если мощность множества $A$ есть множества всех подмножеств множества $A$.

Через $\kappa^{+}$для кардинала $\kappa$ обозначается наименьший из кардиналов, больших $\kappa$. Через $\aleph_{\alpha}^{*}$ обозначается $\sum_{\beta<\alpha} 2^{\aleph_{\beta}}$. Мы полагаем $\beth_{0}=\aleph_{0}$ и $\beth_{\xi+1}=2^{\beth_{\xi}}$. Для предельного $\xi$ пусть

$$
I_{\xi}=\bigcup_{\zeta<\xi} I_{\zeta} .
$$

Пусть $\alpha$ - предельный ординал. Конфинальность $\alpha$ есть ординал $\operatorname{cf}(\alpha)$, равный наименьшему ординалу $\beta$, для которого существует такая функция $f$ из $\beta$ в $\alpha$, что

$$
\bigcup_{\zeta<\beta} f(\zeta)=\alpha .
$$

Кардинал $\kappa$ называется регулярным, если $\kappa=\operatorname{cf}(\kappa)$.

Так как $\operatorname{cf}(\alpha)=\operatorname{cf}(\operatorname{cf}(\alpha))$, то $\operatorname{cf}(\alpha)$ является регулярным кардиналом для любого ординала $\alpha$.

Следующие четыре утверждения доказываются просто. Доказательства последних двух приведены, например, в книге [17]. Доказательство первого приведено в книге [18]. Мы приводим здесь эти доказательства только ради полноты изложения. Второе утверждение мы доказываем здесь.

Теорема 2.1. Для каждого предельного ординала $\alpha>0$ имеет место равенство $\operatorname{cf}\left(\beth_{\alpha}\right)=\operatorname{cf}(\alpha)$.

ДокАзАтЕльство. Если существует такая функция $f$ из $\beta$ в $\alpha$, что

$$
\bigcup_{\zeta<\beta} f(\zeta)=\alpha,
$$

то, полагая $g(\gamma)=\beth_{f(\gamma)}$, мы получим, что

$$
\bigcup_{\zeta<\beta} g(\zeta)=\beth_{\alpha} .
$$

Обратно, если существует такая функция $g$ из $\beta$ в $\beth_{\alpha}$, что

$$
\bigcup_{\zeta<\beta} g(\zeta)=\beth_{\alpha},
$$

то, полагая $f(\gamma)$ равным наибольшему из таких $\zeta$, что $g(\gamma)$ содержит $\beth_{\zeta}$, мы получим, что

$$
\bigcup_{\zeta<\beta} f(\zeta)=\alpha .
$$

ТЕОрема 2.2. $\beth_{\alpha}^{*}=\beth_{\alpha}$ для любого предельного ординала $\alpha$.

ДокАЗАтЕльство. Если $\lambda<\beth_{\alpha}$, то $\lambda<\beth_{\beta}$ для некоторого $\beta<\alpha$. Поэтому $2^{\lambda} \leqslant 2^{\beth_{\beta}}=\beth_{\beta+1}$. Это доказывает, что $\beth_{\alpha}^{*} \leqslant \beth_{\alpha}$. Противоположное неравенство выполняется для всех кардиналов. 
Теорема 2.3 (Кёниг). Пусть $\kappa_{i}, \lambda_{i}-$ кардиналъ для всех $i \in I . \quad$ Если $\kappa_{i}<\lambda_{i}$ для всех $i \in I$, mo

$$
\sum_{i \in I} \kappa_{i}<\prod_{i \in I} \lambda_{i}
$$

ДокАзАтельство. Выберем для $i \in I$ множества $C_{i}$ мощности $\kappa_{i}$ и $T_{i}$ мощности $\lambda_{i}$. Будем предполагать, что $C_{i}$ является подмножеством $T_{i}$ и что $C_{i}$ и $C_{j}$ не имеют общих элементов для различных $i$ и $j$. Произвольный элемент $x$ из объединения множеств $C_{i}$ для $i \in I$ лежит в одном из объединяемых множеств, скажем, в $C_{j}$. Мы поставим этому $x$ в соответствие такой элемент $t_{x}$ декартова произведения $\prod_{i \in I} T_{i}$, что $t_{x}(j)=x$ и $t_{x}(i) \in\left(T_{i} \backslash C_{i}\right)$ для $i \in(I \backslash\{j\})$. Понятно, что $t_{x}$ и $t_{y}$ различны для различных $x$ и $y$. Это доказывает, что

$$
\sum_{i \in I} \kappa_{i} \leqslant \prod_{i \in I} \lambda_{i}
$$

Докажем теперь, что $\prod_{i \in I} T_{i}$ невозможно представить в виде объединения попарно не пересекающихся множеств $Z_{i}$ мощности $\kappa_{i}$ для $i \in I$. Допустим, напротив, что такое представление возможно. Пусть

$$
U_{i}=\left\{t(i) \mid t \in Z_{i}\right\}
$$

Ясно, что так построенное $U_{i}$ имеет мощность, не превосходящую $\kappa_{i}$. Возьмем такой элемент $f \in \prod_{i \in I} T_{i}$, что $f(i) \in\left(T_{i} \backslash U_{i}\right)$. Так построенный $f$ не лежит ни в одном $Z_{i}$. Полученное противоречие и доказывает теорему.

СлЕДСТвиЕ 2.4. $\operatorname{cf}\left(2^{\aleph_{\alpha}}\right)>\aleph_{\alpha}$.

ДокАзАТЕЛЬСтво. Надо доказать, что

$$
\sum_{\beta<\aleph_{\alpha}} \kappa_{\beta}<2^{\aleph_{\alpha}},
$$

если все эти $\kappa_{\beta}$ меньше $2^{\aleph_{\alpha}}$. Но по теореме Кёнига

$$
\sum_{\beta<\aleph_{\alpha}} \kappa_{\beta}<\prod_{\beta<\aleph_{\alpha}} 2^{\aleph_{\alpha}}=\left(2^{\aleph_{\alpha}}\right)^{\aleph_{\alpha}}=2^{\aleph_{\alpha}}
$$

ТЕОРема 2.5. Существуют кардиналь $\kappa=\kappa^{*}$ с как угодно большой конфинальностью.

ДокАЗАТЕЛЬСтво. Из предыдущих четырех утверждений следует, что, положив $\kappa=\beth_{2^{\aleph_{\alpha}}}$, мы получим, что $\kappa^{*}=\kappa$ и $\mathrm{cf}(\kappa)>\aleph_{\alpha}$. Это и означает, что существуют кардиналы $\kappa=\kappa^{*}$ с как угодно большой конфинальностью.

Нам понадобится следующая теорема.

Теорема 2.6 (Рамсея). Если множество всех $n$-элементных подмножеств бесконечного множества А разбито на $k$ частей, то в $A$ найдется бесконечное подмножество $B$, все $n$-элементные подмножества которого лежат в одной из этих частей. 
ДокАЗАтельство. Достаточно доказать теорему для $k=2$. Доказываем индукцией по $n$. При $n=1$ теорема тривиальна. Пусть она верна для $n$. Докажем ее для $n+1$.

Достаточно доказать теорему для счетного $A$. Занумеруем элементы $A$ натуральными числами. Если рассмотреть $(n+1)$-элементные подмножества $A$, содержащие первый элемент $a_{1}$ из $A$, и выбросить из каждого из них этот первый элемент, то полученные $n$-элементные подмножества будут разбиты на две части. По индукции, существует бесконечное подмножество $B_{2}$ множества $A \backslash\left\{a_{1}\right\}$, все $n$-элементные подмножества которого лежат в одной части. Если элементы $a_{1}, \ldots, a_{i}$ и не содержащее их бесконечное подмножество $B_{i+1}$ уже построены, пусть $a_{i+1}$ будет первым элементом в $B_{i+1}$. Если рассмотреть $(n+1)$-элементные подмножества множества $B_{i+1}$, содержащие $a_{i+1}$, и выбросить из каждого из них этот элемент, то полученные $n$-элементные подмножества будут разбиты на две части. По индукции, существует такое бесконечное подмножество $B_{i+2}$ множества $B_{i+1} \backslash\left\{a_{i+1}\right\}$, все $n$-элементные подмножества которого лежат в одной части. Так построенная последовательность попарно различных элементов $a_{1}, \ldots, a_{i}, \ldots$ обладает тем свойством, что все $(n+1)$-элементные подмножества, составленные из элементов этой последовательности, содержащие $a_{i}$ и не содержащие $a_{1}, \ldots, a_{i-1}$, лежат в одной и той же части $k(i)$. Осталось из последовательности $a_{1}, \ldots, a_{i}, \ldots$ выбрать такую подпоследовательность $a_{j_{1}}, \ldots, a_{j_{i}}, \ldots$, чтобы

$$
k\left(j_{1}\right)=\cdots=k\left(j_{i}\right)=\cdots .
$$

Теорема 2.6 доказана.

Нам понадобится также следующая теорема.

Теорема 2.7 (Рамсея о конечных множествах). Существует такое число $r(n, k, m)$, что для любых натуральных $n, k, m$ при любом разбиении всех $n$ элементных подмножеств конечного множества $A$, содержащего не менее

$$
r(n, k, m)
$$

элементов, на $k$ частей в $A$ найдется такое подмножество $B$, содержащее не менее $m$ элементов, все $n$-элементные подмножества которого лежат $в$ одной из этих частей.

ДокАЗАТЕЛЬство. Достаточно доказать теорему для $k=2$. За $r(1,2, m)$ можно взять $2 m$. Пусть $r(n, 2, m)$ уже определено. Вычислим $r(n+1,2, m)$. Пусть $s(n, m, 0)=2 r(n, 2, m)$ и $s(n, m, i+1)=r(n, 2, s(n, m, i))+1$.

Повторяя рассуждения из доказательства предыдущей теоремы Рамсея, легко видеть, что в качестве $r(n+1,2, m)$ можно взять $s(n, m, m+1)$.

\section{3. Предварительные сведения из теории моделей}

В этом разделе мы напоминаем некоторые начальные сведения из теории моделей. Даже если бы мы рассчитывали на читателя, знакомого с началами теории моделей, нам все равно пришлось бы договориться об обозначениях. Но 
мы имеем в виду также тех, кто интересуется теоретическим программированием, а они не обязательно изучали подробный курс математической логики.

Прежде всего, конечно, надо фиксировать предметную область, свойства которой мы намерены изучать. Эта область представляет собой непустое множество вместе с заданными на этом множестве операциями и отношениями. Эти заданные операции и отношения называются основными.

Чтобы иметь возможность записывать изучаемые свойства, потребуются обозначения или, другими словами, имена для заданных основных операций и отношений. Например, в арифметике предметная область - это множество $\omega$ натуральных чисел, основные операции - это безаргументные операции, выделяющие 0 и 1 , а также операции сложения и умножения, основное отношение это отношение порядка. В элементарной геометрии операций обычно не рассматривают, а основные отношения - это отношения равенства, принадлежности, быть точкой, быть прямой, быть плоскостью и другие.

Уже из этих примеров можно увидеть, что операции и отношения бывают от разного числа аргументов (как говорят, разной местности). Например, операции сложения и умножения двухместны (имеют два аргумента), а отношение быть точкой - от одного аргумента.

Oперация на множестве $A$ от $n$ аргументов представляет собой отображение, которое каждой последовательности длины $n$, составленной из элементов множества $A$, ставит в соответствие однозначно определенный элемент множества $A$. Например, операция сложения натуральных чисел имеет два аргумента и каждой паре натуральных чисел ставит в соответствие их сумму. Сумма определяется однозначно для каждой пары натуральных чисел, но различные пары могут иметь разные суммы.

Обозначим через $A^{n}$ множество всех последовательностей длины $n$, составленных из элементов множества $A$.

Каждое подмножество множества $A^{n}$ называется отношением или предикатом от $n$ аргументов на множестве $A$. Если последовательность $a_{1}, \ldots, a_{n}$ длины $n$, составленная из элементов множества $A$, входит в рассматриваемое отношение, то говорят, что это отношение $P$ истинно на этой последовательности, или что рассматриваемое отношение равно на этой последовательности 1 , или что $P\left(a_{1}, \ldots, a_{n}\right)$ истинно. Если последовательность $a_{1}, \ldots, a_{n}$ длины $n$, составленная из элементов множества $A$, не входит в рассматриваемое отношение $P$, то говорят, что это отношение ложно на этой последовательности, что рассматриваемое отношение равно на этой последовательности 0 и что $P\left(a_{1}, \ldots, a_{n}\right)$ ложно.

ОПРЕДЕЛЕНИЕ 1. Сигнатурой называется набор символов операций и отношений вместе с отображением, которое каждому символу приписывает натуральное число - число аргументных мест, или местность этого символа. Безаргументные символы операций называются сигнатурными константами или символами выделенных элементов. Мощность набора называется мощностъю сигнатуры. Сигнатурные символы иногда называются именами, соответственно, операций или отношений. 
ОПРЕДЕЛЕНИЕ 2. Алгебраической системой (иногда говорят также, струкmурой или интерпретацией) сигнатуры $L$ называется непустое множество вместе с отображением, которое каждому символу отношения из этой сигнатуры $L$ ставит в соответствие отношение той же местности на этом множестве, а каждому символу операции из сигнатуры $L$ ставит в соответствие операцию той же местности на этом же множестве. Это фиксированное непустое множество называется основным множеством рассматриваемой алгебраической системы или ее носителем. Мощность носителя называется мощностъю алгебраической системы.

Отношение, которое соответствует в алгебраической системе $A$ символу отношения $P$ из $L$, обозначается через $P^{A}$. Операция, которая соответствует в алгебраической системе $A$ символу операции $f$ из $L$, обозначается через $f^{A}$.

Приведем несколько примеров.

ПРимеР 1. Пусть основное множество является объединением следующих подмножеств:

1) фамилий преподавателей $\{$ Иванов, Петров, Сидоров, Степанов $\}$;

2) названий предметов \{алгебра, логика $\}$;

3) номеров аудиторий $\{201,202,203,204\}$;

4) дат в январе $\{3,4,5,6,7,8,9,10,11\}$;

5) шифров групп $\{M 1, M 2, M 3, M 4\}$.

Пусть сигнатура $L_{1}$ состоит из символов четырехместного отношения $R$ и трехместного отношения $P$.

Рассмотрим алгебраическую систему $A_{1}$ сигнатуры $L_{1}$ с указанным выше основным множеством, в которой $P$ и $R$ интерпретируются следующим образом. Отношение $P$ для каждой группы и каждого предмета указывает экзаменатора в этой группе по этому предмету и состоит из троек

$$
\begin{array}{ll}
\langle M 1, \text { алгебра, Иванов }\rangle, & \langle M 1, \text { логика, Сидоров }\rangle, \\
\langle M 2, \text { алгебра, Иванов }\rangle, & \langle M 2, \text { логика, Сидоров }\rangle, \\
\langle M 3, \text { алгебра, Петров }\rangle, & \langle M 3, \text { логика, Степанов }\rangle, \\
\langle M 4, \text { алгебра, Петров }\rangle, & \langle M 4, \text { логика, Степанов }\rangle .
\end{array}
$$

Отношение $R$ для каждой группы и предмета указывает дату и аудиторию экзамена по этому предмету в этой группе и состоит из четверок

$$
\begin{aligned}
& \langle M 1 \text {, алгебра, 3, 201〉, }\langle M 1 \text {, логика, 9, 201〉, }
\end{aligned}
$$

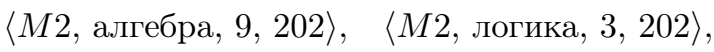

$$
\begin{aligned}
& \langle M 3 \text {, алгебра, 5, 203〉, }\langle M 3 \text {, логика, 11, 204〉, } \\
& \langle M 4 \text {, алгебра, 9, 203〉, }\langle M 4 \text {, логика, 3, 203〉. }
\end{aligned}
$$

Алгебраическая система $A_{1}$ описывает расписание экзаменов в рассматриваемых группах.

ПримеР 2. Расписание экзаменов в тех же группах можно задать и подругому, рассматривая сигнатуру $L_{2}$, состоящую из символа пятиместного отношения $Q$, и такую алгебраическую систему $A_{2}$ сигнатуры $L_{2}$, что основное 
множество системы $A_{2}$ совпадает с основным множеством системы $A_{1}$, а отношение $Q$ в $A_{2}$ состоит из пятерок:

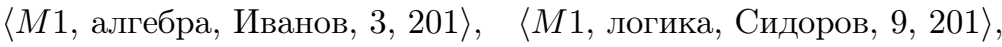
$\langle M 2$, алгебра, Иванов, 9, 202 $\rangle, \quad\langle M 2$, логика, Сидоров, 3,202$\rangle$,
$\langle M 3$, алгебра, Петров, 5, 203〉, $\langle M 3$, логика, Степанов, 11, 204〉,
$\langle M 4$, алгебра, Петров, 9, 203〉, $\langle M 4$, логика, Степанов, 3, 203〉.

В пятерке идут шифр группы, название предмета, фамилия преподавателя, дата экзамена, аудитория.

Подмножество носителя алгебраической системы замкнуто относителъно операции, определенной на основном множестве этой системы, если из того, что какие-то аргументы лежат в этом подмножестве, следует, что и значение рассматриваемой операции на этом наборе аргументов лежит в этом подмножестве. В частности, для константы это означает, что рассматриваемое подмножество содержит значение этой константы. Ограничением операции $f \mathrm{c}$ числом аргументов $m$ на замкнутое относительно $f$ множество $D$ называется определенная на $D$ операция $g$, которая имеет столько же аргументов, сколько имеет $f$, и для которой $g\left(a_{1}, \ldots, a_{m}\right)=f\left(a_{1}, \ldots, a_{m}\right)$ для любых $a_{1}, \ldots, a_{m}$ из $D$. Ограничением $m$-местного отношения $P$ на $D$ называется $P \cap D^{m}$.

Операции, соответствующие сигнатурным символам операций в рассматриваемой алгебраической системе, называются основными операциями этой алгебраической системы. При этом элементы, соответствующие сигнатурным константам, называются также выделенными элементами этой алгебраической системы.

Подмножество носителя алгебраической системы $A$ сигнатуры $L$, замкнутое относительно всех основных операций этой алгебраической системы, вместе с ограничениями всех основных операций и отношений на это подмножество образует алгебраическую систему сигнатуры $L$, которая называется подсистемой системы $A$. Запись $A \subseteq B$ означает, что $A$ является подсистемой системы $B$. Если $A$ является подсистемой системы $B$, то $B$ называется расширением системы $A$.

Сейчас мы приступаем к определению логики предикатов первого порядка.

Обозначения для суперпозиций основных операций обычно называют термами.

В термах используются предметные переменные - переменные, принимающие значения из основного множества рассматриваемой алгебраической системы. Мы будем обозначать предметные переменные строчными латинскими буквами $x, y, z$ с индексами.

ОпрЕДЕлЕниЕ 3. а) Каждая предметная переменная и каждый нульместный символ операции сигнатуры $L$ - терм сигнатуры $L$. В предметную переменную входит она сама, а другие переменные не входят. В символ операции предметные переменные не входят.

b) Если $f-n$-местный символ операции сигнатуры $L$, а $t_{1}, \ldots, t_{n}$ являются термами сигнатуры $L$, то $f\left(t_{1}, \ldots, t_{n}\right)$ - тоже терм сигнатуры $L$. Переменная 
входит в $f\left(t_{1}, \ldots, t_{n}\right)$ тогда и только тогда, когда она входит хотя бы в один из термов $t_{1}, \ldots, t_{n}$.

c) Каждое выражение является термом сигнатуры $L$, только если это можно доказать, используя пункты а) и b).

Терм, не содержащий переменных, называется замкнутым.

Обычно при двухместном $f$ вместо $f\left(t_{1}, t_{2}\right)$ пишут $\left(t_{1} f t_{2}\right)$. Например, пишут $\left(x_{1}+x_{2}\right)$ вместо $+\left(x_{1}, x_{2}\right)$.

В частности, $\left(\left(x_{1}+x_{2}\right) \times x_{3}\right)$ - арифметический терм, в котором + и $\times-$ символы двухместных операций сложения и умножения.

Заметим, что если сигнатура не содержит символов операций, то каждый терм является просто предметной переменной, так как в этом случае пункт b) определения нельзя использовать.

ОПРЕДЕЛЕНИЕ 4. Состоянием памяти (или оценкой) алгебраической системы называется отображение, которое каждой предметной переменной ставит в соответствие некоторый элемент основного множества этой системы.

ОПРЕДЕЛЕниЕ 5. Пусть $\sigma$ - состояние алгебраической системы $A$ сигнатуры $L$.

a) Если $x$ - предметная переменная, то $\sigma(x)$ задано по определению $\sigma$.

b) Если $f$ - нульместный символ операции сигнатуры $L, f^{A}$ - безаргументная операция, поставленная в соответствие символу $f$ в алгебраической системе $A$, то $\sigma(f)$ есть значение $f^{A}$.

c) Если $f-n$-местный $(n>0)$ символ операции сигнатуры $L, f^{A}$ - операция, соответствующая символу $f$ в алгебраической системе $A$, a $t_{1}, \ldots, t_{n}$ - термы сигнатуры $L$, то

$$
\sigma\left(f\left(t_{1}, \ldots, t_{n}\right)\right)
$$

есть

$$
f^{A}\left(\sigma\left(t_{1}\right), \ldots, \sigma\left(t_{n}\right)\right)
$$

Элемент $\sigma(t)$ называется значением терма $t$ на состоянии $\sigma$.

Например, если $\sigma\left(x_{1}\right)=1, \sigma\left(x_{2}\right)=2, \sigma\left(x_{3}\right)=7$, то $\sigma\left(\left(\left(x_{1}+x_{2}\right) \times x_{3}\right)\right)=21$ при стандартной интерпретации символов + и $\times$.

Для записи свойств элементов алгебраической системы используют формулы.

ОПРЕДЕЛЕНИЕ 6 . а) Если $t_{1}, t_{2}$ - термы сигнатуры $L$, то $t_{1}=t_{2}$ - атомная формула сигнатуры $L$. В $t_{1}=t_{2}$ входят те и только те переменные, которые входят в $t_{1}$ или в $t_{2}$.

b) Если $P$ - нульместный символ отношения сигнатуры $L$, то $P$ - атомная формула сигнатуры $L$, в которую никакие предметные переменные не входят.

c) Если $P-n$-местный $(n>0)$ символ отношения сигнатуры $L$, а $t_{1}, \ldots, t_{n}-$ термы сигнатуры $L$, то

$$
P\left(t_{1}, \ldots, t_{n}\right)
$$

является атомной формулой сигнатуры $L$. В эту формулу входят те и только те переменные, которые входят хотя бы в один из термов $t_{1}, \ldots, t_{n}$. 
При двухместном $P$ иногда вместо $P\left(t_{1}, t_{2}\right)$ пишут $t_{1} P t_{2}$ или $\left(t_{1} P t_{2}\right)$, например, вместо $<\left(t_{1}, t_{2}\right)$ пишут $t_{1}<t_{2}$.

ОПРЕдЕлЕниЕ 7 (формулы логики предикатов). а) Атомная формула сигнатуры $L$ является формулой сигнатуры $L$. Все переменные, входящие в атомную формулу, входят в нее свободно и не входят связанно.

b) Если $\Phi, \Psi$ - формулы сигнатуры $L$, то $\neg \Phi,(\Phi \vee \Psi),(\Phi \wedge \Psi),(\Phi \rightarrow \Psi)$ - тоже формулы сигнатуры $L$. Переменная входит свободно (связанно) в $\neg \Phi$ тогда и только тогда, когда эта переменная входит свободно (соответственно связанно) в $\Phi$. Переменная входит свободно (связанно) в $(\Phi \vee \Psi),(\Phi \wedge \Psi),(\Phi \rightarrow \Psi)$ тогда и только тогда, когда эта переменная входит свободно (соответственно связанно) хотя бы в одну из формул $\Phi$ или $\Psi$ или в обе эти формулы.

c) Если $\Phi$ - формула сигнатуры $L, x$ - переменная, то $(\forall x) \Phi$ и $(\exists x) \Phi$ - тоже формулы сигнатуры $L$. Переменная $x$ входит в обе эти формулы связанно и не входит свободно. Отличная от $x$ переменная входит в $(\forall x) \Phi,(\exists x) \Phi$ свободно (связанно) тогда и только тогда, когда эта переменная входит свободно (соответственно связанно) в $\Phi$.

d) Каждое выражение является формулой сигнатуры $L$, только если это можно доказать, используя a), b) и с).

Формула, в которую никакая переменная не входит свободно, называется замкнутой.

Символы $\wedge, \vee, \rightarrow, \neg$ обычно называют (пропозициональными) связками, а $\forall$ и $\exists$ - знаками кванторов. При этом $\forall$ - знак квантора общности, а $\exists-$ знак квантора существования. Если $x$ - переменная, то выражения $(\forall x),(\exists x)-$ кванторы общности и существования соответственно по переменной $x$.

Формулы $(\forall x) \Phi$ и $(\exists x) \Phi$ получаются из $\Phi$ навешиванием квантора.

В формулах $(\forall x) \Phi$ и $(\exists x) \Phi$ формула $\Phi$ называется областью действия квантора $(\forall x)$ или $(\exists x)$. Грубо говоря, связанное вхождение переменной - это вхождение в область действия квантора по той же переменной.

Приведем несколько примеров.

ПримеР 3. Рассмотрим сигнатуру $L_{1}$, описанную в примере 1 . Выражение

$$
\begin{gathered}
(\forall z)(\forall x)(\forall y)\left(\left(\left(\exists x_{1}\right)\left(\exists y_{1}\right) R\left(x, z, x_{1}, y_{1}\right) \wedge(\exists z)\left(\exists x_{2}\right)\left(\exists y_{2}\right) R\left(y, z, x_{2}, y_{2}\right)\right)\right. \\
\left.\rightarrow\left(\exists x_{2}\right)\left(\exists y_{2}\right) R\left(y, z, x_{2}, y_{2}\right)\right)
\end{gathered}
$$

является формулой сигнатуры $L_{1}$, содержащей связанно $x, y, z, x_{1}, y_{1}, x_{2}, y_{2}$ и не содержащей свободных переменных.

ПримеР 4. Рассмотрим сигнатуру $L_{2}$, описанную в примере 2 . Выражение

$$
\left(\left(\exists x_{1}\right)\left(\exists y_{1}\right)\left(\exists z_{1}\right) Q\left(x_{3}, x_{1}, y, y_{1}, z_{1}\right) \wedge\left(\exists x_{2}\right)\left(\exists y_{2}\right)\left(\exists z_{2}\right) Q\left(x_{1}, x_{2}, y, y_{2}, z_{2}\right)\right)
$$

является формулой сигнатуры $L_{2}$, в которую $x_{1}, y_{1}, z_{1}, x_{2}, y_{2}, z_{2}$ входят связанно, а $x_{3}, y, x_{1}$ входят свободно. Из этого примера видно, что одна и та же переменная может входить в рассматриваемую формулу и свободно, и связанно. 


\begin{tabular}{|c|c|}
\multicolumn{2}{|c|}{ ТАБлицА 1} \\
\hline$\sigma\left(\Phi_{1}\right)$ & $\sigma\left(\neg \Phi_{1}\right)$ \\
\hline 0 & 1 \\
\hline 1 & 0 \\
\hline
\end{tabular}

ТАБЛИцА 2

\begin{tabular}{|c|c|c|c|c|}
\hline$\sigma\left(\Phi_{1}\right)$ & $\sigma\left(\Phi_{2}\right)$ & $\sigma\left(\Phi_{1} \wedge \Phi_{2}\right)$ & $\sigma\left(\Phi_{1} \vee \Phi_{2}\right)$ & $\sigma\left(\Phi_{1} \rightarrow \Phi_{2}\right)$ \\
\hline \hline 0 & 0 & 0 & 0 & 1 \\
\hline 0 & 1 & 0 & 1 & 1 \\
\hline 1 & 0 & 0 & 1 & 0 \\
\hline 1 & 1 & 1 & 1 & 1 \\
\hline
\end{tabular}

ОПредЕЛЕНИЕ 8 (значения формулы). Пусть $A$ - алгебраическая система сигнатуры $L, \Phi$ - формула сигнатуры $L, \sigma$ - состояние системы $A$.

a) Если $\Phi$ есть $t_{1}=t_{2}$, где $t_{1}$ и $t_{2}$ - термы сигнатуры $L$, то $\sigma(\Phi)=1$, если

$$
\sigma\left(t_{1}\right)=\sigma\left(t_{2}\right)
$$

и $\sigma(\Phi)=0$, если

$$
\sigma\left(t_{1}\right) \neq \sigma\left(t_{2}\right)
$$

b) Пусть $\Phi$ есть $P, P$ - нульместный символ отношения из сигнатуры $L$ и $P^{A}$ - отношение, поставленное в соответствие символу $P$ в системе $A$. Тогда $\sigma(P)$ есть $P^{A}$.

c) Пусть $\Phi$ есть $P\left(t_{1}, \ldots, t_{n}\right)$, где $P-n$-местный символ отношения из сигнатуры $L$, а $t_{1}, \ldots, t_{n}$ - термы сигнатуры $L, P^{A}$ - отношение, поставленное в соответствие символу $P$ в системе $A$. Тогда $\sigma\left(P\left(t_{1}, \ldots, t_{n}\right)\right)=1$, если

$$
\left\langle\sigma\left(t_{1}\right), \ldots, \sigma\left(t_{n}\right)\right\rangle \in P^{A},
$$

и $\sigma\left(P\left(t_{1}, \ldots, t_{n}\right)\right)=0$, если

$$
\left\langle\sigma\left(t_{1}\right), \ldots, \sigma\left(t_{n}\right)\right\rangle \notin P^{A}
$$

d) Если $\Phi$ есть одна из формул $\neg \Phi_{1},\left(\Phi_{1} \vee \Phi_{2}\right),\left(\Phi_{1} \wedge \Phi_{2}\right),\left(\Phi_{1} \rightarrow \Phi_{2}\right)$, то $\sigma(\Phi)$ определяется через $\sigma\left(\Phi_{1}\right)$ и $\sigma\left(\Phi_{2}\right)$ по правилам, указанным в таблицах 1 и 2 .

е) Если $\Phi$ есть $(\exists x) \Phi_{1}$, то $\sigma(\Phi)=1$ тогда и только тогда, когда существует такое состояние $\sigma_{1}$ системы $A$, что $\sigma_{1}\left(\Phi_{1}\right)=1$ и $\sigma_{1}(y)=\sigma(y)$ для каждой отличной от $x$ переменной $y$.

f) Если $\Phi$ есть $(\forall x) \Phi_{1}$, то $\sigma(\Phi)=1$ тогда и только тогда, когда $\sigma_{1}\left(\Phi_{1}\right)=1$ для каждого такого состояния $\sigma_{1}$ системы $A$, что $\sigma_{1}(y)=\sigma(y)$ для каждой отличной от $x$ переменной $y$.

Если $\sigma(\Phi)=1$, то говорят, что $\Phi$ истинна на $\sigma$ и пишут $\sigma \models \Phi$. Если $\sigma(\Phi)=0$, то говорят, что $\Phi$ ложна на $\sigma$. Если $\Phi$ истинна на каждом состоянии системы $A$, то говорят, что $\Phi$ истинна в $A$ (или на $A$ ), а также, что $A$ является моделъю $\Phi$ или для $\Phi$, и пишут $A \models \Phi$. 
Если $\Phi$ истинна в каждой алгебраической системе сигнатуры $L$, то говорят, что $\Phi$ тождественно истинна.

Легко заметить, что

$$
\begin{aligned}
& \sigma((\forall x) \Phi)=\sigma(\neg(\exists x) \neg \Phi), \\
& \sigma((\exists x) \Phi)=\sigma(\neg(\forall x) \neg \Phi)
\end{aligned}
$$

для каждой формулы $\Phi$ и каждого состояния $\sigma$.

Формулы $\Phi$ и $\Psi$ называются эквивалентными в алгебраической системе $A$, если $\sigma(\Phi)=\sigma(\Psi)$ для каждого состояния $\sigma$ в $A$. Формулы $\Phi$ и $\Psi$ называются эквивалентными, если они эквивалентны в каждой алгебраической системе рассматриваемой сигнатуры. Запись $\Phi \equiv \Psi$ означает, что $\Phi$ и $\Psi$ эквивалентны.

Формула, которая не содержит кванторов, называется бескванторной. Формула называется предваренной, если она бескванторная или имеет вид $(\forall x) \Phi$ или вид $(\exists x) \Phi$, где $\Phi$ является предваренной формулой, а $x$ - переменной. Таким образом, в предваренной формуле сначала идут кванторы, а потом идет бескванторная формула. Другими словами, предваренная формула получается из бескванторной навешиванием кванторов. В предваренной формуле ее начало, состоящее из кванторов и стоящее перед бескванторной формулой, называется кванторной приставкой.

Легко доказываются следующие две теоремы.

Теорема 3.1. Каждая формула эквивалентна некоторой предваренной формуле.

ТеОрема 3.2. Пусть все свободные переменные бормулы $\Phi$ сигнатуры $L$ содержатся среди $x_{1}, \ldots, x_{m}$. Пусть $\sigma_{1}$ u $\sigma_{2}$ - состояния алгебраической системы А сигнатуры L. Если

$$
\sigma_{1}\left(x_{i}\right)=\sigma_{2}\left(x_{i}\right)
$$

для $i=1, \ldots, m, \operatorname{mo} \sigma_{1}(\Phi)=\sigma_{2}(\Phi)$.

Из теоремы 3.2 следует, что истинность замкнутой формулы не зависит от состояния алгебраической системы, а зависит только от самой алгебраической системы.

Рассмотрим, например, формулу (1) и алгебраическую систему $A_{1}$, описанную в примере 1 . Как эта формула, так и система $A_{1}$ имеют сигнатуру $L_{1}$, описанную в том же примере. Формула $(1)$ имеет вид $(\forall z)(\forall x)(\forall y) \Phi$. Формула (1) истинна в $A_{1}$.

Формулы сигнатуры $L$ называются $L$-формулами. Алгебраическая система сигнатуры $L$ называется также $L$-системой или $L$-структурой. Для $L$-формулы $\Phi$ и кортежа переменных $\bar{x}$ запись $\Phi(\bar{x})$ означает, что формула $\Phi$ не содержит свободных переменных, не входящих в кортеж $\bar{x}$. Если $\bar{a}-$ кортеж той же длины, что и кортеж $\bar{x}$, элементов некоторой $L$-структуры, то, ради краткости, через $\Phi(\bar{a})$ обозначается значение $\Phi(\bar{x})$ на любом таком состоянии этой $L$-структуры, которое переменным из кортежа $\bar{x}$ приписывает соответственно значения из кортежа $\bar{a}$. 
$L$-теорией называется любое множество замкнутых $L$-формул. $L$-теория называется совместной, если существует алгебраическая система сигнатуры $L$, на которой все формулы из этой $L$-теории истинны. Эта алгебраическая система называется моделъю рассматриваемой $L$-теории. $L$-теория называется конечно совместной, если каждое ее конечное подмножество совместно.

Элементарной теорией или $L$-теорией класса $K$ (обозначается $\operatorname{Th}(K)$ ), состоящего из $L$-структур, называется совокупность всех замкнутых $L$-формул, истинных во всех системах из класса $K$. Если класс состоит из одной $L$-структуры $A$, то его элементарная теория называется элементарной теорией или $L$-теорией этой $L$-структуры $A$ и обозначается через $\operatorname{Th}(A)$.

Две $L$-структуры называются элементарно эквивалентными, если каждая замкнутая $L$-формула, истинная в одной из этих алгебраических систем, истинна и в другой. В этом случае элементарные теории этих $L$-структур совпадают. $L$-теория называется nолной, если любые две ее модели элементарно эквивалентны. Запись $A \equiv B$ означает, что $L$-структуры $A$ и $B$ элементарно эквивалентны.

Пусть сигнатура $L^{\prime}$ является частью сигнатуры $L$. Это означает, что каждый символ операции из $L^{\prime}$ входит в $L$ как символ операции и имеет в $L$ то же число аргументных мест, что и в $L^{\prime}$, а каждый символ отношения из $L^{\prime}$ входит в $L$ как символ отношения и имеет в $L$ то же число аргументных мест, что и в $L^{\prime}$. В этом случае $L^{\prime}$ называется обеднением $L$, а $L$ называется обогащением $L^{\prime}$.

Пусть $L^{\prime}$ является обеднением сигнатуры $L$. Тогда из каждой $L$-структуры $A$ можно получить $L^{\prime}$-структуру, отбрасывая лишние операции и отношения. Эта полученная $L^{\prime}$-структура $B$ обозначается как $A \uparrow L^{\prime}$ и называется $L^{\prime}$-обеднением $L$-структуры $A$. Структура $A$ называется при этом $L$-обогащением $L^{\prime}$-структуры $B$.

Для $L$-структуры $M$ и произвольного подмножества $A$ носителя структуры $M$ через $L(A)$ обозначают сигнатуру, получаемую добавлением к $L$ имен для всех элементов $A$. Эти имена являются символами выделенных элементов. Обычно мы не различаем элемент и его имя. Если $A$ совпадает с носителем $L$-структуры $M$, то вместо $L(A)$ мы пишем $L(M)$. Через $(M, a \mid a \in A)$ обозначается такое обогащение $L$-структуры $M$ до $L(A)$-структуры, в котором значением добавленного имени элемента является сам этот элемент.

Иногда, ради краткости, мы будем говорить об истинности некоторой замкнутой $L(A)$-формулы в $L$-структуре $M$, понимая под этим истинность этой формулы в $(M, a \mid a \in A)$.

$L$-структура $M$ называется элементарной подсистемой $L$-структуры $N$, а $L$-структура $N$ называется элементарным расширением $L$-структуры $M$, если $M$ является подсистемой системы $N$ и любая замкнутая $L(M)$-формула $\phi$ тогда и только тогда истинна в $(M, a|a \in| M \mid)$, когда она истинна в $(N, a|a \in| M \mid)$. Здесь $|M|$ обозначает носитель системы $M$. Записи $M \preceq N$ и $N \succeq M$ означают, что $M$ является элементарной подсистемой системы $N$.

Рассмотрим последовательность $L$-структур $A_{\alpha}, \alpha \in I$, где $I$ - упорядоченное множество. Эта последовательность называется возрастающей, если $A_{\alpha} \subseteq A_{\beta}$ для любых $\alpha<\beta$ из $I$. Объединением возрастающей последовательности подсистем $A_{\alpha}(\alpha \in I)$ называется такая $L$-структура $B$, носитель которой 
является объединением носителей структур $A_{\alpha}(\alpha \in I)$ и которая является расширением каждой из систем $A_{\alpha}(\alpha \in I)$.

Легко доказывается следующая теорема.

ТЕОрема 3.3. Обгединение возрастающей последователъности элементарных подсистем является элементарным расширением каждой из этих подсистем.

Главными теоремами современной теории моделей являются следующие две теоремы.

ТЕОрема 3.4 (Лёвенгейм, Скулем). Пусть мощность сигнатуры L не превосходит бесконечного кардинала $\kappa$. Для каждой бесконечной $L$-структуры $N$ и каждого подмножества $А$ мощности к носителя структуры $N$ существует элементарная подсистема $M$ системы $N$, носитель которой содержит $A$ и имеет мощность $\kappa$.

ТеОрема 3.5 (компактности Мальцева). Каждая конечно совместная Lтеория совместна.

Доказательства этих теорем можно найти, например, в [18].

Очевидным следствием теоремы компактности Мальцева является следующая

ТеОРема 3.6 (о расширении). Каждая бесконечная алгебраическая система имеет элементарное расширение как угодно большой мощности.

Для $L$-структуры $M$ и произвольного подмножества $A$ носителя структуры $M$ конечное множество $\left\{\phi_{1}(x), \ldots, \phi_{k}(x)\right\}$, состоящее из $L(A)$-формул, не содержащих свободных переменных, отличных от $x$, выполняется в $(M, a \mid a \in A)$, если формула

$$
(\exists x)\left(\phi_{1}(x) \wedge \cdots \wedge \phi_{k}(x)\right)
$$

истинна в $M$.

Для $L$-структуры $M$ и произвольного подмножества $A$ носителя структуры $M$ множество $p$, состоящее из $L(A)$-формул, не содержащих свободных переменных, отличных от $x$, называется конечно выполнимым над $A$ в $M$, если каждое конечное подмножество $\left\{\phi_{1}(x), \ldots, \phi_{k}(x)\right\}$ множества $p$ выполняется в $(M, a \mid a \in A)$.

Конечно выполнимое множество $p$ называется типом над $A$ в $M$, если для каждой $L(A)$-формулы $\phi(x)$ либо $\phi \in p$, либо $\neg \phi \in p$.

Говорят, что подмножество $q$ типа $p$ изолирует $p$, если $p$ есть единственный тип над $A$ в $M$, содержащий $q$.

Рассмотрим $L$-структуру $M$ и произвольное подмножество $A$ носителя структуры $M$.

Для любых $N \succeq M$ и $b \in N$ множество всех таких $L(A)$-формул $\phi(x)$, что $\phi(b)$ истинно в

$$
(N, a \mid a \in A),
$$

образует тип над $A$ в $M$. Обозначим этот тип через $\operatorname{tp}(b / A)$. Будем говорить, что $b \in N$ реализует тип $\operatorname{tp}(b / A)$ и что этот тип реализуется в $N$. 
Очевидным следствием теоремы компактности Мальцева является следующая теорема.

Теорема 3.7. Рассмотрим L-структуру $M$ и произволъное подмножество А носителя структуры $M$. Для любого типа р над А в $M$ существует такое $N \succeq M$, что р реализуется в $N$. Другими словами, существует такой $a \in N$, что $p=\operatorname{tp}(a / A)$.

Рассмотрим некоторый бесконечный кардинал $\lambda$.

ОПРЕДЕЛЕНИЕ 9 ( $\lambda$-насыщенной структуры). $L$-структура $M$ называется $\lambda$ насыщенной, если для каждого подмножества $A$ мощности меньше $\lambda$ носителя этой структуры и каждого типа $p$ над $A$ этот тип $p$ реализуется в $M$.

Это означает, что для каждого подмножества $A$ мощности меньше $\lambda$ носителя этой структуры $M$ и каждого типа $p$ в $M$ над $A$ найдется такой элемент $a \in M$, что $p=\operatorname{tp}(a / A)$ в $M$.

Легко понять, что каждая конечная $L$-структура является $\lambda$-насыщенной для каждого бесконечного кардинала $\lambda$.

Каждая $\lambda$-насыщенная $L$-структура мощности $\lambda$ называется насыщенной.

ОПРЕДЕЛЕНИЕ 10 (частичного изоморфизма $A$ в $B$ ). Пусть $A$ и $B$ - алгебраические системы сигнатуры $L$. Пусть $C$ - подмножество носителя системы $A$ и $D$ - подмножество носителя системы $B$. Пусть $\tau$ одно-однозначно отображает $C$ на $D$. Это означает, что $\tau$ есть некоторое множество пар вида $(a, b)$, где $a \in C$ и $b \in D, \tau$ содержит одну и только одну пару вида $(a, b)$ для каждого $a \in C$ и $\tau$ содержит одну и только одну пару вида $(a, b)$ для каждого $b \in D$. Если $(a, b) \in \tau$, то $\tau(a)$ есть $b$ и $\tau^{-1}(b)$ есть $a$.

Это отображение $\tau$ называется частичным изоморфизмом из $A$ в $B$, если

1) для каждого символа отношения $P$ из $L$ и каждых $d_{1}, \ldots, d_{k}$ из $C$ выполняется $P^{A}\left(d_{1}, \ldots, d_{k}\right)$ тогда и только тогда, когда выполняется $P^{B}\left(\tau\left(d_{1}\right)\right.$, $\ldots, \tau\left(d_{k}\right)$ ), где $k$ есть число аргументных мест $P$ (при $k=0$ это означает, что $P^{A}$ совпадает с $\left.P^{B}\right)$;

$2)$ для каждого ненульместного символа операции $f$ из $L$ с числом $k$ аргументных мест и каждых $d_{1}, \ldots, d_{k}$ из $C$ тогда и только тогда $f^{A}\left(d_{1}, \ldots, d_{k}\right)$ лежит в $C$, когда $f^{B}\left(\tau\left(d_{1}\right), \ldots, \tau\left(d_{k}\right)\right)$ лежит в $D$, и

$$
\tau\left(f^{A}\left(d_{1}, \ldots, d_{k}\right)\right)=f^{B}\left(\tau\left(d_{1}\right), \ldots, \tau\left(d_{k}\right)\right),
$$

если $f^{A}\left(d_{1}, \ldots, d_{k}\right)$ лежит в $C$;

3) для каждого символа выделенного элемента $c$ из $L$

(a) если $c^{A} \in C$, то $c^{B} \in D$ и $\tau\left(c^{A}\right)=c^{B}$;

(b) если $c^{B} \in D$, то $c^{A} \in C$ и $\tau^{-1}\left(c^{B}\right)=c^{A}$.

Частичный изоморфизм $\tau$ называется изоморфизмом между $A$ и $B$, если $A=C$ и $B=D$. Изоморфизм между $A$ и $A$ называется автоморфизмом. Две структуры одной сигнатуры называются изоморфными, если существует изоморфизм между ними.

Легко заметить, что любые две конечные $L$-структуры тогда и только тогда элементарно эквивалентны, когда они изоморфны. 
ТЕОРема 3.8. Для любого бесконечного $\lambda$ любые две элементарно эквивалентные $\lambda$-насыщенные $L$-структуры $A$ и $B$ мощности $\lambda$ изоморфны.

ДокАЗАТЕЛЬСтво. Пусть $|A|=\left\{a_{\alpha} \mid \alpha<\lambda\right\}$ и $|B|=\left\{b_{\alpha} \mid \alpha<\lambda\right\}$.

Для $\alpha<\lambda$ индукцией по $\alpha$ будем строить такие отображение $\tau_{\alpha}$ и множества $C_{\alpha}$ и $D_{\alpha}$, что

(a) $\tau_{\alpha}$ одно-однозначно отображает $C_{\alpha}$ на $D_{\alpha}$;

(b) $\left\{a_{\beta} \mid \beta<\alpha\right\} \subseteq C_{\alpha} \subseteq|A|$;

(c) $\left\{b_{\beta} \mid \beta<\alpha\right\} \subseteq D_{\alpha} \subseteq|B|$;

(d) $\left(A, d \mid d \in C_{\alpha}\right) \equiv\left(B, \tau_{\alpha}(d) \mid d \in C_{\alpha}\right)$.

Здесь $\left(A, d \mid d \in C_{\alpha}\right)$ и $\left(B, \tau_{\alpha}(d) \mid d \in C_{\alpha}\right)$ являются $L\left(C_{\alpha}\right)$-структурами. $\mathrm{B}\left(A, d \mid d \in C_{\alpha}\right)$ значением имени элемента из $C_{\alpha}$ является сам этот элемент, а в $\left(B, \tau_{\alpha}(d) \mid d \in C_{\alpha}\right)$ значением имени элемента $d$ из $C_{\alpha}$ является $\tau_{\alpha}(d)$.

Мы полагаем $C_{0}$ и $D_{0}$ пустыми. Для предельных $\alpha$ пусть $\tau_{\alpha}$ является объединением всех $\tau_{\beta}$ для $\beta<\alpha$. Считаем, что $\tau_{\alpha}$ уже построено, $\alpha<\lambda$, и строим $\tau_{\alpha+1}$.

Если $a_{\alpha}$ содержится в $C_{\alpha}$, то пусть $\tau_{\alpha}^{\prime}$ совпадает с $\tau_{\alpha}$ и $b=\tau_{\alpha}\left(a_{\alpha}\right)$. Иначе рассмотрим $p=\operatorname{tp}\left(a_{\alpha} / C_{\alpha}\right)$. Из (d) следует, что $p$ является типом над $D_{\alpha}$ в $B$, если значением имени элемента $d$ из $C_{\alpha}$ является $\tau_{\alpha}(d)$. Из $\lambda$-насыщенности $B$ следует, что этот тип реализуется некоторым элементом $b$. Мы полагаем $\tau_{\alpha}^{\prime}\left(a_{\alpha}\right)=b$ и $\tau_{\alpha}^{\prime}(a)=\tau_{\alpha}(a)$ для $a \in C_{\alpha}$. Таким образом, $\tau_{\alpha}^{\prime}$ одно-однозначно отображает $C_{\alpha} \cup\left\{a_{\alpha}\right\}$ на $D_{\alpha} \cup\{b\}$. Если $b_{\alpha}$ содержится в $D_{\alpha} \cup\{b\}$, то $\tau_{\alpha+1}$ совпадает с $\tau_{\alpha}^{\prime}$. Иначе рассмотрим тип $b_{\alpha}$ над $D_{\alpha} \cup\{b\}$. Обозначим его через $p^{\prime}$. Из (d) и выбора $b$ следует, что $p^{\prime}$ является типом над $C_{\alpha} \cup\left\{a_{\alpha}\right\}$ в $A$, если значением имени элемента $d$ из $D_{\alpha}$ является $\tau_{\alpha}^{-1}(d)$, а значением имени элемента $b$ является $a_{\alpha}$. Из $\lambda$-насыщенности $A$ следует, что этот тип реализуется в $A$ некоторым элементом $a$. Мы полагаем $\tau_{\alpha+1}(a)=b_{\alpha}$ и $\tau_{\alpha+1}(d)=\tau_{\alpha}^{\prime}(d)$ для $d \in\left(C_{\alpha} \cup\left\{a_{\alpha}\right\}\right)$. Легко проверить, что условия (a)-(d) для так построенного $\tau_{\alpha+1}$ выполнены.

Из (a), (b) и (c) получаем, что $\tau_{\lambda}$ одно-однозначно отображает $|A|$ на $|B|$. Из $(\mathrm{d})$ легко следует, что это отображение является изоморфизмом между $A$ и $B$.

ТЕОРема 3.9 (о существовании $\lambda$-насыщенных структур). Пусть мощность сигнатуры $L$ не превосходит $\lambda$. Пусть бесконечная L-структура A имеет мощность, не превосходящую $2^{\lambda}$. Тогда существует такое $\lambda^{+}$-насыщенное элементарное расширение $B$ системы $A$, которое имеет мощность $2^{\lambda}$.

ДокАЗАТЕЛЬСТво. Построим такую возрастающую последовательность элементарных подсистем $B_{\alpha}$ длины $2^{\lambda}$, что

(a) $B_{0}$ есть $A$;

(b) для любого подмножества $X$ мощности $\lambda$ носителя $B_{\alpha}$ каждый тип над $X$ в $B_{\alpha}$ реализуется в $B_{\alpha+1}$;

(с) для $\alpha>0$ мощность $B_{\alpha}$ равна $2^{\lambda}$.

Для построения $B_{\alpha+1}$ найдем такую $L$-структуру $B$, в которой реализуются все типы над всеми подмножествами $X$ мощности $\lambda$ носителя $B_{\alpha}$ и которая является элементарным расширением $B_{\alpha}$. Существование такой $B$ легко следует 
из теоремы компактности Мальцева. По теореме о расширении, можно считать, что мощность $B$ не меньше $2^{\lambda}$. Так как таких типов не больше, чем $2^{\lambda}$, то, по теореме Лёвенгейма-Скулема, в $B$ имеется элементарная подсистема $B_{\alpha+1}$ мощности $2^{\lambda}$, в которой реализуются все типы над всеми подмножествами $X$ мощности $\lambda$ носителя $B_{\alpha}$ и которая является элементарным расширением $B_{\alpha}$.

Так как $\operatorname{cf}\left(2^{\lambda}\right)>\lambda$ (следствие 2.4$)$, то объединение возрастающей последовательности элементарных подсистем $B_{\alpha}\left(\alpha<2^{\lambda}\right)$ и является $\lambda^{+}$-насыщенным элементарным расширением системы $A$, которое имеет мощность $2^{\lambda}$.

ОПРЕДЕЛЕНИЕ 11 (специальной структуры). $L$-структура $M$ мощности $\lambda$ называется специальной, если $M$ является объединением возрастающей последовательности таких элементарных подсистем

$$
\left\{M_{\mu} \mid \mu \text { является кардиналом и } \mu<\lambda\right\} \text {, }
$$

что для каждого кардинала $\mu$, меньшего $\lambda$, подсистема $M_{\mu}$ является $\mu^{+}$-насыщенной. Эта возрастающая последовательность элементарных подсистем называется специализирующей для $M$.

Напомним, что $\mu^{+}$обозначает наименьший кардинал среди кардиналов, больших $\mu$.

Очевидно, что каждая насыщенная система является специальной.

Следующие две теоремы будут неоднократно использоваться дальше.

Теорема 3.10. Элементарно эквивалентные специалъные L-структуры A и В одной мощности $\lambda$ изоморфны.

ДокАЗАТЕЛЬство. В доказательстве теоремы 3.8 надо сделать некоторые усовершенствования. Пусть $|A|=\left\{a_{\alpha} \mid \alpha<\lambda\right\}$ и $|B|=\left\{b_{\alpha} \mid \alpha<\lambda\right\}$. Пусть, кроме того,

$$
\left\{A_{\mu} \mid \mu \text { является кардиналом и } \mu<\lambda\right\}
$$

и

$$
\left\{B_{\mu} \mid \mu \text { является кардиналом и } \mu<\lambda\right\}
$$

являются специализирующими последовательностями для $A$ и $B$. При построении $\tau_{\alpha}$ мы будем дополнительно требовать, чтобы элемент $\tau_{\alpha}\left(a_{\beta}\right)$ лежал в $B_{\mu}$, если $\beta<\mu$, и чтобы $\tau_{\alpha}^{-1}\left(b_{\beta}\right)$ лежал в $A_{\mu}$, если $\beta<\mu$. В остальном доказательство повторяет доказательство теоремы 3.8 .

Tеорема 3.11. Для любой бесконечной L-структуры $M$ и любого кардинала $\lambda$, большего как мощности сигнатуры $L$, так и мощности $M$ и удовлетворяющего условию $\lambda^{*}=\lambda$, существует специальная L-структура $N$ мощности $\lambda$, являющаяся элементарным расширением $L$-структуры $M$.

ДокАЗАТЕЛЬство. Если кардинал $\lambda$ не является предельным, $\lambda=\mu^{+}$, то из условия $\lambda^{*}=\lambda$ следует, что $2^{\mu}=\mu^{+}$. Тогда, по теореме 3.9 , существует насыщенная система $N$ мощности $\lambda$, являющаяся элементарным расширением $L$-структуры $M$.

Рассмотрим случай, когда кардинал $\lambda$ является предельным. В этом случае $\mu^{+}<\lambda$ и $2^{\mu} \leqslant \lambda$ для любого кардинала $\mu$, меньшего $\lambda$. 
Для кардиналов $\mu$, меньших $\lambda$, но не меньших $\kappa$, где $\kappa$ является мощностью $L$-структуры $M$, будем строить $L$-структуры $N_{\mu}$ так, чтобы мощность $N_{\mu}$ равнялась $2^{\mu}$, чтобы $N_{\mu}$ были $\mu^{+}$-насыщенными и чтобы они образовывали возрастающую последовательность элементарных подсистем, каждая из которых является элементарным расширением $M$. Для этого построения используется теорема 3.9. Для $\mu$, меньших $\kappa$, в качестве $N_{\mu}$ возьмем $N_{\kappa}$. Объединение построенной последовательности

$$
\left\{N_{\mu} \mid \mu \text { является кардиналом и } \mu<\lambda\right\}
$$

и является искомой специальной системой. Теорема 3.11 доказана.

Легко заметить, что любое обеднение $\lambda$-насыщенной системы является $\lambda$ насыщенной системой. По этой причине любое обеднение специальной системы является специальной системой.

Как уже упоминалось во введении, отношение $<$ на множестве $I$ называется отношением линейного порядка, если для любых элементов $a, b$ и $c$ из $I$ выполняются условия:

(a) либо $a<b$, либо $b<a$, либо $a=b$ (тотальность);

(b) если $a<b$ и $b<c$, то $a<c$ (транзитивность);

(c) если $a<b$, то ни одно из условий $a=b$ и $b<a$ не выполняется (антисимметричность).

Линейный порядок $(I,<)$ называется плотным, если для каждых $a<b$ из $I$ найдется такой элемент $c$ из $I$, что $a<c<b$.

Линейный порядок $(I,<)$ называется полным, если при любом таком разбиении $I$ на два непустых подмножества, что каждый элемент первого подмножества меньше каждого элемента второго подмножества, а каждый элемент $I$ входит в одно из этих подмножеств, либо в первом подмножестве есть наибольший элемент, либо во втором подмножестве есть наименьший элемент.

ОПРЕДЕЛЕНИЕ 12 (неразличимой последовательности). Подмножество $I$ носителя $L$-структуры $M$, линейно упорядоченное отношением $<$, называется $\theta$ неразличимой последовательностью в $M$ по отношению к < для $L$-формулы $\theta\left(x_{1}, \ldots, x_{n}\right)$, если для каждых двух кортежей $\bar{a}$ и $\bar{b}$ длины $n$, составленных из элементов множества $I$ и удовлетворяющих условиям $a_{1}<\cdots<a_{n}$ и $b_{1}<\cdots<b_{n}, \theta(\bar{a})$ истинно в $M$ тогда и только тогда, когда $\theta(\bar{b})$ истинно в $M$.

Линейно упорядоченное отношением < подмножество $I$ носителя $L$-структуры $M$ называется неразличимой последовательностъю в $M$ по отношению к $<$, если для каждого натурального $n$ и каждой $L$-формулы $\theta\left(x_{1}, \ldots, x_{n}\right)$ множество $I$ является $\theta$-неразличимой последовательностью.

Неразличимая последовательность $I$ называется эффективной, если существует алгоритм, который по $L$-формуле $\theta\left(x_{1}, \ldots, x_{n}\right)$ и по $a_{1}<\cdots<a_{n}$ из $I$ определяет, истинно ли $\theta(\bar{a})$ в $M$.

Непосредственным следствием теоремы Рамсея (теорема 2.6) является

ТеОрема 3.12. Пусть $M$ - бесконечная L-структура, $a<-$ линейное упорядочение ее универсума. Для каждой $L$-формулы $\theta\left(x_{1}, \ldots, x_{n}\right)$ существует 
бесконечное подмножество I универсума структуры $M$, которое являтся $\theta$-неразличимой последовательностью в $M$ по отношению $\kappa<$.

Из этой теоремы и теоремы компактности Мальцева (теорема 3.5) легко получается следующий результат.

ТЕОРЕмА 3.13 (о существовании неразличимой последовательности). Для любой линейно упорядоченной отношением $<$, имя которого входит в L, бесконечной $L$-структуры $M$, носитель которой не имеет общих элементов $c$ множеством I, и любого линейного порядка $(I,<)$ существует такое элементарное расширение $N$ структуры $M$, что носитель L-структуры $N$ содержит I и I является неразличимой последовательностью в $N$ по отношению $\kappa<, a(I,<)$ является подсистемой $\{<\}$-обеднения L-структуры $N$.

\section{4. Определения}

Под универсумом сигнатуры $L$ понимается любая бесконечная алгебраическая система сигнатуры $L$. Ради краткости обозначений мы для заданного универсума $U$ через $U$ будем часто обозначать и носитель универсума $U$. Обычно мы предполагаем, что сигнатура рассматриваемого универсума конечна. Как уже упоминалось во введении, мы будем рассматривать только линейно упорядоченные универсумы. Так называют универсумы, для которых среди основных отношений универсума есть бинарное отношение, которое является отношением линейного порядка (тотальным, транзитивным и антисимметричным отношением). Мы будем использовать < как имя для этого линейного порядка.

Схемой базы данных является конечный набор имен отношений и выделенных элементов с указанием числа аргументных мест каждого отношения. Все отношения, имена которых включены в рассматриваемую схему базы данных, предполагаются конечноместными. Таким образом, схема базы данных представляет собой некоторую другую сигнатуру, в которой нет символов операций, отличных от символов выделенных элементов. Мы всегда предполагаем, что схема базы данных не имеет общих символов с сигнатурой универсума.

Через $(L, \tau)$ будем обозначать сигнатуру, полученную добавлением к символам сигнатуры $L$ символов сигнатуры $\tau$, не меняя при этом число аргументных мест ни у одного символа.

Для заданного универсума $U$ состоянием рассматриваемой схемы $\rho$ базы данных, или $\rho$-состоянием, или базой данных с рассматриваемой схемой $\rho$ называется отображение, которое каждому имени отношения местности $k$ из рассматриваемой схемы $\rho$ ставит в соответствие конкретное отношение той же местности на $U$, которое можно рассматривать как подмножество множества $U^{k}$, которое представляет собой набор последовательностей длины $k$ элементов универсума и может быть задано как таблица с $k$ столбцами и некоторым множеством строк, а каждому имени выделенного элемента это отображение в качестве значения приписывает конкретный элемент универсума. Состояние $s$ обогащает универсум $U$ сигнатуры $L$ до $(L, \rho)$-структуры, которую мы будем обозначать как $(U, s)$.

Активная область состояния $s$ - это множество, состоящее из всех элементов всех строк всех таблиц из $s$ и значений всех символов выделенных 
элементов для этого состояния. Активная область состояния $s$ обозначается через $\mathrm{AD}(s)$. Состояние $s$ называется конечным, если его активная область является конечным множеством, другими словами, отношения из $s$ представляют собой конечные наборы последовательностей соответствующей длины элементов универсума и могут быть заданы конечными таблицами.

Состояние $s$ называется состоянием над $I$, если $\mathrm{AD}(s) \subseteq I$, т.е. каждому имени отношения из рассматриваемой схемы базы данных поставлено в соответствие отношение той же местности на множестве $I$, а значением каждого символа выделенного элемента из рассматриваемой схемы базы данных является элемент множества $I$.

В качестве языка запросов мы используем язык логики предикатов первого порядка. Это значит, что запросами являются формулы этого языка. В формулах можно использовать либо только имена рассматриваемой схемы $\rho$ и символ < отношения порядка, либо также и все другие символы операций и отношений из сигнатуры $L$ универсума. Формулы первого вида мы называем ограниченными ${ }^{2} \rho$-запросами, а формулы второго вида - расширенными ${ }^{3} \rho$-запросами.

ОПРЕДЕЛЕНИЕ 13 (ограниченных и расширенных запросов). Ограниченные $\rho$-запросы - это $(<, \rho)$-формулы, а расширеннъе $\rho$-запросы - это $(L, \rho)$-формулы.

Если формула, задающая запрос, является замкнутой (не содержит свободных переменных), то запрос называется булевым.

ТеОрема 4.1. Пусть $\rho$ состоит из символа $P$, являющегося символом одноместного отношения. Не существует булева ограниченного $\rho$-запроса, истинного для заданного $\rho$-состояния тогда и только тогда, когда активная область этого состояния содержит четное число элементов.

ДокАЗАТЕЛЬСтво. От противного. Пусть такой запрос существует и задается замкнутой $(<, \rho)$-формулой $\phi$. Можно считать, что формула $\phi$ является предваренной и содержит $k$ кванторов. Будем предполагать, что $\phi$ есть

$$
\left(Q_{1} x_{1}\right) \ldots\left(Q_{k} x_{k}\right) \psi\left(x_{1}, \ldots, x_{k}\right),
$$

где $\left(Q_{1} x_{1}\right) \ldots\left(Q_{k} x_{k}\right)$ - кванторная приставка.

Введем понятие расстояния между элементами универсума в заданном подмножестве $P$ универсума. Расстояние от $a$ до $a$ в любом подмножестве универсума равно 0 . Пусть $a$ и $b$ различны и $a<b$. Расстоянием от $a$ до $b$ в подмножестве $P$ универсума назовем увеличенное на единицу число элементов универсума, лежащих в рассматриваемом подмножестве $P$ между рассматриваемыми элементами. Будем обозначать это расстояние с помощью $\rho^{P}(a, b)$. Если подмножество не указано, то оно считается всем универсумом. В случае бесконечного подмножества расстояние может быть бесконечным. Если $b<a$, то $\rho(a, b)=-\rho(b, a)$.

Выберем в универсуме подмножество $A$, содержащее $2^{2 k+2}$ элементов, расстояние между любыми различными двумя из которых больше $2^{2 k+2}$ и каждый

\footnotetext{
${ }^{2}$ Англ. restricted.

${ }^{3}$ Англ. extended.
} 
из которых отстоит от любого из концов универсума, если универсум имеет хотя бы один конец, больше, чем на $2^{2 k+2}$. Например, если универсум плотно упорядочен и не имеет концов, то выберем в универсуме произвольное подмножество $A$, содержащее $2^{2 k+2}$ элементов. Пусть состояние $s_{1}$ ставит $P$ в соответствие все это подмножество $A$, а состояние $s_{2}$ ставит $P$ в соответствие множество $B$, полученное из $A$ выбрасыванием одного немаксимального и неминимального элемента.

Легко проверяется, что эти состояния не различаются формулой $\phi$. Но в первом состоянии число элементов $P$ четно, а во втором - нечетно. По этой причине формула $\phi$ не разделяет состояния с четным и нечетным числом элементов активной области.

Для проверки обратной индукцией по $i$ доказываем более общее утверждение. Для его формулировки введем следующие определения.

$i$-обогащением состояния $s_{1}$ назовем состояние, которое для $j=1, \ldots, i$ символу $c_{j}$ ставит в соответствие элемент $a_{j}$. $i$-обогащением состояния $s_{2}$ назовем состояние, которое для $j=1, \ldots, i$ символу $c_{j}$ ставит в соответствие элемент $b_{j}$.

Назовем выделенными элементы $a_{1}, \ldots, a_{i}$, концы универсума, концы $A$. Кроме того, если $a$ - произвольный выделенный элемент не из $A$, то ближайшие к нему элементы $A$ тоже считаем выделенными. Образом конца $A$ или конца универсума является сам этот конец. Образом элемента $a_{j}$ из $a_{1}, \ldots, a_{i}$ назовем $b_{j}$. Если $a_{*}$ и $a^{*}-$ ближайшие слева и справа к выделенному $a \notin A$ элементы множества $A$, то их образами будут ближайшие к образу а слева и справа элементы $b_{*}$ и $b^{*}$ множества $B$. Образом интервала, заданного двумя выделенными элементами, является интервал, заданный образами этих выделенных элементов. Образ выделенного элемента $a$ обозначается через $s(a)$. Пусть $\varepsilon_{i}=2^{k+1-i}-1$.

Лемма 4.2. Пусть $i$-обогащения состояний $s_{1} u s_{2}$ удовлетворяют следующим условиям для любых выделенных в $s_{1}$ элементов $a^{\prime}, a^{\prime \prime}$ и выделенных в $s_{2}$ образов этих элементов $b^{\prime}, b^{\prime \prime}$ :

1) $\left|\rho\left(a^{\prime}, a^{\prime \prime}\right)\right| \geqslant \varepsilon_{i}$ тогда и только тогда, когда $\left|\rho\left(b^{\prime}, b^{\prime \prime}\right)\right| \geqslant \varepsilon_{i}$;

2) $\left|\rho^{A}\left(a^{\prime}, a^{\prime \prime}\right)\right| \geqslant \varepsilon_{i}$ тогда и только тогда, когда $\left|\rho^{B}\left(b^{\prime}, b^{\prime \prime}\right)\right| \geqslant \varepsilon_{i}$;

3) если $\left|\rho\left(a^{\prime}, a^{\prime \prime}\right)\right|<\varepsilon_{i}$, mo $\rho\left(b^{\prime}, b^{\prime \prime}\right)=\rho\left(a^{\prime}, a^{\prime \prime}\right)$;

4) если $\left|\rho^{A}\left(a^{\prime}, a^{\prime \prime}\right)\right|<\varepsilon_{i}$, mo $\rho^{B}\left(b^{\prime}, b^{\prime \prime}\right)=\rho^{A}\left(a^{\prime}, a^{\prime \prime}\right)$;

5) $a^{\prime}<a^{\prime \prime}$ тогда и только тогда, когда $b^{\prime}<b^{\prime \prime}$;

6) $a^{\prime} \in A$ тогда и только тогда, когда $b^{\prime} \in B$.

Тогда і-обогащения состояний $s_{1}$ u $s_{2}$ не различаются формулой

$$
\left(Q_{i+1} x_{i+1}\right) \ldots\left(Q_{k} x_{k}\right) \psi\left(c_{1}, \ldots, c_{i}, x_{i+1}, \ldots, x_{k}\right) .
$$

ДокАЗАТЕЛЬСтво ЛЕммы. Лемма доказывается обратной индукцией по $i$. При $i=k$ из условий 5) и 6 ) следует, что $k$-обогащения состояний $s_{1}$ и $s_{2}$ не различаются бескванторной формулой

$$
\psi\left(c_{1}, \ldots, c_{i}, c_{i+1}, \ldots, c_{k}\right) .
$$

Поэтому лемма верна для $i=k$.

Пусть $i<k$ и для $i+1$ лемма верна. Пусть для $i$ выполнены все условия 1)-6). 
Нам надо доказать, что либо формула (2) истинна одновременно и для $i$ обогащения $s_{1}$, и для $i$-обогащения $s_{2}$, либо формула (2) ложна одновременно и для $i$-обогащения $s_{1}$, и для $i$-обогащения $s_{2}$. Предположим противное. Тогда для одного из рассматриваемых состояний рассматриваемая формула истинна. Мы докажем, что эта формула истинна и для другого из этих состояний, что и закончит доказательство леммы.

Пусть формула (2) истинна для $i$-обогащения $s_{1}$. Если эта формула истинна для другого из рассматриваемых состояний, рассуждение совершенно аналогично.

Случай 1. $Q_{i+1}$ есть $\exists$. Выберем такой элемент $a_{i+1}$, для которого при $s_{1}\left(c_{i+1}\right)=a_{i+1}$ для этого $(i+1)$-обогащения состояния $s_{1}$ истинна формула

$$
\left(Q_{i+2} x_{i+2}\right) \ldots\left(Q_{k} x_{k}\right) \psi\left(c_{1}, \ldots, c_{i}, c_{i+1}, x_{i+2}, \ldots, x_{k}\right) .
$$

Теперь выберем элемент $b_{i+1}$ так, чтобы условия 1)-6) при $s_{2}\left(c_{i+1}\right)=b_{i+1}$ выполнялись с заменой $i$ на $i+1$. Если мы сможем это сделать, то по индукционному предположению $(i+1)$-обогащения состояний $s_{1}$ и $s_{2}$ не будут различаться формулой (3) и формула (2) будет истинна и для $i$-обогащения $s_{2}$.

Если $a_{i+1}$ совпадает с одним из выделенных элементов, то пусть $b_{i+1}$ совпадает с образом этого элемента. В этом случае выполнимость условий 1)-6) при $s_{2}\left(c_{i+1}\right)=b_{i+1}$ с заменой $i$ на $i+1$ очевидна.

Далее предполагаем, что $a_{i+1}$ отличен от всех выделенных элементов.

Выделенные элементы разбивают универсум на конечное число интервалов, и $a_{i+1}$ попадает в один из них. Мы будем выбирать $b_{i+1}$ в соответствующем интервале. При соблюдении этих правил условие 5) заведомо выполнено.

Пусть $a_{*}$ и $a^{*}$ - это такие соседние из выделенных элементов, между которыми лежит $a_{i+1}$. Случай, когда $a_{i+1}$ выбран меньше самого меньшего из выделенных элементов или больше самого большего из них, мы отдельно не рассматриваем. Этот случай рассматривается аналогично.

Случай 1.1. $a_{i+1} \in A$.

Случай 1.1.1. Если $\rho^{A}\left(a_{*}, a_{i+1}\right)$ или $\rho^{A}\left(a_{i+1}, a^{*}\right)$ меньше $\varepsilon_{i+1}$, то в качестве $b_{i+1}$ берем элемент $B$, находящийся на таком же расстоянии в $B$ от $s\left(a_{*}\right)$ или $s\left(a^{*}\right)$ соответственно.

Если при этом элемент $a_{i+1}$ был близок в $A$ к еще какому-то выделенному элементу $s_{1}$, например, $a$, то если $\rho^{A}\left(a_{*}, a_{i+1}\right)<\varepsilon_{i+1}$, получаем:

$$
\begin{aligned}
\left|\rho^{A}\left(a, a_{*}\right)\right| & =\left|\rho^{A}\left(a, a_{i+1}\right)+\rho^{A}\left(a_{i+1}, a_{*}\right)\right| \\
& \leqslant\left|\rho^{A}\left(a, a_{i+1}\right)\right|+\left|\rho^{A}\left(a_{i+1}, a_{*}\right)\right|<2 \varepsilon_{i+1}<\varepsilon_{i} .
\end{aligned}
$$

Тогда, согласно 2) и 4), $\rho^{B}\left(s(a), s\left(b_{*}\right)\right)=\rho^{A}\left(a, a_{*}\right)$ и

$$
\begin{aligned}
\rho^{B}\left(s(a), b_{i+1}\right) & =\rho^{B}\left(s(a), s\left(a_{*}\right)\right)+\rho^{B}\left(s\left(a_{*}\right), b_{i+1}\right) \\
& =\rho^{A}\left(a, a_{*}\right)+\rho^{A}\left(a_{*}, a_{i+1}\right)=\rho^{A}\left(a, a_{i+1}\right) .
\end{aligned}
$$

Теперь рассмотрим расстояния между $a_{i+1}$ и элементом $a_{*}$, а также их образами в универсуме. Очевидно, достаточно рассмотреть только случай, когда $a_{*} \notin A$, так как в противном случае $\rho\left(a_{*}, a_{i+1}\right) \geqslant 2^{2 k+2}$ и то же самое неравенство выполнено для образов. Если $\rho^{A}\left(a_{*}, a_{i+1}\right) \geqslant 2$, то $\rho\left(a_{*}, a_{i+1}\right) \geqslant 2^{2 k+2} \geqslant \varepsilon_{i}$ 
и то же самое выполнено для образов. Пусть $\rho^{A}\left(a_{*}, a_{i+1}\right)=1$. Но тогда $a_{i+1}$ был бы выделенным элементом как ближайший к $a_{*}$ элемент множества $A$.

Случай 1.1.2. Если же оба расстояния $\rho^{A}\left(a_{*}, a_{i+1}\right)$ и $\rho^{A}\left(a_{i+1}, a^{*}\right)$ больше или равны $\varepsilon_{i+1}$, то, очевидно, $\rho^{A}\left(a_{*}, a^{*}\right) \geqslant 2 \varepsilon_{i+1}$. Тогда, согласно 2$)$ и 4$)$,

$$
\rho^{B}\left(s\left(a_{*}\right), s\left(a^{*}\right)\right) \geqslant 2 \varepsilon_{i+1},
$$

и существует элемент $B$, находящийся и от $s\left(a_{*}\right)$, и от $s\left(a^{*}\right)$ на расстоянии, не меньшем $\varepsilon_{i+1}$; этот элемент и нужно взять в качестве $b_{i+1}$.

Случай 1.2. $a_{i+1} \notin A$.

Случай 1.2.1. Если $\rho\left(a_{*}, a_{i+1}\right)$ или $\rho\left(a_{i+1}, a^{*}\right)$ меньше $\varepsilon_{i+1}$, то в качестве $b_{i+1}$ берем элемент универсума, находящийся на таком же расстоянии от $s\left(a_{*}\right)$ или $s\left(a^{*}\right)$ соответственно. Прочие рассуждения такие же, как в случае $1.1 .1 \mathrm{c}$ заменой $\rho^{A}$ и $\rho^{B}$ на $\rho$.

Случай 1.2.2. В противном случае пусть $a_{* *}$ - ближайший слева к $a_{i+1}$ элемент $A$, а $a^{* *}$ - ближайший справа. Если хотя бы одно из расстояний $\rho^{A}\left(a_{*}, a_{* *}\right)$ или $\rho^{A}\left(a^{* *}, a^{*}\right)$ меньше $\varepsilon_{i+1}$, то строим $s\left(a_{* *}\right)$ и $s\left(a^{* *}\right)$ так же, как в случае 1.1.1. Если оба эти расстояния больше или равны $\varepsilon_{i+1}$, то $\rho^{A}\left(a_{*}, a^{*}\right) \geqslant 2 \varepsilon_{i+1}+1=\varepsilon_{i}$. Тогда между $s\left(a_{*}\right)$ и $s\left(a^{*}\right)$ тоже можно найти два подряд идущих элемента $B$, удаленных от $s\left(a_{*}\right)$ и $s\left(a^{*}\right)$ в $B$ не менее чем на $\varepsilon_{i+1}$. Возьмем их в качестве $s\left(a_{* *}\right)$ и $s\left(a^{* *}\right)$.

Теперь можем считать, что между $a_{i+1}$ и ближайшими к нему выделенными элементами нет элементов $A$. Если после этого примени́м случай 1.2 .1 , то действуем, как указано там. Иначе оба расстояния до ближайших выделенных элементов больше $\varepsilon_{i+1}$. Тогда и соответствующее расстояние между образами ближайших выделенных элементов в $s_{2}$ больше $\varepsilon_{i}$ и можно найти элемент в $s_{2}$, находящийся достаточно далеко от образов этих ближайших выделенных элементов и между этими образами.

Случай 2. $Q_{i+1}$ есть $\forall$, а для $i$-обогащения состояния $s_{2}$ формула (2) ложна. Выберем такой элемент $b_{i+1}$, для которого при $s_{2}\left(c_{i+1}\right)=b_{i+1}$ для $(i+1)$-обогащения состояния $s_{2}$ формула (3) ложна. Рассуждая так же, как мы рассуждали в случае 1 , выберем такой элемент $a_{i+1}$, для которого при $s_{1}\left(c_{i+1}\right)=a_{i+1}$ для $(i+1)$-обогащения состояния $s_{1}$ ложна формула (3). Это, однако, противоречит предположению об истинности формулы (2) для $i$-обогащения $s_{1}$. Лемма 4.2 доказана.

Из доказанной леммы при $i=0$ следует, что состояния $s_{1}$ и $s_{2}$ не различаются формулой $\phi$. Теорема 4.1 доказана.

Иногда мы будем рассматривать частичные изоморфизмы обеднений изучаемых систем. В таком случае важно отмечать, в какой обедненной сигнатуре рассматриваемое отображение является частичным изоморфизмом. Для этого мы используем понятие частичного $L_{1}$-изоморфизма. Это означает, что рассматриваются обеднения изучаемых структур до систем сигнатуры $L_{1}$ и частичный изоморфизм между ними. В частности, частичный <-изоморфизм является частичным изоморфизмом <-обеднений рассматриваемых структур.

Более подробно, одно-однозначное отображение $f$ называется частичным <изоморфизмом подмножества $X$ носителя системы $U$ в $U$ и обозначается как 
$f: X \rightarrow U$, если $f$ одно-однозначно отображает $X$ в носитель системы $U$ и для каждых $x$ и $y$ из $X$ тогда и только тогда $x<y$ в $U$, когда $f(x)<f(y)$ в $U$. Если $X$ совпадает с носителем системы $U$, то $f$ называется <-автоморфизмом системы $U$.

Мы рассматриваем только формулы специального вида, которые задают так называемые локально генерические запросы.

ОПРЕДЕЛЕНИЕ 14 (локально генерического запроса). Запрос $\Phi(\bar{x})$ называется локалъно генерическим для состояний из класса $K$ (или относительно состояний из класса $K)$, если

$$
(U, s) \models \Phi(\bar{a}) \Leftrightarrow(U, f(s)) \models \Phi(f(\bar{a}))
$$

для любого такого частичного <-изоморфизма $f: X \rightarrow U$, что $X \subseteq U$, для любого такого состояния $s$ над $X$ из класса $K$, что $f(s)$ лежит в $K$, и для любой последовательности $\bar{a}$ элементов $X$.

Здесь образ последовательности есть последовательность образов, а образ таблицы является таблицей, составленной из образов элементов заданной таблицы. Образ состояния (конечной совокупности таблиц) представляет собой совокупность образов этих таблиц. Напомним, что если состояние $s$ является состоянием над $X$, то $\mathrm{AD}(s) \subseteq X$. По этой причине $f(s)$ определено.

Если $K$ является классом всех конечных состояний, то говорят о локальной генеричности запроса для конечных состояний (или просто о локальной генеричности).

ОПРЕДЕЛЕНИЕ 15 (генерического запроса). Запрос $\Phi(\bar{x})$ называется генерическим для состояний из класса $K$ (или относительно состояний из класса $K$ ), если

$$
(U, s) \models \Phi(\bar{a}) \Leftrightarrow(U, f(s)) \models \Phi(f(\bar{a}))
$$

для любого <-автоморфизма $f$ универсума $U$ и для любого такого состояния $s$ из класса $K$, что $f(s)$ лежит в $K$.

Замкнутая $L$-формула короче называется $L$-высказыванием.

Для $(L, \rho)$-высказывания $\psi$ и натурального числа $m$ легко строится такое $L$ высказывание $\psi_{m}$, что для любой $L$-структуры $V$ это высказывание $\psi_{m}$ справедливо в $V$ тогда и только тогда, когда $\psi$ выполняется для всех $\rho$-состояний над $V$, активная область которых содержит не более $m$ элементов.

Мы включаем $(L, \rho)$-высказывание $\psi$ в множество $\operatorname{Fin}(V, \rho)$ тогда и только тогда, когда $\psi_{m}$ принадлежит $\operatorname{Th}(V)$ для любого натурального $m$. Понятно, что $W \equiv V$ влечет

$$
\operatorname{Fin}(V, \rho)=\operatorname{Fin}(W, \rho) .
$$

По этой причине $(L, \rho)$-теория $\operatorname{Fin}(T, \rho)$ корректно определяется как $\operatorname{Fin}(W, \rho)$, где $W$ есть произвольная модель полной $L$-теории $T$.

ОПРЕДЕЛЕНИЕ 16 (псевдоконечного состояния). $\rho$-состояние $s$ для $L$-структуры $W$ называется псевдоконечным в $W$, если $(W, s)$ есть модель $(L, \rho)$-теории $\operatorname{Fin}(W, \rho)$. 


\section{5. Критерий транслируемости расширенного запроса в ограниченный}

Следующая теорема заимствована из [3]. Запись $\phi \leftrightarrow \psi$ здесь и далее является сокращением для $(\phi \rightarrow \psi) \wedge(\psi \rightarrow \phi)$.

ТеОРема 5.1. Для любого универсума $U$ и любого расширенного булевого $\rho$-запроса ф следующие три условия эквивалентны:

1) существует ограниченный $\rho$-запрос $\psi$, эквивалентный в $U$ запросу $\phi$ для конечных состояний базы данных;

2) запрос ф является генерическим для всех псевдоконечных состояний в $V$ для всех $V \equiv U$;

3) для некоторой несчетной мощности $\kappa$, удовлетворяющей условию $\kappa=$ $\kappa^{*}$, запрос ф является генерическим для всех псевдоконечных состояний в $V$ для специальной $V \equiv U$ мощности к.

ДоказАтельство. 1) $\Rightarrow 2$ ). Допустим, что $\phi$ эквивалентен в $U$ для конечных состояний ограниченному запросу $\psi$. Тогда $\phi \leftrightarrow \psi$ лежит в $\operatorname{Fin}(U, \rho)$ и, значит, в $\operatorname{Fin}(V, \rho)$ для каждого $V \equiv U$. Так как каждый ограниченный запрос является генерическим относительно любых состояний, то $\psi$ является генерическим относительно любых состояний $V$. Поэтому $\phi$ является генерическим относительно псевдоконечных состояний $V$.

$2) \Rightarrow 3)$ выполняется очевидным образом.

$3) \Rightarrow 1)$. Пусть $T=\operatorname{Th}(U)$.

Пусть

$$
\rho=\left\{R_{1}, \ldots, R_{n}, c_{1}, \ldots, c_{k}\right\}
$$

Выберем

$$
\rho^{\prime}=\left\{R_{1}^{\prime}, \ldots, R_{n}^{\prime}, c_{1}^{\prime}, \ldots, c_{k}^{\prime}\right\}
$$

как копию схемы $\rho$, предполагая, что схемы $\rho$ и $\rho^{\prime}$ не имеют общих элементов, а для любого $i \in\{1, \ldots, n\}$ число аргументных мест у $R_{i}$ и $R_{i}^{\prime}$ одинаково. Для $(L, \rho)$-высказывания $\theta$ через $\theta\left(\rho^{\prime}\right)$ обозначим его $\left(L, \rho^{\prime}\right)$-копию. Подробнее, $\theta\left(\rho^{\prime}\right)$ получается из $\theta$ заменой каждого вхождения $R_{i}$ и $c_{j}$ на вхождение $R_{i}^{\prime}$ или $c_{j}^{\prime}$ соответственно. В частности, $\theta(\rho)$ совпадает с $\theta$. Пусть $\bar{\rho}=\rho \cup \rho^{\prime}$.

Сначала мы замечаем, что из 3) следует несовместность следующей теории Г:

$$
\operatorname{Fin}(T, \bar{\rho}) \cup\left\{\theta(\rho) \leftrightarrow \theta\left(\rho^{\prime}\right): \theta \text { есть }(<, \rho) \text {-высказывание }\right\} \cup\left\{\phi(\rho), \neg \phi\left(\rho^{\prime}\right)\right\} \text {. }
$$

Пусть, напротив, $\left(W, r, r^{\prime}\right)$ - модель Г. Используя теорему ЛёвенгеймаСкулема и теорему 3.11, можно предполагать, что эта модель специальна и имеет мощность $\kappa$.

Тогда ее обеднения $W,(W \uparrow\{<\}, r)$ и $\left(W \uparrow\{<\}, r^{\prime}\right)$ тоже являются специальными системами. Поэтому $W$ и $V$ являются элементарно эквивалентными специальными системами одной и той же мощности. По теореме $3.10 \mathrm{~W}$ и $\mathrm{V}$ изоморфны.

Из Г также следует, что системы $(W \uparrow\{<\}, r)$ и $\left(W \uparrow\{<\}, r^{\prime}\right)$ элементарно эквивалентны. Они тоже имеют одну и ту же мощность. Поэтому они тоже 
изоморфны. Значит, существует <-автоморфизм системы $W$, который переводит $r$ в $r^{\prime}$. Так как $\left(r, r^{\prime}\right)$ является псевдоконечным состоянием в $W, \phi(\rho)$ истинно в $(W, r)$, но $\phi\left(\rho^{\prime}\right)$ ложно в $\left(W, r^{\prime}\right)$, то запрос $\phi$ не является генерическим для псевдоконечных состояний в $W$. А так как $V$ и $W$ изоморфны, это противоречит условию 3).

По теореме компактности Мальцева (теорема 3.5) Г имеет конечную несовместную часть. Это означает, что для некоторых $m<\omega$ и $(<, \rho)$-высказываний $\theta_{0}, \ldots, \theta_{m-1}$ выполняется

$$
\operatorname{Fin}(T, \bar{\rho}), \quad \bigwedge_{i<m}\left(\theta_{i}(\rho) \leftrightarrow \theta_{i}\left(\rho^{\prime}\right)\right), \quad \phi(\rho) \vdash \phi\left(\rho^{\prime}\right)
$$

(если в некоторой $(L, \bar{\rho})$-структуре истинны все левые формулы, то в этой структуре истинна и правая формула). Пусть $\theta^{1}=\theta, \theta^{0}=\neg \theta$. Очевидно, что тогда для некоторых $\tau_{i j} \in\{0,1\}$ расширенный запрос $\phi$ эквивалентен относительно конечных состояний $U$ ограниченному запросу

$$
\bigvee_{i<l} \bigwedge_{j<m} \theta_{i}^{\tau_{i j}}
$$

\section{6. Относительные свойства псевдоконечной однородности и изолированности}

Материал этого раздела заимствован из [11].

ТеОРема 6.1. Пусть $U$ - произвольный универсум сигнатуры L, а расширенный булев $\rho$-запрос ф является локально генерическим для конечных состояний над $U$.

Следующие два условия эквивалентны:

1) для некоторой несчетной мощности к, удовлетворяющей условию $\kappa=\kappa^{*}$, существует такая специальная модель $(V, J)$ мощности $\kappa$, что $J$ является неразличимой последовательностью в $V, V \equiv U$ u запрос $\phi$ является локально генерическим относительно псевдоконечных состояний над $J$ в $V$;

2) ф эквивалентна в $U$ для конечных состояний над $U$ некоторой ограниченной $\rho$-формуле.

ДокАЗАтЕЛЬСтво. Пусть $\kappa$ и $(V, J)$ удовлетворяют условиям теоремы. Это означает, что $\kappa$ является несчетной мощностью, удовлетворяющей условию $\kappa=$ $\kappa^{*}$, специальная модель $(V, J)$ имеет мощность $\kappa, J$ является неразличимой последовательностью в $V$ и $V \equiv U$.

Лемма 6.2. Пусть частичный <-изоморфизм $g$ в $V$ переводит псевдоконечное состояние $p$ над $J$ в $V$ схемы $\rho$ в псевдоконечное состояние $p^{\prime}$ над $J$ в $V$ этой же схемы. Тогда g продолжается до <-автоморфизма $h$ системы $V \uparrow\{<\}$.

ДокАЗАТЕЛЬСтво. Заметим, что для каждого $a \in J$ множество

$$
\{x \in V \mid x<a \text { в } V\}
$$


имеет мощность $\kappa$. В самом деле, пусть $V_{\alpha}(\alpha<\kappa)$ является специализирующей последовательностью для $V$. Тогда найдется такой кардинал $\beta<\kappa$, что $a \in V_{\alpha}$ для всех $\beta<\alpha<\kappa$. Так как $V_{\alpha}$ является $\alpha^{+}$-насыщенной, то

$$
\left\{x \in V_{\alpha} \mid x<a \text { в } V_{\alpha}\right\}
$$

имеет мощность не меньше $\alpha$.

Аналогично доказывается, что для любых $a<b \in J$ множество

$$
\{x \in V \mid a<x<b \text { в } V\}
$$

имеет мощность $\kappa$ и что множество

$$
\{x \in V \mid a<x \text { в } V\}
$$

имеет мощность $\kappa$.

Так как обеднение любой специальной системы является специальной системой и так как $J$ является неразличимой последовательностью в $V$, то для $a, b \in J$ подструктуры

$$
(\{x \in V \mid x<a \text { в } V\},<)
$$

и

$$
(\{x \in V \mid x<b \text { в } V\},<)
$$

структуры $V \uparrow\{<\}$ являются элементарно эквивалентными специальными структурами одной мощности.

Поэтому эти структуры изоморфны. Пусть $h_{a, b}$ изоморфно отображает

$$
(\{x \in V \mid x<a \text { в } V\},<)
$$

на

$$
(\{x \in V \mid x<b \text { в } V\},<) .
$$

Аналогично, для $a, b \in J$ подструктуры

$$
(\{x \in V \mid a<x \text { в } V\},<)
$$

и

$$
(\{x \in V \mid b<x \text { в } V\},<)
$$

структуры $V \uparrow\{<\}$ являются элементарно эквивалентными специальными структурами одной мощности. Поэтому эти структуры изоморфны. Пусть $g_{a, b}$ изоморфно отображает

$$
(\{x \in V \mid a<x \text { в } V\},<)
$$

на

$$
(\{x \in V \mid b<x \text { в } V\},<) .
$$

Аналогично, для $a<b$ из $J$ и $c<d$ из $J$ подструктуры

$$
(\{x \in V \mid a<x<b \text { в } V\},<)
$$




$$
(\{x \in V \mid c<x<d \text { в } V\},<)
$$

структуры $V \uparrow\{<\}$ тоже изоморфны. Пусть $h_{a, b ; c, d}$ изоморфно отображает

$$
(\{x \in V \mid a<x<b \text { в } V\},<)
$$

на

$$
(\{x \in V \mid c<x<d \text { в } V\},<) .
$$

Теперь мы в состоянии построить <-автоморфизм $h$ системы $V \uparrow\{<\}$, продолжающий $g$. Пусть $u$ является наименьшим, а $v$ является наибольшим элементом в активной области состояния $p$. Для произвольного $x \in V$, не входящего в активную область состояния $p$, либо $x<u$, либо $v<x$, либо найдутся такие элементы ${ }_{x} a$ и $a_{x}$ в активной области состояния $p$, что ${ }_{x} a<x<a_{x}$ и между ${ }_{x} a$ и $a_{x}$ нет элементов активной области состояния $p$. В первом случае полагаем $h(x)=h_{u, g(u)}(x)$, во втором случае полагаем $h(x)=g_{v, g(v)}(x)$, в третьем случае полагаем $h(x)=h_{x} a, a_{x} ; g\left({ }_{x} a\right), g\left(a_{x}\right)(x)$. Лемма 6.2 доказана.

Пусть $\phi$ эквивалентна в $U$ для конечных состояний над $U$ некоторой ограниченной $\rho$-формуле.

Так как $\phi$ эквивалентна в $U$ для конечных состояний над $U$ некоторой замкнутой формуле сигнатуры $(<, \rho)$, то $\phi$ эквивалентна в $V$ для псевдоконечных состояний над $V$ некоторой замкнутой формуле сигнатуры $(<, \rho)$ и, значит, сохраняется при изоморфизмах структуры $(V \uparrow\{<\}, p)$, которые являются <-автоморфизмами, переводящими $p$ в псевдоконечное состояние структуры $V$. Так как $h$ из леммы 6.2 является таким <-автоморфизмом структуры $(V \uparrow\{<\}, p)$, переводящим $p$ в $p^{\prime}$, то истинность $\phi$ в $(V, p)$ равносильна истинности $\phi$ в $\left(V, p^{\prime}\right)$. Это означает, что запрос $\phi$ является локально генерическим относительно псевдоконечных состояний над $J$ в $V$.

Пусть теперь запрос $\phi$ является локально генерическим относительно псевдоконечных состояний над $J$ в $V$.

Предположим, что $\phi$ не эквивалентна в $U$ никакой ограниченной $\rho$-формуле для конечных состояний над $U$.

По теореме 5.1 тогда существуют такие модель $(W, I) \equiv(V, J)$ и псевдоконечное $\bar{\rho}$-состояние $\left(p, p^{\prime}\right)$ над $(W, I)$, что некоторый <-автоморфизм $h$ модели $W$ переводит $p$ в $p^{\prime}$, но при этом $(W, p) \models \phi(\rho)$ и $\left(W, p^{\prime}\right) \models \neg \phi\left(\rho^{\prime}\right)$. Можно предполагать, что $\left(W, I, p, p^{\prime}\right)$ является специальной моделью мощности $\kappa$. Но тогда $(W, I)=(V, J)$.

Пусть $\rho^{\prime}, \sigma$ и $\sigma^{\prime}$ - это такие копии $\rho$, что $\rho, \rho^{\prime}, \sigma, \sigma^{\prime}$ попарно не содержат общих символов. Пусть

$$
\eta=\rho \cup \rho^{\prime} \cup \sigma \cup \sigma^{\prime} \cup\left\{F, F^{\prime}\right\},
$$

где $F, F^{\prime}$ - новые бинарные символы отношений.

$(L, P)$-теорию структуры $(V, J)$ обозначим через $T$. Мы желаем доказать совместность совокупности $(L, P, \eta)$-высказываний $\Gamma$, которая утверждает существование такого $\eta$-состояния $\left(r, r^{\prime}, s, s^{\prime}, F_{0}, F_{0}^{\prime}\right)$ для некоторой модели $(L, P)$ теории $T$, для которого:

1) $\left(r, r^{\prime}\right)$ удовлетворяет $\operatorname{Th}\left(V, J, p, p^{\prime}\right)$; 
2) $\left(r, r^{\prime}, s, s^{\prime}, F_{0}, F_{0}^{\prime}\right)$ удовлетворяет $\operatorname{Fin}(T, \eta)$;

3) $F_{0}$ и $F_{0}^{\prime}$ являются частичными <-изоморфизмами, переводящими $r$ в $s$ и $r^{\prime}$ в $s^{\prime}$ соответственно;

4) $s$ и $s^{\prime}$ являются состояниями над $P$;

5) $s$ удовлетворяет $\phi(\sigma)$, и $s^{\prime}$ удовлетворяет $\neg \phi\left(\sigma^{\prime}\right)$.

Допустим, что Г совместна. Пусть $\left(W_{1}, J_{1}, r_{1}, r_{1}^{\prime}, s_{1}, s_{1}^{\prime}, F_{1}, F_{1}^{\prime}\right)$ является специальной моделью мощности $\kappa$ для $Г$. Тогда $\left(W_{1}, J_{1}\right)$ - специальная модель мощности $\kappa$. Так что можно предполагать, что $\left(W_{1}, J_{1}\right)=(V, J)$. Из 1$)$ следует, что $\left(W_{1}, r_{1}, r_{1}^{\prime}\right)$ и $\left(V, p, p^{\prime}\right)$ изоморфны. Пусть <-автоморфизм $h_{1}$ структуры $V$ переводит $r_{1}$ в $r_{1}^{\prime}$. Из 2) следует, что $\eta$-состояние $\left(r_{1}, r_{1}^{\prime}, s_{1}, s_{1}^{\prime}, F_{1}, F_{1}^{\prime}\right)$ псевдоконечно в $(V, J)$. Из 3$)$ следует, что частичный $<$-изоморфизм $g=F_{1}^{\prime} \circ h_{1} \circ F_{1}^{-1}$ переводит $s_{1}$ в $s_{1}^{\prime}$. Из 4) следует, что $s_{1}$ и $s_{1}^{\prime}$ являются состояниями над $J$. Из 5) следует, что $s_{1}$ удовлетворяет $\phi(\sigma)$, а $s_{1}^{\prime}$ не удовлетворяет $\phi\left(\sigma^{\prime}\right)$. Это означает, что $\phi$ не является локально генерическим для псевдоконечных состояний над $J$ в $V$, что противоречит выбору $(V, J)$.

По теореме компактности Мальцева, осталось доказать конечную совместность $\Gamma$. Достаточно для каждой $\gamma \in \mathrm{Th}\left(V, J, p, p^{\prime}\right)$ найти конечное $\eta$-состояние $\left(r, r^{\prime}, s, s^{\prime}, F, F^{\prime}\right)$ над $V$, которое удовлетворяет $\left.\left.\gamma, 3\right), 4\right)$ и 5$)$.

Так как $\left(p, p^{\prime}\right)$ является псевдоконечным над $(V, J)$, найдется конечное $\bar{\rho}$ состояние $\left(r, r^{\prime}\right)$ над $V$, удовлетворяющее $\gamma \wedge \phi(\rho) \wedge \neg \phi\left(\rho^{\prime}\right)$. Совсем просто найти $s, s^{\prime}, F, F^{\prime}$, удовлетворяющие 3) и 4). Так как $\phi$ локально генеричен для конечных состояний, выполняется 5). Это доказывает конечную совместность Г и завершает доказательство теоремы.

Пусть $A$ и $B$ - алгебраические системы сигнатуры $L$. Пусть $C$ - подмножество носителя системы $A$ и $D$ - подмножество носителя системы $B$. Пусть $h$ одно-однозначно отображает $C$ на $D$. Через $(B, h(a) \mid a \in C)$ обозначается такое обогащение $L$-структуры $B$ до $L(C)$-структуры, в котором значением добавленного имени элемента $а$ является $h(a)$. Отображение $h$ называется элементарным отображением из $A$ в $B$, если $(B, h(a) \mid a \in C)$ и $(A, a \mid a \in C)$ элементарно эквивалентны как алгебраические системы сигнатуры $L(C)$. Если $A$ совпадает с $B$, то $h$ называется элементарным отображением в $B$.

ОПРЕДЕЛЕНИЕ 17. Полная теория $T$ обладает первым свойством псевдоконечной однородности, если существуют модель $M$ полной теории $T$ и бесконечное множество $I$, которое является неразличимой последовательностью в $M$, такие, что

для всяких структуры $(N, J)$, элементарно эквивалентной структуре $(M, I)$, псевдоконечных подмножеств $A$ и $B$ множества $J$ в модели $N$, конечных подмножеств $C$ и $D$ модели $N$, элементарного в $N$ отображения $h$, одно-однозначно отображающего $(A \cup C)$ на $(B \cup D)$, с $\omega$-насыщенной $(N, A, B, h)$ и для любого $a \in N$ существует такой элемент $b \in N$, что $h \cup\{(a, b)\}$ является элементарным отображением в $N$. 
ОПРЕДЕЛЕНИЕ 18. Полная теория $T$ обладает вторым свойством псевдоконечной однородности, если существуют модель $M$ полной теории $T$ и бесконечное множество $I$, которое является неразличимой последовательностью в $(M, I)$, такие, что

для всякой структуры $(N, J)$, элементарно эквивалентной структуре $(M, I)$, псевдоконечных подмножеств $A$ и $B$ множества $J$ в модели $N$, конечных подмножеств $C$ и $D$ модели $N$, элементарного в $(N, J)$ отображения $h$, одно-однозначно отображающего $(A \cup C)$ на $(B \cup D)$, с $\omega$-насыщенной $(N, J, A, B, h)$ и для любого $a \in N$ существует такой элемент $b \in N$, что $h \cup\{(a, b)\}$ является элементарным отображением в $(N, J)$.

Теорема 6.3. Предположим, что теория универсума $U$ имеет первое (второе) свойство псевдоконечной однородности.

Тогда каждый расширенный локально генерический для конечных состояний над $U$ запрос ф эквивалентен для конечных состояний над U некоторому ограниченному запросу.

ДокАзАтельство. Оно следует доказательству теоремы 5.4 из [3]. Достаточно проверить, что $\phi$ удовлетворяет условию теоремы 6.1.

Пусть $\kappa=\kappa^{*}>\omega$. Пусть $(V, J) \equiv(M, I)$ и $(V, J)$ - специальная модель мощности $\kappa$.

Рассмотрим такие псевдоконечные $\rho$-состояния $r$ и $r^{\prime}$ над $J$ в $V$, что $r$ переводится в $r^{\prime}$ частичным <-изоморфизмом $g$ в $V$, область определения которого есть псевдоконечное в $V$ множество $A$, являющееся активной областью состояния $r$, а множество значений $g$ есть псевдоконечное множество $A^{\prime}$, являющееся активной областью состояния $r^{\prime}$.

Надо проверить, что $\phi$ выполняется в $(V, r)$ тогда и только тогда, когда $\phi$ выполняется в $\left(V, r^{\prime}\right)$.

При этом можно предполагать, что $\left(V, J, A, A^{\prime}, g\right)$ является $\omega$-насыщенной. Действительно, $\left(V, J, A, A^{\prime}, g\right)$ является $\omega$-насыщенной, если $\omega$-насыщенной является модель $\left(V, J, r, r^{\prime}, g\right)$. Рассмотрим специальную модель

$$
\left(V_{0}, I_{0}, r_{0}, r_{0}^{\prime}, g_{0}\right)
$$

мощности $\kappa$, элементарно эквивалентную $\left(V, J, r, r^{\prime}, g\right)$. Достаточно доказать утверждение для $\left(V_{0}, I_{0}, r_{0}, r_{0}^{\prime}, g_{0}\right)$. Последняя модель является $\omega$-насыщенной, так как $\operatorname{cf}(\kappa) \geqslant \omega$.

Достаточно показать, что $g$ является $(L, \rho)$-элементарным отображением из $(V, r)$ в $\left(V, r^{\prime}\right)$.

Но, благодаря $L$-неразличимости $J$ (соответственно $(L, P)$-неразличимости $J)$, отображение $g$ заведомо является $L$-элементарным $((L, P)$-элементарным) отображением и, кроме того, частичным $(L, \rho)$-изоморфизмом. По этой причине истинность каждой бескванторной формулы сигнатуры $(L, \rho)(A)$ (соответственно $(L, P, \rho)(A))$ в $(V, r, a \mid a \in A)$ (соответственно в $(V, J, r, a \mid$ $a \in A)$ ) совпадает с истинностью этой же формулы в $\left(V, r^{\prime}, g(a) \mid a \in A\right)$ (соответственно в $\left.\left(V, J, r^{\prime}, g(a) \mid a \in A\right)\right)$. 
Пусть истинность каждой предваренной формулы с меньшим числом кванторов сигнатуры $(L, \rho)(A)$ (соответственно $(L, P, \rho)(A))$ в

$$
(V, r, a \mid a \in A)
$$

(соответственно в $(V, J, r, a \mid a \in A))$ совпадает с истинностью этой же формулы B

$$
\left(V, r^{\prime}, g(a) \mid a \in A\right)
$$

(соответственно в $\left.\left(V, J, r^{\prime}, g(a) \mid a \in A\right)\right)$.

Надо доказать, что это утверждение верно и для предваренных формул с заданным числом кванторов.

Для доказательства достаточно для конечного $C_{i}$ допустить, что

$$
g_{i}:\left(A \cup C_{i}\right) \rightarrow\left(A^{\prime} \cup C_{i}^{\prime}\right)
$$

продолжает $g$ и является $L$-элементарным (( $L, P)$-элементарным) отображением, выбрать произвольное $c \in V$ и найти такой $c^{\prime} \in V$, что, если положить

$$
g_{i+1}=g_{i} \cup\left\{\left(c, c^{\prime}\right)\right\},
$$

отображение $g_{i+1}$ окажется $L$-элементарным (соответственно окажется $(L, P)$ элементарным) отображением.

Но существование такого $c^{\prime}$ следует из определения псевдоконечной однородности, из того, что активная область каждого псевдоконечного состояния является псевдоконечным множеством, и из того, что обогащение $\omega$-насыщенной структуры конечным множеством выделенных элементов оставляет структуру $\omega$-насыщенной.

Докажем, что активная область каждого псевдоконечного состояния является псевдоконечным множеством (это замечено в доказательстве теоремы 5.4 из [3]).

Рассмотрим схему $\tau=\{P\}$ базы данных, в которой $P$ является одноместным символом отношения. Для любого $(L, \tau)$-высказывания $\gamma$ и любого $\rho$-состояния $s$ легко построить $(L, \rho)$-высказывание $\gamma^{*}$, для которого $(V, s)=\gamma^{*}$ тогда и только тогда, когда $(V, \mathrm{AD}(s)) \models \gamma$.

Пусть теперь $s$ является псевдоконечным $\rho$-состоянием в $V$ и $\gamma \in \operatorname{Fin}(V, \tau)$. Так как активная область любого конечного состояния конечна, то $(V, r) \models \gamma^{*}$ для любого конечного $\rho$-состояния $r$. Поэтому $(V, s) \models \gamma^{*}$ и, значит, $(V, \operatorname{AD}(s)) \models \gamma$.

ОПРЕДЕЛЕНИЕ 19. Полная теория $T$ обладает первым свойством изолированности, если существуют модель $M$ полной теории $T$ и бесконечное множество $I$, которое является неразличимой последовательностью в $M$, такие, что

для любых специальной структуры $(N, J)$, элементарно эквивалентной структуре $(M, I)$, псевдоконечного подмножества $A$ множества $J$ в $N$, конечного подмножества $C$ модели $N$ и элемента $a$ модели $N$ существует такое счетное $A_{0} \subseteq A$, что

$$
\operatorname{tp}\left(a /\left(A_{0} \cup C\right)\right)
$$

изолирует $\operatorname{tp}(a /(A \cup C))$ в $N$. 
В этом случае мы также говорим, что $(M, I)$ обладает первым свойством изолированности.

ОПРЕДЕЛЕНИЕ 20. Полная теория Т обладает вторым свойством изолированности, если существуют модель $M$ полной теории $T$ и бесконечное множество $I$, которое является неразличимой последовательностью в $(M, I)$, такие, чTо

для любых специальной структуры $(N, J)$, элементарно эквивалентной структуре $(M, I)$, псевдоконечного подмножества $A$ множества $J$ в $N$, конечного подмножества $C$ модели $N$ и элемента $a$ модели $N$ существует такое счетное $A_{0} \subseteq A$, что

$$
\operatorname{tp}\left(a /\left(A_{0} \cup C\right)\right)
$$

изолирует $\operatorname{tp}(a /(A \cup C))$ в $(N, J)$.

В этом случае мы также говорим, что $(M, I)$ обладает вторым свойством изолированности.

ТЕОрема 6.4. Если теория Т обладает первым (соответственно вторым) свойством изолированности, то она обладает первым (соответственно вторъмм свойством псевдоконечной однородности.

ДокаЗАТЕЛЬСтво. Пусть $M$ является моделью теории $T$. Пусть $(N, J) \equiv$ $(M, I), A$ и $B$ являются псевдоконечными подмножествами множества $J$ в модели $N, C$ и $D$ являются конечными подмножествами модели $N$, отображение $h:(A \cup C) \rightarrow(B \cup D)$ является элементарным отображением в $N$ (соответственно в $(N, J))$ с $\omega$-насыщенной $(N, A, B, h)$ (соответственно $(N, J, A, B, h))$ и $a \in N$.

Найдем такой элемент $b \in N$, что $h \cup\{(a, b)\}$ является элементарным отображением в $N$ (соответственно в $(N, J))$.

Рассмотрим специальную модель $\left(N_{1}, J_{1}\right)$, являющуюся элементарным расширением

$$
(N, J)
$$

такой несчетной мощности $\kappa=\kappa^{*}$, что $\operatorname{cf}(\kappa)>\omega$. Существует такое счетное $A_{0} \subseteq A$, что

$$
p_{0}=\operatorname{tp}\left(a /\left(A_{0} \cup C\right)\right)
$$

изолирует $p=\operatorname{tp}(a /(A \cup C))$ в $N_{1}$ (соответственно в $\left.\left(N_{1}, J_{1}\right)\right)$. Так как $h$ является элементарным отображением, то $h(p)$ является типом над $(B \cup D)$ и $h\left(p_{0}\right)$ изолирует $h(p)$. Как обычно, для множества $q$ формул сигнатуры $L(A \cup C)$, не содержащих свободных переменных, отличных от $x$, через $h(q)$ мы обозначаем множество формул $\{\theta(x, h(\bar{c})) \mid \theta(x, \bar{c}) \in q\}$. Другими словами, в каждой формуле каждую константу $c \in(A \cup C)$ заменяем на константу $h(c)$. Так как $\operatorname{cf}(\kappa)>\omega$, то система $\left(N_{1}, J_{1}\right)$ является $\omega^{+}$-насыщенной. Следовательно, существует $b_{1} \in N_{1}$, реализующий $h\left(p_{0}\right)$ и, значит, $h(p)$. Поэтому $h \cup\left\{\left(a, b_{1}\right)\right\}$ является элементарным отображением в $N_{1}$ (соответственно в $\left(N_{1}, J_{1}\right)$ ). Но так как $(N, A, B, h)$ (соответственно $(N, J, A, B, h)$ ) является $\omega$-насыщенной, то найдется и такой $b \in N$, что $h \cup\{(a, b)\}$ является элементарным отображением в $N$ (соответственно в $(N, J))$. Теорема 6.4 доказана. 
Формулы сигнатуры $\{<\}$ называются порядковыми.

ОПРедЕЛЕНиЕ 21. Выражение $(\exists x \in P) \Psi$ является сокращением для

$$
(\exists x)(P(x) \wedge \Psi)
$$

а выражение $(\forall x \in P) \Psi$ является сокращением для

$$
(\forall x)(P(x) \rightarrow \Psi)
$$

Пусть $K$ - некоторая совокупность $(L, P)$-формул. $(L, P)$-структура $(M, I)$ называется $(P, K)$-сводимой, если

для каждой формулы $\phi(\bar{x}, \bar{y})$ из $K$ существует такая сводящая бескванторная порядковая формула $\psi(\bar{w}, \bar{y})$, что для каждой последовательности $\bar{m}$ элементов $M$ существует последовательность $\bar{c}_{\bar{m}} \in I$, для которой

$$
(\forall \bar{y} \in P)\left(\psi\left(\bar{c}_{\bar{m}}, \bar{y}\right) \leftrightarrow \phi(\bar{m}, \bar{y})\right)
$$

$(L, P)$-структура $(M, I)$ называется эффективно $(P, K)$-сводимой, если существует алгоритм, который

по каждой формуле $\phi(\bar{x}, \bar{y})$ из $K$ строит такую сводящую бескванторную порядковую формулу $\psi(\bar{w}, \bar{y})$, что для каждой последовательности $\bar{m}$ элементов $M$ существует последовательность $\bar{c}_{\bar{m}} \in I$, для которой

$$
(\forall \bar{y} \in P)\left(\psi\left(\bar{c}_{\bar{m}}, \bar{y}\right) \leftrightarrow \phi(\bar{m}, \bar{y})\right)
$$

Если $K$-это множество всех $L$-формул, то $(P, K)$-сводимость называется $P$ сводимостъю. Если же $K$ состоит из всех $(L, P)$-формул, то $(P, K)$-сводимость называется далее сильной $P$-сводимостью.

ОПРеДЕЛЕниЕ 22. ( $L, P)$-формула называется $P$-ограниченной ${ }^{4}$, если она не содержит $P$ или имеет вид $(\forall x \in P) \Psi$ или $(\exists x \in P) \Psi$, где $\Psi$ является $P$-ограниченной формулой. Квантор $(\forall x \in P)$ называется ограниченным квантором всеобщности, а квантор $(\exists x \in P)$ называется ограниченным квантором существования.

$(L, P)$-структуру $(M, I)$ называют $P$-ограниченной, если каждая $(L, P)$-формула эквивалентна в $(M, I)$ некоторой $P$-ограниченной формуле.

ЗАМЕЧАНИЕ 6.5. Пусть $(I,<)$ - плотный линейный порядок без концевых элементов. Каждая порядковая формула $\psi(\bar{y})$ эквивалентна на $(I,<)$ бескванторной порядковой формуле.

ДоказАТЕльство. Формула $(\exists x)(y<x<z)$ эквивалентна формуле $y<z$.

Лемма 6.6 (см. [5] и [15; теорема 2.5]). Каждая Р-сводимая и Р-ограниченная $(L, P)$-структура $(M, I)$, в которой I плотно упорядоченно без концевых элементов, является сильно Р-сводимой.

\footnotetext{
${ }^{4}$ Англ. P-bounded.
} 
ДоказАтельство. Допустим, что для $\phi(\bar{x}, \bar{y}, z)$ существует такая бескванторная порядковая формула $\psi(\bar{w}, \bar{y}, z)$, что для каждой последовательности $\bar{m}$ найдется последовательность $\bar{c}_{\bar{m}} \in I$, для которой

$$
(\forall \bar{y} \in P)(\forall z \in P)\left(\psi\left(\bar{c}_{\bar{m}}, \bar{y}, z\right) \leftrightarrow \phi(\bar{m}, \bar{y}, z)\right) .
$$

Тогда

$$
(\forall \bar{y} \in P)\left((\forall z \in P) \psi\left(\bar{c}_{\bar{m}}, \bar{y}, z\right) \leftrightarrow(\forall z \in P) \phi(\bar{m}, \bar{y}, z)\right) .
$$

Из того, что $I$ плотно упорядоченно без концевых элементов, следует, что формула

$$
(\forall z \in P) \psi(\bar{w}, \bar{y}, z)
$$

эквивалентна в $(I,<)$ бескванторной формуле.

ТеОрема 6.7. Пусть $M$ - модель полной $L$-теории $T$ и бесконечное множество I является неразличимой плотно упорядоченной без концевых точек последовательностью в $M$.

Пусть $(M, I)$ является $P$-сводимой и $P$-ограниченной. Тогда $I$ является неразличимой последовательностью в $(M, I)$ и Т обладает вторым свойством изолированности.

ДокАзАтельство. Прежде всего заметим, что $I$ является неразличимой последовательностью в $(M, I)$.

Действительно, $I$ не различается бескванторными $(L, P)$-формулами и $L$ формулами. Допустим, что $I$ не различается $P$-ограниченными формулами с числом $P$-кванторов, не превосходящим $n$, и рассмотрим формулу $(\exists x \in P) \Psi$. Понятно, что если $\Psi$ выполняется на первом наборе при некотором значении $x$, то $\Psi$ выполняется и на так же упорядоченном втором наборе при таком значении $x$, относительное расположение которого относительно второго набора аналогично расположению первого значения $x$ относительно первого набора. Рассмотрение формулы $(\forall x \in P) \Psi$ сводится к тому, что для каждого значения $x$ можно найти другое значение $x$, которое расположено относительно первого набора аналогично тому, как первое значение $x$ расположено относительно второго набора.

Теперь докажем изолированность. Рассмотрим произвольное псевдоконечное множество $A \subseteq I$ и конечное множество $C$. Возьмем произвольные элемент $a \in M$ и конечную последовательность элементов $\bar{m}$ в $C$. Для каждой $(L, P)$-формулы $\phi(z, \bar{x}, \bar{y})$ найдется такая бескванторная порядковая формула $\psi_{\phi}(\bar{w}, \bar{y})$, что найдется последовательность $\bar{c}_{\bar{m}, a} \in I$, для которой

$$
(\forall \bar{y} \in P)\left(\psi_{\phi}\left(\bar{c}_{\bar{m}, a}, \bar{y}\right) \leftrightarrow \phi(a, \bar{m}, \bar{y})\right) .
$$

Для каждой $\phi(z, \bar{x}, \bar{y})$ зафиксируем одну последовательность $\bar{c}_{\bar{m}, a}$, для которой имеет место (4). Из псевдоконечности $A$ следует, что для каждого элемента $b$ последовательности $\bar{c}_{\bar{m}, a}$ либо этот элемент лежит в $A$ и мы включаем $b$ в $A_{\phi}$, либо этот элемент больше всех элементов $A$ и мы включаем наибольший элемент $A$ в $A_{\phi}$, либо этот элемент меньше всех элементов $A$ и мы включаем наименьший элемент $A$ в $A_{\phi}$, либо существуют самый больший элемент ${ }_{b} a$ в $A$ из меньших $b$ и самый меньший элемент $a_{b}$ в $A$ из больших $b$ и мы включаем оба 
эти крайние элементы $b a$ и $a_{b}$ в $A_{\phi}$. Ясно, что порядковый бескванторный тип последовательности $\bar{d} \in A$ над $\bar{c}_{\bar{m}, a}$ определяется порядковым бескванторным типом этой последовательности $\bar{d} \in A$ над $A_{\phi}$. Объединение $A_{0}$ всех $A_{\phi}$ для всех $\phi$ счетно. Понятно, что тип $a$ над $A_{0}$ в $(M, I, \bar{m})$ изолирует тип $a$ над $A$ в $(M, I, \bar{m})$.

\section{7. Сводимые теории}

ОПРЕДЕЛЕНИЕ 23 (сводимой теории). Универсум $U$ конечной сигнатуры $L$ называется сводимым, если существует такая $P$-сводимая $(L, P)$-структура $(M, I)$, что $M \equiv U$, а $I$ является бесконечной неразличимой последовательностью в $M$. Для сводимого универсума $U$ теория $\operatorname{Th}(U)$ тоже называется сводимой. Сводимый универсум $U$ и его теория называются эффективно сводимыми, если существует такая эффективно $P$-сводимая $(L, P)$-структура $(M, I)$, что $M \equiv U$, а $I$ является бесконечной неразличимой последовательностью в $M$.

ТЕОРема 7.1. Для каждого сводимого универсума $U$ конечной сигнатуpы $L$ существует такая $P$-сводимая $(L, P)$-структура $(N, J)$, что $N \equiv U, J$ является неразличимой последовательностью в $N$, ограничение < на J задает на $J$ такой линейный порядок, что $(J,<)$ является упорядоченным обычным образом множеством действительных чисел, а $N$ является $\left(2^{\omega}\right)^{+}$-насыщенной системой.

Доказательство. Рассмотрим произвольную $P$-сводимую $(L, P)$-структуру $(M, I)$, для которой $M \equiv U$, а $I$ является бесконечной неразличимой последовательностью в $M$. Выберем такой несчетный кардинал $\kappa$, больший мощности $M$, что $\operatorname{cf}(\kappa)>2^{\omega}$ и $\kappa=\kappa^{*}$. Рассмотрим специальное элементарное расширение $(V, J)$ мощности $\kappa(L, P)$-структуры $(M, I)$. Из условия $\operatorname{cf}(\kappa)>2^{\omega}$ следует, что $(V, J)$ является $\left(2^{\omega}\right)^{+}$-насыщенной системой. Из определения $\left(2^{\omega}\right)^{+}$насыщенности следует, что можно считать, что $(J,<)$ является расширением упорядоченного обычным образом множества $\mathbb{R}$ действительных чисел. Так как $(V, J) \succeq(M, I)$, то $(V, J)$ тоже является $P$-сводимой $(L, P)$-структурой.

Остается доказать, что $(V, \mathbb{R})$ является $P$-сводимой $(L, P)$-структурой.

Рассмотрим произвольную $L$-формулу $\phi(\bar{x}, \bar{y})$. Для этой формулы $\phi(\bar{x}, \bar{y})$ существует такая бескванторная порядковая формула $\psi(\bar{w}, \bar{y})$, что для каждой последовательности $\bar{m}$ элементов $V$ существует последовательность $\bar{c}_{\bar{m}} \in J$, для которой

$$
(\forall \bar{y} \in P)\left(\psi\left(\bar{c}_{\bar{m}}, \bar{y}\right) \leftrightarrow \phi(\bar{m}, \bar{y})\right)
$$

выполняется в $(V, J)$. Можно считать, что $\psi(\bar{w}, \bar{y})$ является дизъюнкцией конъюнкций формул вида $u<v$ и $u=v$, в которых $u$ и $v$ - это элементы кортежей $\bar{w}$ и $\bar{y}$.

Наша цель - найти такую порядковую формулу

$$
\theta(\bar{w}, \bar{u}, \bar{z}, \bar{y})
$$


что для каждой последовательности $\bar{m}$ элементов $V$ существуют такие последовательности $\bar{a}_{\bar{m}} \in \mathbb{R}, \bar{d}_{\bar{m}} \in \mathbb{R}$ и $\bar{e}_{\bar{m}} \in \mathbb{R}$, для которых

$$
(\forall \bar{y} \in P)\left(\theta\left(\bar{a}_{\bar{m}}, \bar{d}_{\bar{m}}, \bar{e}_{\bar{m}}, \bar{y}\right) \leftrightarrow \phi(\bar{m}, \bar{y})\right)
$$

выполняется в $(V, \mathbb{R})$.

Кортеж переменных $\bar{u}$ представляет собой кортеж двойников для переменных из кортежа $\bar{w}$. Кортежи $\bar{w}, \bar{u}$ и $\bar{y}$ попарно не содержат общих переменных. Кортеж переменных $\bar{z}$ определим позже.

Рассмотрим произвольную последовательности $\bar{m}$ элементов $V$ и последовательность $\bar{c}_{\bar{m}} \in J$.

Рассмотрим произвольное сохраняющее порядок отображение элементов последовательности $\bar{c}_{\bar{m}}$ в $\mathbb{R}$. Пусть при этом отображении последовательность $\bar{c}_{\bar{m}}$ перейдет в последовательность $\bar{a}_{\bar{m}} \in \mathbb{R}$. Таким образом мы выберем значения $\bar{a}_{\bar{m}}$ для переменных $\bar{w}$.

Рассмотрим произвольный элемент $c$ из последовательности $\bar{c}_{\bar{m}}$, являющийся значением переменной $w_{i}$, и найдем значение $d(c)$ для двойника $u_{i}$ этой переменной.

Если $c \in \mathbb{R}$, то пусть $d(c)=c$. Если $c$ больше всех элементов $\mathbb{R}$, то пусть $d(c)=\infty$. Если $c$ меньше всех элементов $\mathbb{R}$, то пусть $d(c)=-\infty$.

Если же ни один из предыдущих случаев не выполняется, то $c$ разбивает все действительные числа на непустой класс меньших $c$ действительных чисел и непустой класс больших $c$ действительных чисел. В качестве $d(c)$ в этом случае возьмем либо наибольшее число в первом классе, либо наименьшее число во втором.

Мы будем использовать последовательность $d\left(\bar{c}_{\bar{m}}\right)$ как последовательность значений для последовательности $\bar{u}$ переменных.

Формулу $\psi(\bar{w}, \bar{y})$ исправим следующим образом. В каждом равенстве и неравенстве, содержащем переменную из кортежа $\bar{y}$, заменим каждую переменную из кортежа $\bar{w}$ на двойник этой переменной из кортежа $\bar{u}$. Получим формулу

$$
\theta^{*}(\bar{w}, \bar{u}, \bar{y})
$$

Значение $c$ переменной $w_{i}$ из $\bar{w}$ либо входит в $\mathbb{R}$, либо больше всех элементов $\mathbb{R}$, либо меньше всех элементов $\mathbb{R}$, либо разбивает все действительные числа на непустой класс меньших $c$ действительных чисел и непустой класс больших $c$ действительных чисел. В последнем случае либо в первом классе есть наибольшее число, либо во втором классе есть наименьшее число.

Для каждой переменной $w_{i}$ из $\bar{w}$, которая встречается в $\psi(\bar{w}, \bar{y})$ в равенствах и неравенствах с переменными из $\bar{y}$, мы рассматриваем все эти пять случаев. Если имеется $k$ таких переменных, всего рассматриваем $5^{k}$ случаев.

Равенства и неравенства с переменными из $\bar{y}$ в каждом из рассматриваемых $5^{k}$ случаев исправляем в формуле

$$
\theta^{*}(\bar{w}, \bar{u}, \bar{y})
$$

следующим образом.

Если значение $c$ переменной $w_{i}$ из $\bar{w}$ входит в $\mathbb{R}$, равенства и неравенства с $u_{i}$ не исправляются. 
Если значение $c$ переменной $w_{i}$ из $\bar{w}$ больше всех элементов $\mathbb{R}$, неравенства $y_{j}<u_{i}$ считаем истинными и заменяем на $y_{j}=y_{j}$, а неравенства $u_{i}<y_{j}$ и равенства $y_{j}=u_{i}$ и $u_{i}=y_{j}$ считаем ложными и заменяем на $y_{j}<y_{j}$.

Если значение $c$ переменной $w_{i}$ из $\bar{w}$ меньше всех элементов $\mathbb{R}$, неравенства $u_{i}<y_{j}$ считаем истинными и заменяем на $y_{j}=y_{j}$, а неравенства $y_{j}<u_{i}$ и равенства $u_{i}=y_{j}$ и $y_{j}=u_{i}$ считаем ложными и заменяем на $y_{j}<y_{j}$.

Если значение $c$ переменной $w_{i}$ из $\bar{w}$ не входит в $\mathbb{R}$ и разбивает все действительные числа на непустой класс меньших $c$ действительных чисел и непустой класс больших $c$ действительных чисел и в первом классе есть наибольшее число, каждое неравенство $y_{j}<u_{i}$ заменяем на

$$
\left(y_{j}<u_{i} \vee y_{j}=u_{i}\right)
$$

Равенства $y_{j}=u_{i}$ и $u_{i}=y_{j}$ в этом случае считаем ложными и заменяем на $y_{j}<y_{j}$.

Если значение $c$ переменной $w_{i}$ из $\bar{w}$ не входит в $\mathbb{R}$ и разбивает все действительные числа на непустой класс меньших $c$ действительных чисел и непустой класс больших $c$ действительных чисел и во втором классе есть наименьшее число, каждое неравенство $u_{i}<y_{j}$ заменяем на

$$
\left(u_{i}<y_{j} \vee y_{j}=u_{i}\right)
$$

Равенства $y_{j}=u_{i}$ и $u_{i}=y_{j}$ в этом случае считаем ложными и заменяем на $y_{j}<y_{j}$.

Для $i$-го случая получаем формулу $\theta_{i}(\bar{w}, \bar{u}, \bar{y})$.

Пусть $z_{0}, z_{1}, \ldots, z_{5^{k}}$ являются попарно различными переменными, не входящими ни в один из кортежей $\bar{u}, \bar{y}$ и $\bar{w}$. Пусть

$$
\bar{z}=z_{0}, z_{1}, \ldots, z_{5^{k}}
$$

Понятно, что в качестве

$$
\theta(\bar{w}, \bar{u}, \bar{z}, \bar{y})
$$

можно взять

$$
\left(z_{1}<\cdots<z_{5^{k}} \wedge \bigvee_{i=1}^{5^{k}}\left(\theta_{i}(\bar{w}, \bar{u}, \bar{y}) \wedge z_{0}=z_{i}\right)\right)
$$

ОПРЕДЕЛЕНИЕ 24 (независимой формулы [19], [20], [5]). Пусть $M-L$-структура. $L$-формула $\phi(\bar{x}, \bar{y})$ называется независимой в $M$, если для каждого натурального $n$ найдутся такие наборы значений

$$
\bar{a}_{1}, \ldots, \bar{a}_{n}
$$

для набора переменных $\bar{x}$, что выполнено условие

(А) для любого $\eta \subseteq\{1, \ldots, n\}$ найдется такой набор значений $\bar{b}_{\eta}$ для набора переменных $\bar{y}$, что

$$
\eta=\left\{i \in\{1, \ldots, n\} \mid M \models \phi\left(\bar{a}_{i}, \bar{b}_{\eta}\right)\right\} .
$$


Теорема 7.2 [5; теорема 5.2]. Пусть бесконечное множество I является неразличимой плотно полно упорядоченной без концевых элементов последовательностью в универсуме $M$ сигнатурь $L$.

Если $M$ не имеет никакой независимой формуль, то $(M, I)$ является $P$ сводимой.

ДоказАтЕЛЬство. Рассмотрим произвольную $L$-формулу $\phi(\bar{x}, \bar{y})$.

Так как эта формула $\phi(\bar{x}, \bar{y})$ не является независимой в $M$, то найдется такое $n$, для которого любые наборы значений

$$
\bar{a}_{1}, \ldots, \bar{a}_{n}
$$

для набора переменных $\bar{x}$ из носителя структуры $M$ и, тем более, из $I$ не удовлетворяют условию (А).

Пусть $\bar{x}=x_{1}, \ldots, x_{m}$. Пусть $\bar{x}_{i}=x_{i, 1}, \ldots, x_{i, m}$ для $i=1,2$.

Порядковым бескванторным типом $p\left(\bar{x}_{1}, \bar{x}_{2}\right)$ от переменных $\left(\bar{x}_{1}, \bar{x}_{2}\right)$ над пустым множеством называется такое совместное множество формул видов $x_{i, j}<x_{k, l}$ и $x_{i, j}=x_{k, l}$, где $i, k \in\{1,2\}$ и $j, l \in\{1, \ldots, m\}$, которое не содержится ни в каком отличном от него совместном множестве таких формул. Тип $p\left(\bar{x}_{1}, \bar{x}_{2}\right)$ реализуется набором $\left(\bar{a}_{i}, \bar{a}_{j}\right)$ элементов множества $I$, если все формулы из $p\left(\bar{a}_{i}, \bar{a}_{j}\right)$ истинны, другими словами, при замене $x_{1, k}$ на $a_{i, k}$ и $x_{2, k}$ на $a_{j, k}$ все формулы из $p\left(\bar{x}_{1}, \bar{x}_{2}\right)$ станут истинными.

Понятно, что для заданного натурального положительного числа $m$ можно найти такое натуральное положительное число $\tau(m)$, что существует точно $\tau(m)$ различных порядковых бескванторных типов над пустым множеством от фиксированного набора, составленного из $2 m$ переменных. Поэтому, по теореме Рамсея о конечных множествах, в любой последовательности

$$
\bar{a}_{1}, \ldots, \bar{a}_{r(2, \tau(m), n+1)}
$$

найдется подпоследовательность длины $n+1$, любая пара членов которой, в которой первый элемент предшествует в последовательности второму элементу этой пары, реализует один и тот же порядковый бескванторный тип над пустым множеством. Пусть $r=r(2, \tau(m), n+1)$.

Порядковым бескванторным типом $p(\bar{x}, C)$ от переменных $\bar{x}=x_{1}, \ldots, x_{m}$ над заданным конечным множеством $C \subseteq I$ называется такое совместное множество формул видов $x_{j}<x_{l}, x_{j}=x_{l}, x_{j}<c, c<x_{j}$ и $x_{j}=c$, где $j, l \in\{1, \ldots, m\}$, a $c \in C$, которое не содержится ни в каком отличном от него совместном множестве таких формул. Тип $p(\bar{x}, C)$ реализуется набором $\bar{a}$ элементов множества $I$, если все формулы из $p(\bar{a}, C)$ истинны, другими словами, при замене $x_{j}$ на $a_{j}$ для $j=1, \ldots, n$ все формулы из $p(\bar{x}, C)$ станут истинными.

Пусть $c_{1}, d_{1}, \ldots, c_{m}, d_{m}$ являются элементами множества $I$.

Окрестностью $\left(c_{1}, d_{1}\right), \ldots,\left(c_{m}, d_{m}\right)$ такого набора $\bar{a}$ в $I^{m}$, что $c_{1}<a_{1}<d_{1}$, $\ldots, c_{m}<a_{m}<d_{m}$, называется множество всех таких наборов $\bar{b}$ элементов множества $I$, что $c_{1}<b_{1}<d_{1}, \ldots, c_{m}<b_{m}<d_{m}$.

Окрестностью набора $\bar{a}$ в типе $p(\bar{x}, C)$, реализуемом набором $\bar{a}$, называется пересечение окрестности этого набора $\bar{a}$ в $I^{m}$ с множеством всех наборов, реализующих тип $p(\bar{x}, C)$. 
Зафиксируем набор $\bar{b}$ элементов носителя структуры $M$ той же длины, что и набор переменных $\bar{y}$. Пусть

$$
V_{\bar{b}}=\{\bar{d} \in I \mid M \models \phi(\bar{d}, \bar{b})\} .
$$

Последовательность

$$
\bar{a}_{1}, \ldots, \bar{a}_{i}
$$

наборов элементов множества $I$ называется согласованной ${ }^{5}$, если для каждого $j \in\{1, \ldots, i\}$ в каждой окрестности $\bar{a}_{j}$ в типе $p\left(\bar{x}, C_{j}\right)$, реализуемом набором $\bar{a}_{j}$, найдутся как наборы, лежащие в $V_{\bar{b}}$, так и наборы, не лежащие в $V_{\bar{b}}$. Здесь $C_{j}$ обозначает множество всех элементов, входящих хотя бы в один набор из последовательности

$$
\bar{a}_{1}, \ldots, \bar{a}_{j-1} .
$$

При $j=1$ множество $C_{j}$ пусто.

Согласованная последовательность

$$
\bar{a}_{1}, \ldots, \bar{a}_{i}
$$

называется покрытием, если ее нельзя продолжить более длинной согласованной последовательностью.

ЛЕмма 7.3. Не существует никакой согласованной последовательности длины $r$.

ДОКАЗАТЕЛЬСТВо ЛЕММЫ. Из согласованной последовательности длины $r$ можно выделить такую подпоследовательность

$$
\bar{d}_{0}, \bar{d}_{1}, \ldots, \bar{d}_{n}
$$

длины $n+1$, любая пара членов которой, в которой первый элемент предшествует в последовательности второму элементу этой пары, реализует один и тот же порядковый бескванторный тип над пустым множеством.

Сохраним набор $\bar{d}_{0}$. Остальные наборы исправим следующим образом. Если в этой подпоследовательности какой-то элемент следующего набора равен какому-то элементу предыдущего набора, то найдется такое место, что в любых двух наборах этой подпоследовательности элементы, стоящие на этом месте, равны. В этом случае мы это место удалим из каждого набора рассматриваемой подпоследовательности. После этого каждый элемент следующего набора отличен от каждого элемента предыдущего набора. Если же в каком-то наборе этой подпоследовательности на каких-то двух разных местах стоят одинаковые элементы, то в любом наборе из этой подпоследовательности на этих местах находятся одинаковые элементы. Элементы набора $\bar{d}_{0}$ и оставленные элементы всех других наборов рассматриваемой подпоследовательности расположим в порядке возрастания. Для элементов $u_{i}$ и $u_{i-1}$, расположенных на $i$-м и $(i-1)$-м местах, выберем $v_{i} \in I$ так, чтобы $u_{i-1}<v_{i}<u_{i}$. Пусть еще $v_{1}<u_{1}$

\footnotetext{
${ }^{5}$ Англ. coherent.
} 
и $u_{k}<v_{k+1}$ для самого большого оставленного элемента $u_{k}$. Будем рассматривать окрестности $\left(v_{i}, v_{i+1}\right)$ элементов $u_{i}$, а окрестностью набора из подпоследовательности будем называть набор, составленный из выброшенных элементов рассматриваемого набора и окрестностей оставленных элементов этого набора.

Рассмотрим произвольное $\eta \subseteq\{1, \ldots, n\}$. Если $i \in \eta$, выберем в рассматриваемой окрестности набора $\bar{d}_{i}$ набор $\bar{c}_{i}$, лежащий в $V_{\bar{b}}$. Если же $i \notin \eta$, выберем в рассматриваемой окрестности набора $\bar{d}_{i}$ набор $\bar{c}_{i}$, не лежащий в $V_{\bar{b}}$. По построению, последовательности

$$
\bar{d}_{1}, \ldots, \bar{d}_{n}
$$

и

$$
\bar{c}_{1}, \ldots, \bar{c}_{n}
$$

одинаково упорядочены. Из неразличимости $I$ следует, что существует такой набор $\bar{b}_{\eta}$, что

$$
\eta=\left\{i \in\{1, \ldots, n\} \mid M \models \phi\left(\bar{d}_{i}, \bar{b}_{\eta}\right)\right\} .
$$

Но это противоречит выбору $n$. Лемма 7.3 доказана.

Из леммы следует, что каждая согласованная последовательность продолжается до покрытия. Пусть

$$
\bar{a}_{1}, \ldots, \bar{a}_{i}
$$

является покрытием. Это означает, что для любого $\bar{a}_{i+1} \in I$ существует окрестность этого набора $\bar{a}_{i+1}$ в типе $p\left(\bar{x}, C_{i+1}\right)$, реализуемом набором $\bar{a}_{i+1}$, содержащая или только наборы, лежащие в $V_{\bar{b}}$, или только наборы, не лежащие в $V_{\bar{b}}$.

ЛЕмма 7.4. Пусть $D \subseteq I$ удовлетворяет условию:

для любого $\bar{a}_{i+1} \in I$ существует окрестность этого набора $\bar{a}_{i+1}$ в типе $p(\bar{x}, D)$, реализуемом набором $\bar{a}_{i+1}$, содержащая или только наборы первого сорта, или только наборы второго сорта.

Для каждого типа $p(\bar{x}, D)$ либо все наборы $\bar{a}_{i+1}$, реализующие этот тuп, имеют первый сорт, либо все наборы $\bar{a}_{i+1}$, реализующие этот тип, имеют второй сорт.

ДоказАтельство ЛЕммы. Ради краткости будем говорить, что набор лежит в типе, если он реализует этот тип. Индукция по $m$. При $m=1$ тип $p(x, D)$ задается либо условием $x=c$, либо условием $x<c$, либо условием $c<x$, либо условием $c<x<d$. Рассмотрим только последний случай, так как в первом утверждение выполняется очевидным образом, а в других двух доказательство аналогично. Пусть в этом типе содержатся как элементы первого сорта, так и элементы второго сорта. Выберем произвольные элементы $a$ из этого типа первого сорта и $е$ из этого типа второго сорта. Будем считать, для определенности, что $a<e$. Другой случай рассматривается аналогично. Отнесем элемент $a^{\prime}$ из этого типа к первой части, если $a^{\prime}$ не превосходит $a$ или если весь интервал $\left(a, a^{\prime}\right)$ содержит только элементы первого сорта. Остальные элементы этого типа отнесем ко второй части. Ясно, что каждый элемент первой части меньше каждого элемента второй. Обе части непусты. Из полноты порядка на $I$ следует, что либо в первой части есть наибольший элемент, либо 
во второй части есть наименьший элемент. Если таковым является $a_{1}$, то рассмотрим окрестность $a_{1}$, в которой либо все элементы первого сорта, либо все элементы второго сорта. Второй случай невозможен, а первый противоречит выбору $a_{1}$.

Пусть для $m-1$ утверждение доказано. С типом $p(\bar{x}, D)$ свяжем тип

$$
p^{\prime}\left(x_{1}, \ldots, x_{m-1}, D\right)
$$

полученный удалением формул, в которых встречается $x_{m}$. Для каждого набора $a_{1}, \ldots, a_{m-1}$, реализующего $p^{\prime}\left(x_{1}, \ldots, x_{m-1}, D\right)$, пусть $A$ состоит из всех элементов набора $a_{1}, \ldots, a_{m-1}$. Пусть

$$
p^{\prime \prime}\left(x_{m}, D \cup A\right)
$$

обозначает тип, получаемый из $p(\bar{x}, D)$ заменой $x_{1}, \ldots, x_{m-1}$ на $a_{1}, \ldots, a_{m-1}$. Легко заметить, что каждый элемент $a_{m}$ из $p^{\prime \prime}\left(x_{m}, D \cup A\right)$ имеет окрестность, все элементы которой вместе с $a_{1}, \ldots, a_{m-1}$ либо первого сорта, либо второго сорта. По предыдущему, либо все элементы $p^{\prime \prime}\left(x_{m}, D \cup A\right)$ вместе с $a_{1}, \ldots, a_{m-1}$ первого сорта, либо все второго сорта. Отнесем $a_{1}, \ldots, a_{m-1}$ к первому сорту, если все элементы $p^{\prime \prime}\left(x_{m}, D \cup A\right)$ вместе с $a_{1}, \ldots, a_{m-1}$ первого сорта, и ко второму сорту, если все элементы $p^{\prime \prime}\left(x_{m}, D \cup A\right)$ вместе с $a_{1}, \ldots, a_{m-1}$ второго сорта. Каждый набор $a_{1}, \ldots, a_{m-1}$ из

$$
p^{\prime}\left(x_{1}, \ldots, x_{m-1}, D\right)
$$

имеет окрестность, в которой все наборы имеют один и тот же сорт. По индукции, все наборы из $p^{\prime}\left(x_{1}, \ldots, x_{m-1}, D\right)$ имеют один сорт. Лемма 7.4 доказана.

Таким образом, каждый тип $p\left(\bar{x}, C_{i+1}\right)$ состоит либо только из наборов, лежащих в $V_{\bar{b}}$, либо только из наборов, не лежащих в $V_{\bar{b}}$. Это означает, что $V_{\bar{b}}$ является объединением нескольких типов.

Пусть $C_{i+1}$ содержит $k$ элементов. Каждому типу поставим в соответствие конъюнкцию всех формул, входящих в этот тип, в которых элементы из $C_{i+1}$ заменены на переменные $z_{1}, \ldots, z_{k}$, и рассмотрим всевозможные дизъюнкции полученных формул. Пусть имеется $l$ дизъюнкций $\Psi_{1}, \ldots, \Psi_{l}$. Пусть, наконец, набор $\bar{z}$ содержит $k+l+1$ переменную. В качестве сводящей для $\phi(\bar{x}, \bar{y})$ годится порядковая формула

$$
\bigvee_{j=1}^{l}\left(z_{k+l+1}=z_{k+j} \wedge \Psi_{j}\right) .
$$

Теорема 7.2 доказана.

В заключение приведем еще один результат, показывающий, что свойства сводимости и относительной изолированности в определенном смысле эквивалентны.

Скажем, что наборы элементов $\bar{d}_{1}=\left(d_{1}^{1}, \ldots, d_{1}^{m}\right)$ и $\bar{d}_{2}=\left(d_{2}^{1}, \ldots, d_{2}^{m}\right)$ из неразличимой последовательности $I$ в $L$-структуре $M$, имеющие ту же длину, что и набор переменных $\bar{x}$, являются различающей парой для $L$-формулы $\phi(\bar{x}, \bar{y})$ и набора $\bar{a}$ элементов из $M$, длина которого совпадает с длиной набора переменных $\bar{y}$, если: 
1) элементы этих наборов одинаково упорядочены, другими словами, для любых $1 \leqslant i, j \leqslant m$ тогда и только тогда $d_{1}^{i}<d_{1}^{j}$, когда $d_{2}^{i}<d_{2}^{j}$;

2) существует такое $i_{0}$, что $d_{1}^{i}=d_{2}^{i}$ для всех $i$ из $\{1, \ldots, m\}$, отличных от $i_{0}$

3) истинностные значения $\phi\left(\bar{d}_{1}, \bar{a}\right)$ и $\phi\left(\bar{d}_{2}, \bar{a}\right)$ в $M$ различны.

В этом случае $i_{0}$ называется неравной координатой рассматриваемой различающей пары. Если в этом случае $d_{1}^{i_{0}}<d_{2}^{i_{0}}$, то отрезок

$$
\left[d_{1}^{i_{0}}, d_{2}^{i_{0}}\right]=\left\{a \in I \mid d_{1}^{i_{0}} \leqslant a \leqslant d_{2}^{i_{0}}\right\}
$$

называется различающим для пары $\left(\bar{d}_{1}, \bar{d}_{2}\right)$ и обозначается $\operatorname{diff}\left[\bar{d}_{1}, \bar{d}_{2}\right]$.

Элемент $e$ множества $I$ называется определяющим для $L$-формулы $\phi(\bar{x}, \bar{y})$ и набора $\bar{a}$ элементов из $M$, если для любой окрестности $O$ в $I$ этого элемента $e$ существует такая различающая пара $\bar{d}_{1}, \bar{d}_{2}$ для $\phi(\bar{x}, \bar{y})$ и $\bar{a}$, что $\operatorname{diff}\left[\bar{d}_{1}, \bar{d}_{2}\right]$ содержится в $O$.

Ради краткости $L$-формулу $\phi(\bar{x}, \bar{y})$ будем называть $P$-сводимой в $(L, P)$ структуре $(M, I)$, если для нее существует сводящая бескванторная порядковая формула.

Совершенно очевидно, что $L$-формула $\phi(\bar{x}, \bar{y})$ является $P$-сводимой в $(L, P)$ структуре $(M, I)$, в которой $I$ является плотно полно упорядоченной неразличимой последовательностью, тогда и только тогда, когда существует такое натуральное число $k$, что для любого набора $\bar{a}$ из $M$ число определяющих элементов для $\phi$ и $\bar{a}$ не превосходит $k$.

Теорема 7.5. Если (L,P)-структура $(M, I)$ обладает первым свойством изолированности, то существуют $\left(N, J^{\prime}\right) \equiv(M, I)$ и такое бесконечное подмножество $J$ множества $J^{\prime}$, что $(N, J)$ является $P$-сводимой.

ДокАзАтельство. Рассмотрим $\left(2^{\omega}\right)^{+}$-насыщенную структуру $\left(N, J^{\prime}\right)$, элементарно эквивалентную структуре $(M, I)$. Понятно, что в $J^{\prime}$ есть такое подмножество $J$, что $(J,<)$ изоморфно упорядоченному обычным образом множеству действительных чисел.

Допустим, что структура $(N, J)$ не является $P$-сводимой. Пусть для $L$ формулы $\phi(\bar{x}, \bar{y}, z)$ не существует сводящей формулы, а для каждой $L$-формулы $\theta(\bar{v}, \bar{u})$, у которой длина набора $\bar{u}$ меньше длины набора $\bar{y}, z$, сводящая формула имеется.

Так как $L$-формула $\phi(\bar{x}, \bar{y}, z)$ не является $P$-сводимой, то для каждого целого положительного $i$ существует такой набор $\left(\bar{a}_{i}, b_{i}\right)$, что число определяющих элементов для $L$-формулы $\phi(\bar{x}, \bar{y}, z)$ и набора $\left(\bar{a}_{i}, b_{i}\right)$ больше $i$. Выберем попарно различные элементы $e_{i 1}, \ldots, e_{i i}$ из определяющих элементов для $L$-формулы $\phi(\bar{x}, \bar{y}, z)$ и набора $\left(\bar{a}_{i}, b_{i}\right)$. Для каждого из выбранных определяющих элементов $e_{i j}$ выбираем различающую пару $\left(\bar{d}_{1}^{i j}, \bar{d}_{2}^{i j}\right)$ таким образом, чтобы отрезки $\operatorname{diff}\left[\bar{d}_{1}^{i j}, \bar{d}_{2}^{i j}\right]$ не пересекались для разных $j$. Тройка $\left(\bar{d}_{1}^{i j}, \bar{d}_{2}^{i j}, \bar{d}_{3}^{i j}\right)$ получается таким образом, что набор $\bar{d}_{3}^{i j}$ получается из набора $\bar{d}_{2}^{i j}$ перемещением элемента, расположенного на месте неравной координаты этой различающей пары, вовнутрь отрезка $\operatorname{diff}\left[\bar{d}_{1}^{i j}, \bar{d}_{2}^{i j}\right]$. Понятно, что одна из пар $\left(\bar{d}_{1}^{i j}, \bar{d}_{3}^{i j}\right)$ и $\left(\bar{d}_{3}^{i j}, \bar{d}_{2}^{i j}\right)$ различающей не является. 
Понятно, что существует такое $\omega^{+}$-насыщенное элементарное расширение $\left(N^{\prime}, J^{\prime \prime \prime}, J^{\prime \prime}\right)$ структуры $\left(N, J^{\prime}, J\right)$, что в $N^{\prime}$ можно найти набор $(\bar{a}, b)$, для которого существует $\omega^{+}$таких определяющих элементов $e_{j}$, что множество $D$ всех элементов построенных описанным выше способом троек $\left(\bar{d}_{1}^{j}, \bar{d}_{2}^{j}, \bar{d}_{3}^{j}\right)$ является псевдоконечным в $N^{\prime}$ множеством мощности не меньше $\omega^{+}$. Ясно, что $D \subseteq J^{\prime \prime}$ и что структура $\left(N^{\prime}, J^{\prime \prime \prime}\right)$ элементарно эквивалентна структуре $(M, I)$. Пусть $E$ - множество всех элементов набора $\bar{a}$.

Рассмотрим тип $p=\operatorname{tp}(b /(E \cup D))$, и пусть $p$ изолируется подтипом $p_{0}=$ $\operatorname{tp}\left(b /\left(E \cup D_{0}\right)\right)$ для некоторого счетного подмножества $D_{0}$ множества $D$. Считаем $z$ единственной свободной переменной в формулах из $p$.

Понятно, что существует $\omega^{+}$пар $\left(\bar{d}_{1}^{j}, \bar{d}_{2}^{j}\right)$, ни один элемент которых не лежит в $D_{0}$ и для которых отрезки $\operatorname{diff}\left[\bar{d}_{1}^{j}, \bar{d}_{2}^{j}\right]$ не содержат элементов $D_{0}$. Такие пары назовем выделенными.

Пусть формула $\Phi(\bar{u}, \bar{v}, \bar{y}, z)$ говорит, что $(\bar{u}, \bar{v})$ является различающей парой для $L$-формулы $\phi(\bar{x}, \bar{y}, z)$ и набора $(\bar{y}, z)$. Очевидно, что $p_{0} \cup\left\{\Phi\left(\bar{d}_{1}, \bar{d}_{2}, \bar{a}, z\right)\right\}$ совместно для любых $\left(\bar{d}_{1}, \bar{d}_{2}\right)$. Покажем, что $p_{0} \cup\left\{\neg \Phi\left(\bar{d}_{1}, \bar{d}_{2}, \bar{a}, z\right)\right\}$ тоже совместно в $N^{\prime}$ для некоторой выделенной пары $\left(\bar{d}_{1}, \bar{d}_{2}\right)$. Если это так, то тип $p_{0}$ не изолирует тип $p$.

Допустим, что множество $p_{0} \cup\left\{\neg \Phi\left(\bar{d}_{1}, \bar{d}_{2}, \bar{a}, z\right)\right\}$ не является совместным в $N^{\prime}$ ни для какой выделенной пары $\left(\bar{d}_{1}, \bar{d}_{2}\right)$. Тогда для каждой выделенной пары $\left(\bar{d}_{1}, \bar{d}_{2}\right)$ существует такое свое конечное подмножество $p_{0}\left(\bar{d}_{1}, \bar{d}_{2}\right)$ типа $p_{0}$, для которого $p_{0}\left(\bar{d}_{1}, \bar{d}_{2}\right) \cup\left\{\neg \Phi\left(\bar{d}_{1}, \bar{d}_{2}, \bar{a}, z\right)\right\}$ не является совместным в $N^{\prime}$. Но понятно, что существует не менее $\omega^{+}$различных выделенных пар $\left(\bar{d}_{1}, \bar{d}_{2}\right)$, для которых $p_{0}^{\prime}=p_{0}\left(\bar{d}_{1}, \bar{d}_{2}\right)$ одинаково.

Так как множество формул $p_{0}^{\prime} \cup\left\{\neg \Phi\left(\bar{d}_{1}, \bar{d}_{2}, \bar{a}, z\right)\right\}$ конечно, можно рассмотреть конъюнкцию $\Psi\left(\bar{d}, \bar{d}_{1}, \bar{d}_{2}, \bar{a}, z\right)$ всех его формул, в которой $\bar{d}$ - это элементы из $D_{0}$. Формула $(\exists z) \Psi(\bar{w}, \bar{u}, \bar{v}, \bar{y}, z)$ является $P$-сводимой в структуре $\left(N^{\prime}, J^{\prime \prime \prime}\right)$. Пусть $\bar{c}$ - определяющие элементы для этой формулы и набора $\bar{a}$.

Пусть элементы наборов $\bar{d}_{1}, \bar{d}_{2}$ и $\bar{d}_{3}$ одинаково расположены относительно $\bar{c}$ и $\bar{d}$, пара $\left(\bar{d}_{1}, \bar{d}_{2}\right)$ является различающей для $(\bar{a}, b)$, а пара $\left(\bar{d}_{1}, \bar{d}_{3}\right)$ не является различающей для $(\bar{a}, b)$. Напомним, что набор $\bar{d}_{3}$ получается из набора $\bar{d}_{2}$ перемещением элемента, расположенного на месте неравной координаты различающей пары $\left(\bar{d}_{1}, \bar{d}_{2}\right)$, вовнутрь отрезка $\operatorname{diff}\left[\bar{d}_{1}, \bar{d}_{2}\right]$. Тогда истинность формулы $(\exists z) \Psi\left(\bar{d}, \bar{d}_{1}, \bar{d}_{2}, \bar{a}, z\right)$ равносильна истинности формулы $(\exists z) \Psi\left(\bar{d}, \bar{d}_{1}, \bar{d}_{3}, \bar{a}, z\right)$. Но формула $\Psi\left(\bar{d}, \bar{d}_{1}, \bar{d}_{3}, \bar{a}, z\right)$ истинна при $z=b$. Поэтому формула $(\exists z) \Psi\left(\bar{d}, \bar{d}_{1}, \bar{d}_{2}, \bar{a}, z\right)$ тоже истинна.

\section{8. Ограниченность сводимых теорий}

ОПРеДЕЛЕНиЕ 25. Такая $P$-сводимая $(L, P)$-структура $(N, J)$, что $N \equiv U$, $J$ является неразличимой последовательностью в $N$, ограничение $<$ на $J$ задает такой линейный порядок, что $(J,<)$ является упорядоченным обычным образом множеством действительных чисел, а $N$ является $\left(2^{\omega}\right)^{+}$-насыщенной системой, называется малой моделью для универсума $U$ и для элементарной теории этого универсума.

По теореме 7.1, каждая сводимая теория имеет малую модель. 
ОПРЕДЕЛЕНИЕ 26. Сводимая теория называется ограниченной, если каждая ее малая модель является $P$-ограниченной.

ТЕОРЕма 8.1. Каждая сводимая теория является ограниченной.

Из этой теоремы и теоремы 6.7 получаем

СлЕДСтвиЕ 8.2. Каждая сводимая теория обладает вторым свойством изолированности.

Из этого и теорем 6.3 и 6.4 получаем

СлЕДСТвИЕ 8.3. Для каждого сводимого универсума $U$ каждый расширеннъй локально генерический для конечных состояний над $U$ запрос эквивалентен для конечных состояний над $U$ некоторому ограниченному запросу.

Из этого и теоремы 7.2 получаем следующий результат.

СлЕДСТвИЕ 8.4. Для каждого универсума $U$ без независимой формуль каждый расширенный локально генерический для конечных состояний над $U$ запрос эквивалентен для конечных состояний надU некоторому ограниченному запросу.

Дальнейшая часть этого раздела посвящена доказательству теоремы 8.1. Теорема и доказательство придуманы С. М. Дудаковым (см. [8]).

В основе дальнейших построений лежит следующее замечание.

ЗАмечАниЕ 8.5 . Пусть $\psi(\bar{z}, \bar{y})$ - бескванторная порядковая формула, $a \leqslant b$ и наборы $\bar{c}, \bar{d}_{1}=d_{1,1}, \ldots, d_{1, m}, \bar{d}_{2}=d_{2,1}, \ldots, d_{2, m}$ удовлетворяют условиям:

1) $d_{1,1}<\cdots<d_{1, m}, d_{2,1}<\cdots<d_{2, m}$;

2) для любого $i \in\{1, \ldots, m\}$ и $j \in\{1,2\}$ если $d_{j, i}<a$ или $d_{j, i}>b$, то $d_{1, i}=d_{2, i}$

3) $\psi\left(\bar{c}, \bar{d}_{1}\right)$ истинно, а $\psi\left(\bar{c}, \bar{d}_{2}\right)$ ложно.

Тогда найдется такой элемент $e$ в наборе $\bar{c}$, что $a \leqslant e \leqslant b$.

Действительно, в противном случае наборы $\bar{d}_{1}$ и $\bar{d}_{2}$ реализуют один и тот же бескванторный порядковый тип над $\bar{c}$.

Пусть $(I,<)$ - плотный линейный порядок без концевых элементов. Напомним замечание 6.5 о том, что каждая порядковая формула $\psi(\bar{y})$ эквивалентна на $(I,<)$ бескванторной порядковой формуле.

Далее в этом разделе предполагаем, что $(M, I)$ является $P$-сводимой $(L, P)$ структурой и удовлетворяет условиям:

$I$ является неразличимой последовательностью в $M$, ограничение $<$ на $I$ задает такой линейный порядок, что $(I,<)$ является плотно полно упорядоченным множеством мощности $\lambda$ без концевых элементов, а $M$ является $\lambda^{+}$-насыщенной системой.

Лемма 8.6 [5]. Любая $P$-ограниченная L-формула эквивалентна в $(M, I)$ такой $Р$-ограниченной формуле, в которой сначала идут ограниченные кванторы существования, потом идут ограниченные кванторы всеобщности, а потом идет L-формула. 
Формулу, в которой сначала идут ограниченные кванторы существования, потом идут ограниченные кванторы всеобщности, а потом идет $L$-формула, будем называть ограниченной $\exists \forall$-формулой.

ДокАЗАТЕльство. Пусть $\psi_{\phi}(\bar{z}, \bar{w})$ является сводящей формулой для $\phi(\bar{y}, \bar{w})$. Другими словами, для каждой последовательности $\bar{m}$ элементов $M$ существует последовательность $\bar{c}_{\bar{m}} \in I$, для которой

$$
(\forall \bar{w} \in P)\left(\psi_{\phi}\left(\bar{c}_{\bar{m}}, \bar{w}\right) \leftrightarrow \phi(\bar{m}, \bar{w})\right) .
$$

Следовательно, формула

$$
(\forall \bar{y})(\exists \bar{z} \in P)(\forall \bar{w} \in P)\left(\psi_{\phi}(\bar{z}, \bar{w}) \leftrightarrow \phi(\bar{y}, \bar{w})\right)
$$

истинна в $(M, I)$.

Пусть $Q_{1}, \ldots, Q_{n}$ - некоторые знаки кванторов.

Тогда формула

$$
\left(Q_{1} w_{1} \in P\right) \ldots\left(Q_{n} w_{n} \in P\right) \phi(\bar{y}, \bar{w})
$$

эквивалентна формуле

$$
(\exists \bar{z} \in P)\left((\forall \bar{w} \in P)\left(\psi_{\phi}(\bar{z}, \bar{w}) \leftrightarrow \phi(\bar{y}, \bar{w})\right) \wedge\left(Q_{1} w_{1} \in P\right) \ldots\left(Q_{n} w_{n} \in P\right) \psi_{\phi}(\bar{z}, \bar{w})\right) .
$$

Формула

$$
\left(Q_{1} w_{1} \in P\right) \ldots\left(Q_{n} w_{n} \in P\right) \psi_{\phi}(\bar{z}, \bar{w})
$$

может быть заменена, по замечанию 6.5, на бескванторную. Лемма 8.6 доказана.

Для завершения доказательства теоремы 8.1 достаточно доказать, что формула, получаемая навешиванием квантора существования на ограниченную $\exists \forall$ формулу, эквивалентна $P$-ограниченной формуле в $(M, I)$.

Итак, рассмотрим $(L, P)$-формулу

$$
(\exists \bar{z} \in P)(\forall \bar{w} \in P) \phi(x, \bar{y}, \bar{z}, \bar{w}),
$$

в которой $\phi(x, \bar{y}, \bar{z}, \bar{w})$ является $L$-формулой. Ясно, что формула

$$
(\exists x)(\exists \bar{z} \in P)(\forall \bar{w} \in P) \phi(x, \bar{y}, \bar{z}, \bar{w})
$$

эквивалентна формуле

$$
(\exists \bar{z} \in P)(\exists x)(\forall \bar{w} \in P) \phi(x, \bar{y}, \bar{z}, \bar{w}),
$$

так как идущие подряд кванторы существования можно переставлять. По этой причине достаточно построить $P$-ограниченную формулу, эквивалентную в $(M, I)$ формуле

$$
(\exists x)(\forall \bar{w} \in P) \phi(x, \bar{y}, \bar{z}, \bar{w}) .
$$

Так как теперь можно объединить наборы $\bar{y}$ и $\bar{z}$, то достаточно построить $P$ ограниченную формулу, эквивалентную в $(M, I)$ формуле

$$
(\exists x)(\forall \bar{w} \in P) \phi(x, \bar{y}, \bar{w}),
$$

в которой $\phi(x, \bar{y}, \bar{w})$ является $L$-формулой.

В силу $\lambda^{+}$-насыщенности $M$, справедливо следующее замечание. 
ЗАмЕчАНИЕ 8.7. Истинность формулы

$$
(\exists x)(\forall \bar{w} \in P) \phi(x, \bar{y}, \bar{w})
$$

на заданном наборе $\bar{b}$ эквивалентна тому, что для каждого конечного $S \subseteq I$ можно найти такой $a$, что $\phi(a, \bar{b}, \bar{w})$ истинно в $M$ для всех наборов $\bar{w}$, элементы которых взяты из $S$.

Зафиксируем такую бескванторную порядковую формулу $\psi_{\phi}(\bar{z}, \bar{w})$, для которой формула

$$
(\forall x)(\forall \bar{y})(\exists \bar{z} \in P)(\forall \bar{w} \in P)\left(\psi_{\phi}(\bar{z}, \bar{w}) \leftrightarrow \phi(x, \bar{y}, \bar{w})\right)
$$

истинна в $(M, I)$. Пусть длина набора переменных $\bar{z}$ равна $L$, а длина набора $\bar{w}$ равна $N$.

Для набора $\bar{u}$ через $\operatorname{Set}(\bar{u})$ обозначается множество всех элементов набора $\bar{w}$. Мы будем дальше рассматривать $L$-формулы $\theta_{l_{1}, \ldots, l_{k}}(x, \bar{y}, \bar{u}, \bar{v})$, в которых

$$
\begin{gathered}
\bar{u}=u_{1,1}, \ldots, u_{1, l_{1}}, \ldots, u_{k, 1}, \ldots, u_{k, l_{k}}, \\
\bar{v}=v_{1}, \ldots, v_{k-1} \\
k, l_{1}, \ldots, l_{k} \geqslant 1
\end{gathered}
$$

и которые являются конъюнкциями $L$-формул, говорящих, что:

1) $u_{1,1}<\cdots<u_{1, l_{1}}<v_{1}<u_{2,1}<\cdots<u_{2, l_{2}}<v_{2}<\cdots<u_{k-1,1}<\cdots<$ $u_{k-1, l_{k-1}}<v_{k-1}<u_{k, 1}<\cdots<u_{k, l_{k}}$

2) $\phi(x, \bar{y}, \bar{w})$ для всех таких $\bar{w}$, что $\operatorname{Set}(\bar{w}) \subseteq \operatorname{Set}(\bar{u})$;

3) для каждого $v \in \operatorname{Set}(\bar{v})$

$$
\bigvee_{\substack{\operatorname{Set}(\bar{w}) \subseteq \\ \operatorname{Set}(\bar{u})}} \bigvee_{i=1}^{N} \neg \phi\left(x, \bar{y},\left\langle v \rightarrow_{i} \bar{w}\right\rangle\right)
$$

Здесь $\left\langle v \rightarrow_{i} \bar{w}\right\rangle$ обозначает набор, который получается из набора $\bar{w}$ заменой $w_{i}$ на $v$. Формула 3$)$ говорит о том, что для каждого $v \in \operatorname{Set}(\bar{v})$ найдется такой набор $\bar{w}$, составленный из элементов $\operatorname{Set}(\bar{u})$, что для некоторого $i \in\{1, \ldots, N\}$ выполняется $\neg \phi\left(x, \bar{y},\left\langle v \rightarrow_{i} \bar{w}\right\rangle\right)$.

ОПРЕДЕЛЕНИЕ 27. Формула $\theta_{l_{1}, \ldots, l_{k}}(x, \bar{y}, \bar{u}, \bar{v})$ называется обобщенной $(k, l)$ формулой, если те из чисел $l_{1}, \ldots, l_{k}$, которые отличны от 1, не меньше $l$, и хотя бы одно из чисел $l_{1}, \ldots, l_{k}$ больше 1 . Обобщенная $(k, l)$-формула называется $(k, l)$-формулой, если те из чисел $l_{1}, \ldots, l_{k}$, которые больше 1 , равны $l$.

$k$ называется числом серий в обобщенной $(k, l)$-формуле. Для $i \in\{1, \ldots, k\}$ набор $\bar{u}_{i}=u_{i, 1}, \ldots, u_{i, l_{i}}$ называется серией. Если $l_{i}=1$, эта серия называется точечной.

Пусть для элементов $\bar{a} \in I, \bar{b} \in I, x_{0}$ и $\bar{y}_{0}$ формула

$$
\theta_{l_{1}, \ldots, l_{k}}\left(x_{0}, \bar{y}_{0}, \bar{a}, \bar{b}\right)
$$

истинна в $M$, где

$$
\bar{a}=a_{1,1}, \ldots, a_{1, l_{1}}, \ldots, a_{k, 1}, \ldots, a_{k, l_{k}} .
$$


Тогда для $i \in\{1, \ldots, k\}$ набор

$$
\bar{a}_{i}=a_{i, 1}, \ldots, a_{i, l_{i}}
$$

тоже называется серией.

Пусть

$$
\begin{gathered}
{\left[\bar{a}_{i}\right]=\left\{d \in I \mid a_{i, 1} \leqslant d \leqslant a_{i, l_{i}}\right\},} \\
{[\bar{a}]_{\theta_{l_{1}, \ldots, l_{k}}}=\bigcup_{i=1}^{k}\left[\bar{a}_{i}\right] .}
\end{gathered}
$$

Строго говоря, разбиение набора $\bar{a}$ на серии и, значит, $[\bar{a}]_{\theta_{l_{1}, \ldots, l_{k}}}$ зависит от формулы $\theta_{l_{1}, \ldots, l_{k}}$. Но в дальнейшем мы будем опускать индекс $\theta_{l_{1}, \ldots, l_{k}}$ и писать просто $[\bar{a}]$, если будет понятно, о какой формуле идет речь.

Следующая лемма показывает, что в выполнимой обобщенной $(k, l)$-формуле число серий не может быть очень большим.

ЛЕмма 8.8. Если для элементов $\bar{a} \in I, \bar{b} \in I, x_{0}$ и $\bar{y}_{0}$ бормула

$$
\theta_{l_{1}, \ldots, l_{k}}\left(x_{0}, \bar{y}_{0}, \bar{a}, \bar{b}\right)
$$

истинна в $М$ и формула

$$
(\forall \bar{w} \in P)\left(\psi_{\phi}(\bar{c}, \bar{w}) \leftrightarrow \phi\left(x_{0}, \bar{y}_{0}, \bar{w}\right)\right)
$$

истинна в $(M, I)$, то в каждом отрезке $\left[b_{i}, a_{i+N, 1}\right]$ лежит хотя бы один элемент из набора $\bar{c}$. Следовательно, в выполнимой обобщенной $(k, l)$-формуле число серий не превосходит $N(L+1)$.

ДокАЗАТЕЛьство. Пусть $b_{i}-$ произвольный элемент набора $\bar{b}$. Выберем набор $\bar{d} \in \operatorname{Set}(\bar{a})$ такой, что

$$
(M, I) \models \neg \phi\left(x_{0}, \bar{y}_{0},\left\langle b_{i} \underset{j}{\rightarrow} \bar{d}\right\rangle\right)
$$

для некоторого $j$.

Будем менять элементы набора $\left\langle b_{i} \rightarrow_{j} \bar{d}\right\rangle$ таким образом, чтобы сохранилось старое упорядочение элементов набора между собой, чтобы элементы, расположенные вне отрезка $\left[b_{i}, a_{i+N, 1}\right]$, не изменились и чтобы измененный набор был составлен только из элементов множества $\operatorname{Set}(\bar{a})$. Если мы это сделаем, лемма будет следовать из замечания 8.5.

Элемент $b_{i}$ заменим на $a_{i+1,1}$. Если $a_{i+1,1}$ уже входит в $\bar{d}$, заменим его на следующий за ним элемент множества $\operatorname{Set}(\bar{a})$. Если и этот элемент входит в $\bar{d}$, заменим его на следующий за ним элемент множества $\operatorname{Set}(\bar{a})$ и так далее. Так как длина $\bar{d}$ равна $N$, то мы сделаем не более $N$ замен.

Пусть $Q$ есть $(N(L+1)+1)(N-1)+2$.

Обобщенная $(k, Q)$-формула называется обобщенной $k$-формулой. $(k, Q)$ формула называется $k$-формулой. Так как существует не более $2^{k}$ различных $k$-формул при заданном $k$, то для всех $k$ существует не более $2^{N(L+1)+1}-1$ различных выполнимых $k$-формул. 
Все эти формулы занумеруем натуральными числами

$$
0,1,2, \ldots, K
$$

следующим образом. Сначала нумеруем формулы с одной серией, затем с двумя сериями и так далее. Значит, формула с бо́льшим числом серий имеет больший номер. Формулы с одинаковым числом серий нумеруются таким образом, что сначала идут формулы с бо́льшим числом точечных серий, а формулы с одинаковым числом серий и одинаковым числом точечных серий нумеруются произвольным образом. Формулу с номером $i$ обозначим через $\gamma_{i}(x, \bar{y}, \bar{u}, \bar{v})$.

ЛЕмма 8.9 (о конечном множестве). Для любой обобщенной $k$-формулъ

$$
\theta_{l_{1}, \ldots, l_{k}}(x, \bar{y}, \bar{u}, \bar{v}),
$$

любых таких наборов $\bar{a} \in I, \bar{b} \in I, x_{0}$ и $\bar{y}_{0}$, для которых формула

$$
\theta_{l_{1}, \ldots, l_{k}}\left(x_{0}, \bar{y}_{0}, \bar{a}, \bar{b}\right)
$$

истинна в $M$, л любого такого конечного множества $G$, составленного из элементов множества I, что для любого такого $\bar{g}$, для которого $\operatorname{Set}(\bar{g}) \subseteq(G \cup \operatorname{Set}(\bar{a}))$, в $M$ истинно $\phi\left(x_{0}, \bar{y}_{0}, \bar{g}\right)$, существуют такие обобщенная $k^{\prime}$-формула

$$
\theta_{l_{1}^{\prime}, \ldots, l_{k^{\prime}}^{\prime}}\left(x, \bar{y}, \bar{u}^{\prime}, \bar{v}^{\prime}\right)
$$

$u \bar{a}^{\prime} \in I u \bar{b}^{\prime} \in I$, umo

$$
\theta_{l_{1}^{\prime}, \ldots, l_{k^{\prime}}}\left(x_{0}, \bar{y}_{0}, \bar{a}^{\prime}, \bar{b}^{\prime}\right)
$$

истинно в $M u(\operatorname{Set}(\bar{a}) \cup G) \subseteq \operatorname{Set}\left(\bar{a}^{\prime}\right)$. При этом $k^{\prime} \geqslant k$. Если $k^{\prime}=k$, то каждая серия только расширилась или не изменилась.

ДокАЗАТЕльство. Первым шагом включим каждый элемент $g$ множества $G$ в такую серию $\bar{a}$, чтобы между ней и $g$ не было никаких $b_{i}$.

После этого может оказаться, что длины некоторых неточечных серий меньше $Q$. Мы должны избавиться от таких серий. Будем делать это пошагово.

На каждом шаге рассматриваем произвольную "короткую” серию $\bar{a}_{i}$. Если в $\left[\bar{a}_{i}\right]$ существует элемент $h \in I$ такой, что для некоторых $\bar{d} \in \operatorname{Set}(\bar{a})$ имеет место $\neg \phi\left(x_{0}, \bar{y}_{0},\left\langle h \rightarrow{ }_{n} \bar{d}\right\rangle\right)$ для некоторого $n$, включаем это $h$ в новое $\bar{b}$, а серию $\bar{a}_{i}$ расщепляем на две более короткие. Таких шагов может быть не более $N(L+1)$. После того как такие шаги закончатся, каждый элемент из $\left[\bar{a}_{i}\right]$ можно добавить к серии $\bar{a}_{i}$.

ЛЕмма 8.10 (о внутренних элементах). Для любой обобщенной $k$-формуль

$$
\theta_{l_{1}, \ldots, l_{k}}(x, \bar{y}, \bar{u}, \bar{v}),
$$

любых таких наборов $\bar{a} \in I, \bar{b} \in I, x_{0} u \bar{y}_{0}$, для которых формула

$$
\theta_{l_{1}, \ldots, l_{k}}\left(x_{0}, \bar{y}_{0}, \bar{a}, \bar{b}\right)
$$

истинна в $M$, существуют такие $k^{\prime}$-формула

$$
\theta_{l_{1}^{\prime}, \ldots, l_{k^{\prime}}^{\prime}}\left(x, \bar{y}, \bar{u}^{\prime}, \bar{v}^{\prime}\right)
$$


$u \bar{a}^{\prime} \in I u \bar{b}^{\prime} \in I$, umo

$$
\theta_{l_{1}^{\prime}, \ldots, l_{k^{\prime}}^{\prime}}\left(x_{0}, \bar{y}_{0}, \bar{a}^{\prime}, \bar{b}^{\prime}\right)
$$

истинно в $M, \operatorname{Set}(\bar{a}) \subseteq\left[\bar{a}^{\prime}\right]$ и для любого такого набора $\bar{e}$, что $\operatorname{Set}(\bar{e}) \subseteq\left[\bar{a}^{\prime}\right]$, в $M$ выполняется $\phi\left(x_{0}, \bar{y}_{0}, \bar{e}\right)$. При этом $k^{\prime} \geqslant k$. Если $k^{\prime}=k$, то для любого такого набора $\bar{e}$, что $\operatorname{Set}(\bar{e}) \subseteq[\bar{a}]$, в $M$ выполняется $\phi\left(x_{0}, \bar{y}_{0}, \bar{e}\right)$.

ДокАЗАтЕльство. Сначала будем строить обобщенную $k^{\prime}$-формулу. Если для любого такого набора $\bar{e}$, что $\operatorname{Set}(\bar{e}) \subseteq[\bar{a}]$, в $M$ выполняется $\phi\left(x_{0}, \bar{y}_{0}, \bar{e}\right)$, то годятся

$$
\theta_{l_{1}, \ldots, l_{k}}(x, \bar{y}, \bar{u}, \bar{v})
$$

и $\bar{a}$ и $\bar{b}$.

Пусть $\operatorname{Set}(\bar{e}) \subseteq[\bar{a}]$ и $\neg \phi\left(x_{0}, \bar{y}_{0}, \bar{e}\right)$ истинно в $M$. Поочередно будем добавлять в $\bar{a}$ те элементы $e$ из $\bar{e}$, для которых формула

$$
\phi\left(x_{0}, \bar{y}_{0},\left\langle e \rightarrow_{n} \bar{d}\right\rangle\right)
$$

истинна для всех $n$ для любых таких $\bar{d}$, что $\operatorname{Set}(\bar{d}) \subseteq \operatorname{Set}(\bar{a})$. При этом некоторые серии будут расширены. Очевидно, что все элементы из $\bar{e}$ мы добавить не сможем. Следовательно, на некотором шаге для любого $e$ из оставшихся элементов $\bar{e}$ существует такое $\bar{d}$, что $\operatorname{Set}(\bar{d}) \subseteq \operatorname{Set}(\bar{a})$ и что в $M$ выполняется $\neg \phi\left(x_{0}, \bar{y}_{0},\left\langle e \rightarrow{ }_{n} \bar{d}\right\rangle\right)$ для некоторого $n$. Выберем такое $e$ и включим его в новое $\bar{b}$. При этом число серий увеличится. Если для новых наборов $\bar{a}$ и $\bar{b}$ и для любого такого набора $\bar{e}$, что $\operatorname{Set}(\bar{e}) \subseteq[\bar{a}]$, в $M$ выполняется $\phi\left(x_{0}, \bar{y}_{0}, \bar{e}\right)$, мы закончили построение. В противном случае выбираем такой набор $\bar{e}$, для которого $\operatorname{Set}(\bar{e}) \subseteq[\bar{a}]$ и $\neg \phi\left(x_{0}, \bar{y}_{0}, \bar{e}\right)$ истинно в $M$, и повторяем построение. Сделав не более $N(L+1)$ таких шагов, мы придем к случаю, когда для новых наборов $\bar{a}$ и $\bar{b}$ и для любого такого набора $\bar{e}$, что $\operatorname{Set}(\bar{e}) \subseteq[\bar{a}]$, в $M$ выполняется $\phi\left(x_{0}, \bar{y}_{0}, \bar{e}\right)$. При этом некоторые неточечные серии могут оказаться короткими, но, расширяя такую короткую серию $\left[\bar{a}_{i}\right]$ достаточным числом элементов $e \in\left[\bar{a}_{i}\right]$, мы сделаем число ее элементов не меньшим, чем $Q$.

Покажем теперь, как серии, содержащие более $Q$ элементов, сократить до серий с $Q$ элементами.

Для каждого $b_{i} \in \operatorname{Set}(\bar{b})$ в набор $\bar{d}$, для которого $\operatorname{Set}(\bar{d}) \subseteq \operatorname{Set}(\bar{a})$ и для которого в $M$ выполняется $\neg \phi\left(x_{0}, \bar{y}_{0},\left\langle b_{i} \rightarrow_{n} \bar{d}\right\rangle\right)$ для некоторого $n$, входит не более $N-1$ используемых элементов серии. Следовательно, нужно не более $(N(L+1)+1)(N-1)$ таких элементов серии. Нужны еще два крайних элемента серии. Всего нужно не более $(N(L+1)+1)(N-1)+2$ элемента. Остальные можно выбросить. Лемма 8.10 доказана.

Комбинируя последние две леммы, получаем следующий результат.

ЛЕмма 8.11. Для любой обобщенной $k$-формуль

$$
\theta_{l_{1}, \ldots, l_{k}}(x, \bar{y}, \bar{u}, \bar{v}),
$$

любых таких наборов $\bar{a} \in I, \bar{b} \in I, x_{0} u \bar{y}_{0}$, для которых формула

$$
\theta_{l_{1}, \ldots, l_{k}}\left(x_{0}, \bar{y}_{0}, \bar{a}, \bar{b}\right)
$$


истинна в $M$, июбого такого конечного множества $G$, составленного из элементов множества I, что для любого такого $\bar{g}$, для которого $\operatorname{Set}(\bar{g}) \subseteq$ $(G \cup \operatorname{Set}(\bar{a}))$, в $M$ истинно $\phi\left(x_{0}, \bar{y}_{0}, \bar{g}\right)$, существуют такая $k^{\prime}$-формула

$$
\theta_{l_{1}^{\prime}, \ldots, l_{k^{\prime}}^{\prime}}\left(x, \bar{y}, \bar{u}^{\prime}, \bar{v}^{\prime}\right)
$$

u maкue $\bar{a}^{\prime} \in I$ u $\bar{b}^{\prime} \in I$, иmo

$$
\theta_{l_{1}^{\prime}, \ldots, l_{k^{\prime}}^{\prime}}\left(x_{0}, \bar{y}_{0}, \bar{a}^{\prime}, \bar{b}^{\prime}\right)
$$

истинно в $M,(\operatorname{Set}(\bar{a}) \cup G) \subseteq\left[\bar{a}^{\prime}\right]$ и для любого такого набора $\bar{e}$, что $\operatorname{Set}(\bar{e}) \subseteq\left[\bar{a}^{\prime}\right]$, в $M$ выполняется $\phi\left(x_{0}, \bar{y}_{0}, \bar{e}\right)$. При этом $k^{\prime} \geqslant k$. Если $k^{\prime}=k$, то для любого такого набора $\bar{e}$, что $\operatorname{Set}(\bar{e}) \subseteq[\bar{a}]$, в $M$ выполняется $\phi\left(x_{0}, \bar{y}_{0}, \bar{e}\right)$.

Теперь мы готовы обратной индукцией по $i$ строить формулы

$$
\eta_{i}\left(x, \bar{y}, \bar{u}^{i}, \bar{v}^{i}\right)
$$

В качестве

$$
\eta_{K}\left(x, \bar{y}, \bar{u}^{K}, \bar{v}^{K}\right)
$$

берем $\gamma_{K}\left(x, \bar{y}, \bar{u}^{K}, \bar{v}^{K}\right)$. Так как $\gamma_{i}\left(x, \bar{y}, \bar{u}^{i}, \bar{v}^{i}\right)$ является $L$-формулой при любом $i$, то формула

$$
(\exists x) \eta_{K}\left(x, \bar{y}, \bar{u}^{K}, \bar{v}^{K}\right)
$$

является $L$-формулой и, значит, $P$-ограниченной формулой. Допустим, что формулы

$$
\eta_{j}\left(x, \bar{y}, \bar{u}^{j}, \bar{v}^{j}\right)
$$

построены для $j=K, \ldots, i+1$ так, что для $j=K, \ldots, i+1$ формула

$$
(\exists x) \eta_{j}\left(x, \bar{y}, \bar{u}^{j}, \bar{v}^{j}\right)
$$

эквивалентна $P$-ограниченной формуле. Это означает, что для каждого $j=$ $K, \ldots, i+1$ существует такая бескванторная порядковая формула

$$
\psi_{j}\left(\bar{f}_{j}, \bar{u}^{j}, \bar{v}^{j}\right)
$$

что

$$
(\forall \bar{y})\left(\exists \bar{f}_{j} \in P\right)\left(\forall \bar{u}^{j}, \bar{v}^{j} \in P\right)\left((\exists x) \eta_{j}\left(x, \bar{y}, \bar{u}^{j}, \bar{v}^{j}\right) \leftrightarrow \psi_{j}\left(\bar{f}_{j}, \bar{u}^{j}, \bar{v}^{j}\right)\right) .
$$

Длину набора $\bar{e}_{j}$ полагаем равной $\left(n_{j}+1\right)(N(L+1)+1)$, где $n_{j}$ является длиной набора $\bar{f}_{j}$. Пусть порядковая бескванторная формула

$$
\Theta_{j}\left(\bar{f}_{j}, \bar{e}_{j}\right)
$$

утверждает, что в каждом из открытых промежутков, на которые разбивает $I$ набор $\bar{f}_{j}$ (для удобства мы будем называть их $\bar{f}_{j}$-промежутками), содержится как минимум $N(L+1)+1$ различных элементов $\bar{e}_{j}$.

Пусть

$$
\eta_{i}\left(x, \bar{y}, \bar{u}^{i}, \bar{v}^{i}\right)
$$


есть следующая формула:

$$
\begin{aligned}
\left(\exists \bar{f}_{K}, \ldots, \bar{f}_{i+1} \in P\right) & \left(\bigwedge_{j=i+1}^{K}\left(\forall \bar{u}^{j}, \bar{v}^{j} \in P\right)\right. \\
\left.\left((\exists x) \eta_{j}\left(x, \bar{y}, \bar{u}^{j}, \bar{v}^{j}\right) \leftrightarrow \psi_{j}\left(\bar{f}_{j}, \bar{u}^{j}, \bar{v}^{j}\right)\right)\right) \wedge\left(\exists \bar{e}_{K}, \ldots, \bar{e}_{i+1} \in P\right) & \left.\left(\left(\bigwedge_{j=i+1}^{K} \Theta_{j}\left(\bar{f}_{j}, \bar{e}_{j}\right)\right) \wedge\left(\bigwedge_{\substack{\operatorname{Set}(\bar{g}) \subseteq \\
\left(G_{i} \cup \operatorname{Set}\left(\bar{u}^{i}\right)\right)}} \phi(x, \bar{y}, \bar{g})\right) \wedge \gamma_{i}\left(x, \bar{y}, \bar{u}^{i}, \bar{v}^{i}\right)\right)\right),
\end{aligned}
$$

где множество $G_{i}$ составлено из всех переменных наборов $\bar{f}_{K}, \bar{e}_{K}, \ldots, \bar{f}_{i+1}, \bar{e}_{i+1}$.

Ясно, что формула $(\exists x) \eta_{i}$ эквивалентна $P$-ограниченной формуле.

Пусть $\Phi_{i}(\bar{y})$ есть

$$
\begin{aligned}
\left(\exists \bar{f}_{i}\right. & \in P)\left(\left(\forall \bar{u}^{i}, \bar{v}^{i} \in P\right)\left((\exists x) \eta_{i}\left(x, \bar{y}, \bar{u}^{i}, \bar{v}^{i}\right) \leftrightarrow \psi_{i}\left(\bar{f}_{i}, \bar{u}^{i}, \bar{v}^{i}\right)\right)\right. \\
& \left.\wedge\left(\exists \bar{e}_{i} \in P\right)\left(\Theta_{i}\left(\bar{f}_{i}, \bar{e}_{i}\right) \wedge\left(\exists \bar{u}^{i}, \bar{v}^{i} \in P\right)(\exists x)\left(F_{i} \subseteq\left[\bar{u}^{i}\right]_{\gamma_{i}} \wedge \eta_{i}\left(x, \bar{y}, \bar{u}^{i}, \bar{v}^{i}\right)\right)\right)\right),
\end{aligned}
$$

где с помощью $F_{i}$ мы обозначили множество, составленное из всех элементов наборов $\bar{f}_{i}$ и $\bar{e}_{i}$. Сокращение $F_{i} \subseteq\left[\bar{u}^{i}\right]_{\gamma_{i}}$, очевидно, можно записать в виде порядковой бескванторной формулы.

Пусть, наконец, $\Phi(\bar{y})$ есть

$$
\bigvee_{j=0}^{K} \Phi_{j} .
$$

Очевидно, что все формулы $\Phi_{i}$ и, следовательно, формула $\Phi$ эквивалентны $P$-ограниченным формулам.

Лемма 8.12. Для заданного набора $\bar{y}_{0}$, для которого $\Phi\left(\bar{y}_{0}\right)$ истинно в $(M, I)$, пусть $\Phi_{i}\left(\bar{y}_{0}\right)$ истинно в $(M, I)$, а $\Phi_{j}\left(\bar{y}_{0}\right)$ ложно в $(M, I)$ для любого $j>i$. Пусть $\bar{f}_{i}, \bar{e}_{i}, x_{0}, \bar{a} \in I$ u $\bar{b} \in I$ выбраны так, что

$$
\begin{aligned}
&\left(\left(\forall \bar{u}^{i}, \bar{v}^{i} \in P\right)((\exists x)\right.\left.\eta_{i}\left(x, \bar{y}_{0}, \bar{u}^{i}, \bar{v}^{i}\right) \leftrightarrow \psi_{i}\left(\bar{f}_{i}, \bar{u}^{i}, \bar{v}^{i}\right)\right) \\
&\left.\wedge \Theta_{i}\left(\bar{f}_{i}, \bar{e}_{i}\right) \wedge F_{i} \subseteq[\bar{a}]_{\gamma_{i}} \wedge \eta_{i}\left(x_{0}, \bar{y}_{0}, \bar{a}, \bar{b}\right)\right)
\end{aligned}
$$

истинно в $(M, I)$.

Тогда для любых $\bar{a}^{\prime} \in I$ u $\bar{b}^{\prime} \in I$, расположенных относителъно $\bar{f}_{i}$ так же, как $\bar{a}$ и $\bar{b}$, существует такой $x_{0}^{\prime}$, что

$$
(M, I) \models \eta_{i}\left(x_{0}^{\prime}, \bar{y}_{0}, \bar{a}^{\prime}, \bar{b}^{\prime}\right)
$$

и для любого $\bar{g} \in\left[\bar{a}^{\prime}\right]_{\gamma_{i}}$ в $M$ истинно $\phi\left(x_{0}^{\prime}, \bar{y}_{0}, \bar{g}\right)$.

ДоказатеЛЬСтво. Так как

$$
\left(\forall \bar{u}^{i}, \bar{v}^{i} \in P\right)\left((\exists x) \eta_{i}\left(x, \bar{y}_{0}, \bar{u}^{i}, \bar{v}^{i}\right) \leftrightarrow \psi_{i}\left(\bar{f}_{i}, \bar{u}^{i}, \bar{v}^{i}\right)\right)
$$

истинно в $(M, I)$, то найдется такой $x_{0}^{\prime}$, для которого

$$
(M, I) \models \eta_{i}\left(x_{0}^{\prime}, \bar{y}_{0}, \bar{a}^{\prime}, \bar{b}^{\prime}\right) .
$$


В частности, для любого $\bar{g} \in\left(G_{i} \cup \operatorname{Set}\left(\bar{a}^{\prime}\right)\right)$ имеет место $\phi\left(x_{0}^{\prime}, \bar{y}_{0}, \bar{g}\right)$ в $M$.

Рассмотрим любой $\bar{g} \in\left[\bar{a}^{\prime}\right]_{\gamma_{i}}$. Предположим, что для него в $M$ истинно

$$
\neg \phi\left(x_{0}^{\prime}, \bar{y}_{0}, \bar{g}\right) .
$$

Тогда, согласно лемме 8.11, существуют такое $j>i$ и такие $\bar{a}^{\prime \prime}, \bar{b}^{\prime \prime}$, что

1) $\gamma_{j}\left(x_{0}^{\prime}, \bar{y}_{0}, \bar{a}^{\prime \prime}, \bar{b}^{\prime \prime}\right)$ истинно в $M$;

2) $\left(G_{i} \cup \operatorname{Set}\left(\bar{a}^{\prime}\right)\right) \subseteq\left[\bar{a}^{\prime \prime}\right]_{\gamma_{j}}$;

3) для всех таких $\bar{g}$, что $\operatorname{Set}(\bar{g}) \subseteq\left[\bar{a}^{\prime \prime}\right]_{\gamma_{j}}$, в $M$ истинно $\phi\left(x_{0}^{\prime}, \bar{y}_{0}, \bar{g}\right)$.

Рассмотрим произвольный $g \in\left(G_{j} \cup F_{j} \cup \operatorname{Set}\left(\bar{a}^{\prime \prime}\right)\right)$. Tак как $j>i$, то $\left(G_{j} \cup F_{j}\right) \subseteq G_{i}$. Следовательно, $g$ является элементом множества $G_{i} \cup \operatorname{Set}\left(\bar{a}^{\prime \prime}\right)$. Но тогда $g \in\left[\bar{a}^{\prime \prime}\right]_{\gamma_{j}}$. Из этого получаем, что для любого такого $\bar{g}$, что $\operatorname{Set}(\bar{g}) \subseteq\left(G_{j} \cup \operatorname{Set}\left(\bar{a}^{\prime \prime}\right)\right)$, в $M$ истинно $\phi\left(x_{0}^{\prime}, \bar{y}_{0}, \bar{g}\right)$. Из этого следует, что $\eta_{j}\left(x_{0}^{\prime}, \bar{y}_{0}, \bar{a}^{\prime \prime}, \bar{b}^{\prime \prime}\right)$ истинно в $(M, I)$. А из этого следует истинность $\Phi_{j}$ в $(M, I)$, что противоречит выбору $i$. Лемма 8.12 доказана.

Теперь мы приступаем к доказательству, что $\Phi$ как раз и является $P$-ограниченной формулой, которая эквивалентна

$$
(\exists x)(\forall \bar{w} \in P) \phi(x, \bar{y}, \bar{w})
$$

в $(M, I)$.

Так как $\gamma_{0}$ имеет только одну серию, то $\Phi_{0}(\bar{y})$ требует только существования для заданного конечного $S \subseteq I$ такого $x$, что $\phi(x, \bar{y}, \bar{w})$ истинно в $M$ для всех наборов $\bar{w}$, элементы которых взяты из $S$. Поэтому в $(M, I)$ из

$$
(\exists x)(\forall \bar{w} \in P) \phi(x, \bar{y}, \bar{w})
$$

следует $\Phi_{0}(\bar{y})$ и, значит, $\Phi(\bar{y})$.

Лемма 8.13. Из $\Phi(\bar{y})$ в $(M, I)$ следует

$$
(\exists x)(\forall \bar{w} \in P) \phi(x, \bar{y}, \bar{w}) .
$$

ДокАЗАТЕЛЬСтво. Для заданного набора $\bar{y}_{0}$, для которого $\Phi\left(\bar{y}_{0}\right)$ истинно в $(M, I)$, пусть $\Phi_{i}\left(\bar{y}_{0}\right)$ истинно в $(M, I)$, а $\Phi_{j}\left(\bar{y}_{0}\right)$ ложно в $(M, I)$ для любого $j>i$. Пусть $\bar{f}_{i}, \bar{e}_{i}, x_{0}, \bar{a} \in I$ и $\bar{b} \in I$ выбраны так, что утверждение (5) истинно в $(M, I)$.

По замечанию 8.7 , достаточно для заданного набора $\bar{y}_{0}$ и заданного конечного $S \subseteq I$ найти такой $x_{0}$, что $\phi\left(x_{0}, \bar{y}_{0}, \bar{w}\right)$ истинно в $M$ для всех наборов $\bar{w}$, элементы которых взяты из $S$.

Так как между соседними элементами $\bar{f}_{i}$ находится не меньше $N(L+1)+1$ разных элементов $\bar{e}_{i}$, а количество серий не превосходит $N(L+1)$, то хотя бы два из них покрываются одной и той же серией $\bar{a}$. Значит, в любом $\bar{f}_{i^{-}}$ промежутке есть элементы, которые покрываются одной и той же неточечной серией $\bar{a}$.

По лемме 8.12, для любых $\bar{a}^{\prime} \in I$ и $\bar{b}^{\prime} \in I$, расположенных относительно $\bar{f}_{i}$ так же, как $\bar{a}$ и $\bar{b}$, существует такой $x_{0}^{\prime}$, что

$$
(M, I) \models \eta_{i}\left(x_{0}^{\prime}, \bar{y}_{0}, \bar{a}^{\prime}, \bar{b}^{\prime}\right)
$$

и для любого $\bar{g} \in\left[\bar{a}^{\prime}\right]$ в $M$ истинно $\phi\left(x_{0}^{\prime}, \bar{y}_{0}, \bar{g}\right)$. 
По определению, $\operatorname{Set}\left(\bar{f}_{i}\right) \subseteq[\bar{a}]_{\gamma_{i}}$. Если $s \in S$ не входит в $[\bar{a}]$, то $s \notin \operatorname{Set}\left(\bar{f}_{i}\right)$ и в том же $\bar{f}_{i}$-промежутке, в который входит $s$, есть крайний элемент какой-то неточечной серии из $\bar{a}$. Так как $S$ конечно, можно найти такие наборы $\bar{a}^{\prime} \in I$ и $\bar{b}^{\prime} \in I$, расположенные относительно $\bar{f}_{i}$ так же, как и наборы $\bar{a}$ и $\bar{b}$, что $S \subseteq\left[\bar{a}^{\prime}\right]$.

\section{9. Активные запросы. Трансляция локально генерического запроса в активный для сводимых теорий}

В этом разделе рассматривается малая модель $(M, I)$ сводимой теории сигнатуры $L$. По теореме 8.1 , эта малая модель является $P$-ограниченной. Мы рассматриваем также базы данных со схемой $\rho$. Все рассматриваемые запросы предполагаются булевыми.

Пусть формула $\mathrm{AD}(x)$ задает активную область рассматриваемого состояния базы данных со схемой $\rho$. Ясно, что $\operatorname{AD}(x)$ можно считать $\rho$-формулой.

Напомним, что запрос называется активнъм, если он задается формулой, в которой все кванторы ограничены активной областью. Такие формулы тоже называются активными.

Более подробно, бескванторные формулы являются активными. Если $\Phi$ является активной формулой, то

$$
(\exists x)(\Phi \wedge \mathrm{AD}(x)) \quad \text { и } \quad(\forall x)(\mathrm{AD}(x) \rightarrow \Phi)
$$

тоже являются активными формулами. При помощи этих правил получается каждая активная формула.

Лемма 9.1. Каждый запрос, заданный Р-ограниченной $(<, \rho, P)$-формулой, эквивалентен в $(M, I)$ относительно конечных состояний над I некоторому активному запросу. Этот активный запрос эффективно строится по заданному запросу.

ДокАЗАТЕльство. Будем рассматривать два сорта переменных. Переменные первого сорта принимают значения из активной области, а переменные второго сорта принимают значения из той части $I$, которая лежит вне активной области. Ясно, что можно предполагать, что в формуле, задающей рассматриваемый запрос, кванторы берутся по переменным этих двух сортов.

Каждый бескванторный запрос является активным. Если на активную формулу навесить квантор по переменной первого сорта, она останется активной.

Итак, надо рассмотреть случай, когда на активную формулу навешивается квантор существования по переменной $у$ второго сорта.

Предварительно преобразуем саму активную формулу. Все атомные формулы, в которых участвует переменная второго сорта $y$, являются порядковыми. Так как активная область конечна, то либо у меньше наименьшего элемента активной области, либо больше наибольшего, либо в ней существуют такие крайние для $y$ элементы ${ }_{y} x$ и $x_{y}$, что ${ }_{y} x<x_{y}$, между ${ }_{y} x$ и $x_{y}$ нет элементов активной области и ${ }_{y} x<y<x_{y}$. Эти три случая рассматриваются аналогично. Рассмотрим только последний из них. Каждое неравенство $y<x$ для переменной первого сорта $x$ в рассматриваемой активной формуле можно заменить 
на $x_{y} \leqslant x$, и каждое неравенство $x<y$ можно заменить на $x \leqslant y x$, получив формулу $\psi$, а саму эту активную формулу заменить на

$$
\left(\exists x_{y}\right)\left(\exists_{y} x\right)\left({ }_{y} x<y<x_{y} \wedge\left(\forall x^{\prime}\right)\left(x^{\prime} \leqslant{ }_{y} x \vee x_{y} \leqslant x^{\prime}\right) \wedge \psi\right) .
$$

Здесь переменные $x_{y}, y x$ и $x^{\prime}$ имеют первый сорт. Теперь, перебирая всевозможные упорядочения используемых переменных второго сорта, для каждого такого упорядочения из этих переменных выберем наибольшую $z_{1}$ из меньших $y$ и наименьшую $z_{2}$ из больших $y$. Таким образом, надо найти такой $y$, что $z_{1}<y<z_{2}$ и ${ }_{y} x<y<x_{y}$. Из-за плотности порядка на $I$ для этого необходимо и достаточно, чтобы интервалы $\left(z_{1}, z_{2}\right)$ и $\left({ }_{y} x, x_{y}\right)$ пересекались. Это замечание позволяет переменную $y$ из формулы

$$
\left(\exists x_{y}\right)\left(\exists_{y} x\right)\left({ }_{y} x<y<x_{y} \wedge\left(\forall x^{\prime}\right)\left(x^{\prime} \leqslant{ }_{y} x \vee x_{y} \leqslant x^{\prime}\right) \wedge \psi\right)
$$

устранить.

Лемма 9.2. Каждый расширенный запрос эквивалентен в $(M, I)$ для конечных состояний над I запросу вида

$$
(\exists \bar{c} \in P)(\psi(\bar{c}) \wedge \theta(\bar{c})),
$$

в котором $\psi(\bar{c})$ является $(L, P)$-формулой, а $\theta(\bar{c})$ является Р-ограниченной $(<, \rho, P)$-формулой. Если $(M, I)$ является эффективно P-сводимой, то этот эквивалентный запрос эфбективно строится по заданному запросу.

ДоказАтельство. Будем рассматривать переменные двух сортов. Переменные первого сорта принимают значения из множества $I$, а значения переменных второго сорта не лежат в $I$. Можно считать, что каждый квантор берется по переменной некоторого сорта. Переменные второго сорта не принимают значений из активной области и не участвуют в атомных формулах вида $R(\bar{x})$ для $R \in \rho$. Подформулы вида $R(\bar{x}, \bar{y})$ для $R \in L$, где переменные $\bar{x}$ берутся из $I$, а $\bar{y}$ не из $I$, надо заменить на $\psi_{R}\left(\bar{x}, \bar{z}_{\bar{y}}\right)$, взяв конъюнкцию полученной формулы и всех формул

$$
(\forall \bar{x} \in P)\left(\psi_{R}\left(\bar{x}, \bar{z}_{\bar{y}}\right) \leftrightarrow R(\bar{x}, \bar{y})\right)
$$

и навесив на эту конъюнкцию все блоки кванторов $\left(\exists \bar{z}_{\bar{y}} \in P\right)$. Каждая бескванторная формула после этого примет вид

$$
(\exists \bar{c} \in P)(\psi(\bar{c}, \bar{x}, \bar{y}) \wedge \theta(\bar{c}, \bar{x}, \bar{y})),
$$

где $\psi(\bar{c}, \bar{x}, \bar{y})-(L, P)$-формула и $\theta(\bar{c}, \bar{x}, \bar{y})-P$-ограниченная $(<, \rho, P)$-формула. Отрицание формулы такого вида имеет вид

$$
(\forall \bar{c} \in P)(\neg \psi(\bar{c}, \bar{x}, \bar{y}) \vee \neg \theta(\bar{c}, \bar{x}, \bar{y})) .
$$

Заменив $\neg \psi(\bar{c}, \bar{x}, \bar{y})$ на $\xi(\bar{c}, \bar{x}, \bar{d})$, взяв конъюнкцию полученной формулы и формулы

$$
(\forall \bar{w} \in P)(\xi(\bar{w}, \bar{d}) \leftrightarrow \neg \psi(\bar{w}, \bar{y}))
$$


и навесив на эту конъюнкцию блок кванторов $(\exists \bar{d} \in P)$, получим формулу нужного вида, эквивалентную отрицанию рассматриваемой формулы. Навешивание на эту формулу квантора существования по переменной первого сорта сохраняет ее вид, а навешивание квантора существования по переменной второго сорта сводится к навешиванию его на $\psi(\bar{c}, \bar{x}, \bar{y})$.

ЛЕмма 9.3. Каждый расширенный запрос эквивалентен в $(M, I)$ для конечных состояний над I запросу $\eta$, задаваемому $P$-ограниченной $(<, \rho, P)$-формулой. Если I является эфбективно неразличимой в $(M, I)$ и $(M, I)$ является эффективно Р-сводимой, то $\eta$ эффективно строится по заданному запросу.

ДокАЗАТЕЛЬство. Так как, по теореме 6.7, I является неразличимой последовательностью в $(M, I)$, то $\psi(\bar{c})$ эквивалентна некоторой бескванторной порядковой формуле $\gamma(\bar{c})$.

СлЕДСТвИЕ 9.4. Каждый расширенный запрос в $(M, I)$ эквивалентен для конечных состояний над I активному запросу $\theta$, в котором $\theta$ является $(<, \rho)$ формулой. Если I является эфбективно неразличимой в $(M, I)$ u $(M, I)$ является эффективно Р-сводимой, то $\theta$ эффективно строится по заданному запросу.

\section{ДоКАЗАТЕЛЬСтво. Надо использовать лемму 9.1.}

ТЕОрема 9.5. Если I является эффективно неразличимой в $(M, I)$ и $(M, I)$ является эффективно Р-сводимой, то каждый локально генерический расширенный запрос эквивалентен в $(M, I)$ для конечных состояний активному ограниченному запросу и этот активный запрос эффективно строится по заданному расширенному запросу.

Лемма 9.6. Если для универсума U сигнатуры L без независимой формуль существует алгоритм, который по каждой замкнутой $L$-формуле определяет, истинна ли эта формула в $U$, то малая модель $(M, I)$ для $\operatorname{Th}(U)$ является эбфективно Р-сводимой.

ДокАЗАТЕЛьство. Как замечено в начале доказательства теоремы 7.2, достаточно по $L$-формуле $\phi(\bar{x}, \bar{y})$ эффективно найти такое $n$, для которого любые наборы значений

$$
\bar{a}_{1}, \ldots, \bar{a}_{n}
$$

для набора переменных $\bar{x}$ из носителя структуры $M$ и, тем более, из $I$ не удовлетворяют условию:

(А) для любого $\eta \subseteq\{1, \ldots, n\}$ найдется такой набор значений $\bar{b}_{\eta}$ для набора переменных $\bar{y}$, что

$$
\eta=\left\{i \in\{1, \ldots, n\} \mid M \models \phi\left(\bar{a}_{i}, \bar{b}_{\eta}\right)\right\} .
$$

Так как $U$ и $M$ элементарно эквивалентны, достаточно эффективно найти такое $n$, для которого любые наборы значений

$$
\bar{a}_{1}, \ldots, \bar{a}_{n}
$$

для набора переменных $\bar{x}$ из носителя структуры $U$ не удовлетворяют условию (А). 
Так как это условие для заданного $n$ задается $L$-формулой

$$
\gamma_{n}\left(\bar{a}_{1}, \ldots, \bar{a}_{n}\right)
$$

то надо найти такое $n$, для которого истинна формула

$$
\left(\forall \bar{a}_{1}, \ldots, \bar{a}_{n}\right) \neg \gamma_{n}\left(\bar{a}_{1}, \ldots, \bar{a}_{n}\right) .
$$

Перебираем $n$, начиная с $n=1$, до тех пор, пока не найдем нужное.

Таким образом, остается научиться строить эффективно неразличимую последовательность. По теореме компактности Мальцева (теорема 3.5), достаточно уметь строить эффективно почти неразличимую счетную последовательность, в которой условие неразличимости выполняется для каждой формулы, начиная с некоторого момента. Такую последовательность легко строить для арифметики Пресбургера и для поля действительных чисел. По этой причине для этих теорий каждый расширенный запрос эффективно перерабатывается в эквивалентный активный ограниченный запрос.

\section{0. Теория с независимой формулой}

Через $\mathbb{N}$ обозначаем множество натуральных чисел. В [16] доказано, что элементарная теория структуры $\left(\mathbb{N},<,+,\left.\right|_{p}\right)$, где $p>1$ - натуральное число и $\left.x\right|_{p} y$ определено как

$$
(\exists u)(\exists k)\left(x=p^{u} \wedge y=k x\right),
$$

разрешима.

Тривиально и замечено в $[21]$, что $\left(\mathbb{N},<,+,\left.\right|_{p}\right)$ имеет независимую формулу.

Действительно, пусть $x_{i}=p^{u_{i}}$ для $i=1, \ldots, m$, где $u_{1}, \ldots, u_{m}$ попарно различны. Пусть $y=x_{1}+\cdots+x_{m}$. Тогда формула

$$
(\exists u)(\exists v)\left(\left.p x\right|_{p} u \wedge v<x \wedge y=x+u+v\right)
$$

выполняется для $x$ тогда и только тогда, когда $x \in\left\{x_{1}, \ldots, x_{m}\right\}$. Поэтому (6) является независимой формулой.

Пусть $f_{p}(x)=y$ означает, что $\left(\left.y\right|_{p} x \wedge \neg\left(\left.p y\right|_{p} x\right)\right)$.

Тогда $\left(\left.y\right|_{p} x\right)$ эквивалентно

$$
\left(f_{p}(x) \geqslant y \wedge f_{p}(y)=y\right)
$$

Поэтому структуры $\left(\mathbb{N},<,+,\left.\right|_{p}\right)$ и $\left(\mathbb{N},<,+, f_{p}\right)$ интерпретируемы друг в друге.

Заметим еще, что структуры $\left(\mathbb{N},<,+,\left.\right|_{2}\right)$ и $(\mathbb{N},<, E)$ тоже интерпретируемы друг в друге, где $x \in y$ означает, что

$$
(\exists z)(\exists u)\left(y=z+x+u \wedge z<\left.x \wedge 2 x\right|_{2} u\right) .
$$

В самом деле, $\left(\left.x\right|_{2} y\right)$ выполняется тогда и только тогда, когда

$$
(x \in x \wedge(\forall v)(v<x \rightarrow v \not \equiv y)) .
$$


Кроме того, $x+y=z$ выполняется тогда и только тогда, когда

$$
\begin{aligned}
& (\exists u)(1 \not \subset u \wedge(\forall v)(\forall w)((v \in v \wedge w E w \\
& \wedge v<w \wedge(\forall t)((t \in t \wedge v<t) \rightarrow w \leqslant t)) \\
& \rightarrow((w E u \leftrightarrow((v E x \wedge v E y) \vee(v E x \wedge v \in u) \vee(v \in y \wedge v E u))) \\
& \wedge(v \in z \leftrightarrow((v \in x \wedge v \in y \wedge v \in u) \\
& \vee(v \in x \wedge v \not \equiv u \wedge v \not \equiv y) \vee(v \in y \wedge v \not \equiv u \wedge v \not \equiv x)))))) \text {. }
\end{aligned}
$$

Заметим, наконец, что $x<y$ можно использовать только для таких $x$ и $y$, для которых $x \in x$ и $y \in y$. Действительно, $u<v$ для произвольных $u$ и $v$ выражается как

$$
(\exists x)(x \in v \wedge x \not \equiv u \wedge(\forall y)(x<y \rightarrow(y \in u \leftrightarrow y \boxminus v)))
$$

Итак, мы рассматриваем структуру $(\mathbb{N},<, \mathbb{E})$, где $<$ определено только на множестве $\{x \mid x \in x\}$. Эта структура представляет собой двухосновную структуру, в которой первый носитель - натуральные числа с операцией следования, второй - конечные подмножества натуральных чисел и имеется еще обычное отношение включения натуральных чисел в подмножество. Поэтому элементарная теория этой двухосновной структуры совпадает со слабой монадической теорией второго порядка одного следования. Так что разрешимость этой элементарной теории и, следовательно, разрешимость элементарной теории структуры $\left(\mathbb{N},<,+,\left.\right|_{p}\right)$ следуют из знаменитой теоремы Рабина о разрешимости слабой монадической теорией второго порядка двух следований (см. [22], [23]). Эту структуру можно также рассматривать как идеал Фреше атомной булевой алгебры, в которой множество атомов упорядочено по типу натуральных чисел. Роль атомов в этом случае выполняют такие $a$, для которых $a \in a$. В дальнейшем, эти элементы мы и будем называть атомами.

Теперь мы собираемся доказать, что трансляционная теорема не выполняется для структуры $(\mathbb{N},<, E)$.

Мы рассматриваем схему баз данных

$$
\rho=\left\langle S,<_{c}, F, R, 0_{c}, c\right\rangle,
$$

в которой $S$ является трехместным символом отношения, $F$ и $<_{c}-$ двухместные символы отношений, $R$ - одноместный символ отношения, а $c$ и $0_{c}-$ символы выделенных элементов.

Пусть $\rho$-формула $\mathrm{AD}_{\rho}(x)$ такова, что для любого универсума $U$, для любого $a \in U$ и для любого $\rho$-состояния $s$ в $U \operatorname{AD}_{\rho}(a)$ истинно тогда и только тогда, когда $а$ лежит в активной области состояния $s$.

Скажем, что все кванторы в формуле $\Psi$ ограничены формулой $\mathrm{AD}_{\rho}(x)$, если для любой подформулы $(\forall y) \Phi$ или $(\exists y) \Phi$ формулы $\Psi$, в которой $y$ является переменной, эта переменная $y$ отлична от всех связанных переменных формулы $\operatorname{AD}_{\rho}(x),(\forall y) \Phi$ при этом понимается как $(\forall y)\left(\operatorname{AD}_{\rho}(y) \rightarrow \Phi\right)$ и $(\exists y) \Phi$ понимается как $(\exists y)\left(\operatorname{AD}_{\rho}(y) \wedge \Phi\right)$.

Пусть $\phi$ является конъюнкцией следующих $\rho$-высказываний, в которых все кванторы ограничены формулой $\operatorname{AD}_{\rho}(x)$ : 
а) высказывание, говорящее, что $<_{c}$ является линейным порядком на активной области с максимальным элементом $c$ и минимальным элементом $0_{c}$;

b) высказывание, говорящее, что $S$ - бинарная операция;

c) $(\forall x)(\forall y)(\forall u)(\forall v)(\exists w)(S(x, 0, x) \wedge((S(x, y, u) \wedge \theta(y, v)) \rightarrow$ $(S(x, v, w) \wedge \theta(u, w))))$, где $\theta(a, b)$ есть сокращение для

$$
\left((a=c \wedge b=c) \vee(\forall x)\left(a<_{c} b \wedge\left(a<_{c} x \rightarrow\left(b<_{c} x \vee x=b\right)\right)\right)\right) ;
$$

таким образом, $\theta(a, b)$ говорит, что $b$ является следующим после $a$ элементом в упорядочении $<_{c}$, если $a$ отлично от $c$, и $b$ есть $c$, если $a$ есть $c$; вся формула говорит, что $S$ определяет сложение $+_{c}$ на начальном отрезке натуральных чисел, представленном активной областью;

d) высказывание, говорящее, что $F$ определяет унарную операцию,

$$
(\exists v)(F(0, v) \wedge \theta(0, v))
$$

и

$$
\begin{aligned}
(\forall x)(\forall y)(\forall z)((F(x, y) & \wedge \theta(x, z)) \rightarrow(\exists u)\left(\exists w_{1}\right)\left(\exists w_{2}\right) \\
(F(z, u) & \left.\left.\wedge S\left(y, y, w_{1}\right) \wedge S\left(w_{1}, y, w_{2}\right) \wedge S\left(w_{2}, y, u\right)\right)\right) .
\end{aligned}
$$

Эта формула говорит, что операция, определяемая $F$, есть $4^{x}$;

е) формула, которая говорит, что для любых двух идущих один непосредственно за другим элементов $x$ и $y$ из $R$ выполняется $F(x, y)$ :

$$
\begin{aligned}
(\forall x)(\forall y)\left(\left(R(x) \wedge R(y) \wedge x<_{c} y \wedge(\forall z)\left(\left(x<_{c} z \wedge R(z)\right)\right.\right.\right. & \\
& \left.\left.\left.\rightarrow\left(z=y \vee y<_{c} z\right)\right)\right) \rightarrow F(x, y)\right)
\end{aligned}
$$

f) $R\left(0_{c}\right)$ и $R(c)$;

g) высказывание, говорящее для любого такого $x$ из $R$, что $x<_{c} c$, любого такого $y$, что $y<_{c} x$, и любого такого $z$, что $z<_{c} 4^{y}$, о справедливости неравенства

$$
4^{y}+{ }_{c} 4^{y}+{ }_{c} 4^{y}+{ }_{c} z<_{c} c ;
$$

через $4^{a}$ обозначен элемент системы, для которого выполняется $F\left(a, 4^{a}\right)$; через $\left(a+{ }_{c} b\right)$ обозначен элемент системы, для которого выполняется $S\left(a, b,\left(a+{ }_{c} b\right)\right)$.

Далее мы рассматриваем только такие $\rho$-состояния $s$ для $(\mathbb{N},<, \mathbb{E})$, которые удовлетворяют $\phi$. Кроме того, мы предполагаем, что $<_{c}$ есть ограничение $<$ на активную область состояния $s$.

Теперь мы собираемся предложить расширенное высказывание, говорящее для любого $\rho$-состояния $s$, что $s(R)$ четно.

Любой элемент из $(\mathbb{N},<, E)$ можно рассматривать как конечное множество атомов. Для любого элемента $a$ из активной области состояния $s$, отличного от $s(c)$, существует следующий относительно < элемент $b$ активной области и можно образовать множество $((a \backslash b) \cup(b \backslash a))$. Возьмем максимальный элемент в этом множестве $((a \backslash b) \cup(b \backslash a))$. Этот максимальный атом $f_{s}(a)$ определяется 
однозначно. В качестве $\gamma(x, y)$ возьмем расширенную формулу, говорящую для $x$ из активной области, отличного от максимального элемента активной области, что $y$ является максимальным элементом в симметрической разности $x$ и следующего за ним элемента активной области. Это определяет функцию $f_{s}$ из активной области состояния $s$ в натуральные числа.

ЛЕмма 10.1. Пусть $1_{c}$ - элемент $\mathrm{AD}(s)$, следующий по порядку за $0_{c}$. Для любого а $\in s(R)$, отличного от $s(c), s\left(0_{c}\right)$ u $s\left(1_{c}\right)$, существует такой $b \in$ $\mathrm{AD}(s)$, что $a<b \leqslant 4^{a} u f_{s}(b)$ отлично от любого $f_{s}(d)$ для любого такого $d \in \operatorname{AD}(s)$, чmo $d \leqslant a$.

ДокАЗАТЕЛЬСтво. Пусть $a \in s(R)$ и $s\left(1_{c}\right)<a<s(c)$. Пусть

$$
X=\left\{f_{s}(d) \mid d \in \operatorname{AD}(s), d \leqslant a\right\} .
$$

Предположим, что $f_{s}(b) \in X$ для любого такого $b \in \mathrm{AD}(s)$, что $a<b \leqslant 4^{a}$.

Для каждого $b \in \mathrm{AD}(s)$ рассмотрим такое подмножество $h(b)$ множества $X$, что для $e \in X$ тогда и только тогда $e \in h(b)$, когда $e \in b$.

Если $a<b_{1}<b_{2} \leqslant 2^{a}, b_{1} \in \mathrm{AD}(s), b_{2} \in \mathrm{AD}(s)$, то $h\left(b_{1}\right)<h\left(b_{2}\right)$ в $(\mathbb{N},<, E)$.

Действительно, если $b_{2}$ является следующим после $b_{1}$ относительно < элементом активной области состояния $s$, то максимальное число в $\left(\left(b_{1} \backslash b_{2}\right) \cup\right.$ $\left.\left(b_{2} \backslash b_{1}\right)\right)$ лежит в $b_{2}$. По нашему построению, это максимальное число лежит в $X$. Следовательно, в этом случае $h\left(b_{1}\right)<h\left(b_{2}\right)$ в $(\mathbb{N},<, \mathbb{E})$. Из транзитивности отношения $<$ следует, что $h\left(b_{1}\right)<h\left(b_{2}\right)$ в случае, когда $a<b_{1}<b_{2} \leqslant 2^{a}$, $b_{1} \in \mathrm{AD}(s)$ и $b_{2} \in \mathrm{AD}(s)$.

Допустим, что имеется ровно $n$ элементов из $\mathrm{AD}(s)$, которые меньше $a$. Тогда число всех элементов множества $X$ не превосходит $n+1$. Значит, число всех подмножеств множества $X$ не превосходит $2^{n+1}$. Однако число всех элементов множества $\mathrm{AD}(s)$, расположенных между $a$ и $4^{a}$ и отличных от $a$, равно $4^{n}-n$, что больше $2^{n+1}$ при $n \geqslant 2$. Получили противоречие.

Легко понять, что, используя формулу $\gamma$, можно построить формулу $\beta(x, y)$, которая истинна для $(a, b)$, если $b$ является минимальным элементом активной области, удовлетворяющим условию леммы.

TEOPEMA 10.2. Запрос

"Состояние s удовлетворяет ф и число элементов множества $s(R)$ четно"

является локально генерическим и задается расширенным высказыванием.

ДокАЗАТЕЛЬСтво. Для $a \in s(R)$ и $s\left(1_{c}\right) a<s(c)$ расширенная формула $\beta(a, b)$ говорит, что $b \in \mathrm{AD}(s)$ является минимальным среди всех таких элементов $d$, что $d \in \operatorname{AD}(s), a<d \leqslant 4^{a}$ и атом $f_{s}(d)$ отличен от любого атома $f_{s}(e)$ для любого $e \in \mathrm{AD}(s), e \leqslant a$. Используя $\beta$, можно построить формулу

$$
\alpha(x, z)=(\exists y)(\beta(x, y) \wedge \gamma(y, z)) .
$$

Формула $\alpha(x, z)$ для каждого $x \notin\left\{0_{c}, 1_{c}, c\right\}$ выбирает уникальный атом $z$. Осталось написать формулу, говорящую, что множество $A$ всех выбранных таким образом атомов $z$ нечетно. 
Мы предлагаем следущую формулу. Эта формула говорит, что существует множество атомов $Y$, содержащее первый элемент множества $A$, содержащее следующий элемент множества $A$ тогда и только тогда, когда $Y$ не содержит рассматриваемый элемент множества $A$, и содержащее последний элемент множества $A$.

Осталось доказать следующую теорему.

ТЕОРема 10.3. Не существует ограниченного высказывания, задающего запрос из теоремы 10.2 .

Из этой теоремы и теоремы 10.2 вытекают следующие результаты.

СлЕДСтвиЕ 10.4. Трансляиионная теорема не выполняется для теории системы $(\mathbb{N},<, \in)$.

СлЕДСтвиЕ 10.5. Существует обогащение арифметики Пресбургера, которое имеет разрешимую элементарную теорию и для которого трансляиионная теорема не выполняется.

Для доказательства теоремы 10.3 надо описать $(<, \rho)$-теории удовлетворяющих $\phi$ конечных $\rho$-состояний структуры $(\mathbb{N},<)$. Напомним, что $\phi$ является конъюнкцией формул а)-g). Оказывается, что для каждого $(<, \rho)$-высказывания $\psi$ можно построить бескванторную порядковую формулу, говорящую, что $c$ (как натуральное число) удовлетворяет некоторым неравенствам с заданными натуральными числами, и эквивалентную $\psi$ для удовлетворяющих $\phi$ конечных $\rho$-состояний структуры $(\mathbb{N},<)$. Подробности построения такой бескванторной порядковой формулы рутинны, но достаточно громоздки, и здесь мы их не приводим. Понятно, что такая бескванторная порядковая формула не может утверждать четность множества $s(R)$.

\section{Список литературы}

[1] E. F. Codd, "A relational model for large shared data banks", Commun. ACM, 13:6 (1970), 377-387.

[2] E. F. Codd, "Relational completeness of data base sublanguages", Database systems, ed. R. Rustin, Prentice-Hall, Englewood Cliffs, NJ, 1972, 33-64.

[3] O. V. Belegradek, A.P. Stolboushkin, M. A. Taitslin, "Extended order-generic queries", Ann. Pure Appl. Logic, 97:1-3 (1999), 85-125.

[4] M. Benedikt, G. Dong, L. Libkin, L. Wong, "Relational expressive power of constraint query languages", J. ACM, 45:1 (1998), 1-34.

[5] J. Baldwin, M. Benedikt, "Stability theory, permutations of indiscernibles, and embedded finite models", Trans. Amer. Math. Soc., 352:11 (2000), 4937-4969.

[6] С. М. Дудаков, "Трансляционная теорема в теориях предикатных обогащений начального фрагмента нестандартных моделей арифметики Пресбургера", Сложные системы: обработка информации, моделирование и оптимизация, Твер. гос. ун-т, Тверь, 2002, 24-37.

[7] С. М. Дудаков, “Трансляционный результат для расширений арифметики Пресбургера одноместной функцией, согласованной со сложением", Матем. заметки, 76:3 (2004), 362-371.

[8] С. М. Дудаков, "Трансляционная теорема для теорий $I$-сводимых алгебраических систем", Изв. РАН. Сер. матем., 68:5 (2004), 67-90. 
[9] И.В. Попов, "Элиминация кванторов в некоторых обогащениях арифметики Пресбургера", Сложные системы: обработка информаиии, моделирование и оптимизачия, Твер. гос. ун-т, Тверь, 2002, 38-47.

[10] М. А. Тайцлин, “Трансляционные результаты в теории баз данных”, Сложные системы: обработка информации, моделирование и оптимизация, Твер. гос. ун-т, Тверь, 2002, 5-23.

[11] М.А. Тайцлин, "Ограниченные псевдоконечная однородность и изолированность”, Вестник Твер. гос. ун-та, сер. Прикладная математика, вып. 2, № 2(2), Твер. гос. ун-т, Тверь, 2003, 5-15.

[12] О.В. Белеградек, А. П. Столбоушкин, М. А. Тайцлин, “Базы данных над фиксированным бесконечным универсумом", Программирование, 1998, № 1, 6-17.

[13] С.М. Дудаков, "Разрешимая теория без трансляционной теоремы”, Вестник Твер. гос. ун-та, сер. Прикладная математика, вып. 2, №6(12), Твер. гос. ун-т, Тверь, 2005, 23-26.

[14] С. М. Дудаков, "Выразительная сила языков запросов первого порядка для баз данных на неупорядоченном случайном графе”, Вестник НовгГУ, 2005, № 5, 4550.

[15] M. A. Taitslin, "A general condition for collapse results", Ann. Pure Appl. Logic, 113:1-3 (2002), 323-330.

[16] A. Blumensath, E. Grädel, "Automatic structures", Proceedings of the 15th Annual IEEE Symposium on Logic in Computer Science, Santa Barbara, CA, 2000, 51-62.

[17] Т. Йех, Теория множеств и метод форсинга, Мир, М., 1973.

[18] Г. Кейслер, Ч. Ч. Чэн, Теория моделей, Мир, М., 1977.

[19] S. Shelah, Classification theory and the number of nonisomorphic models, Stud. Logic Found. Math., 92, North-Holland, Amsterdam, 1990.

[20] S. Shelah, "Stability, the f.c.p., and superstability; model theoretic properties of formulas in first order theory", Ann. Math. Logic, 3:3 (1971), 271-362.

[21] M. Benedikt, L. Libkin, T. Schwentick, L. Segoufin, "A model-theoretic approach to regular string relations", Proceedings of the 16th IEEE Symposium on Logic in Computer Science, IEEE Computer Society Press, 2001, 431-442.

[22] M. O. Rabin, "Decidability of second-order theories and automata on infinite trees", Trans. Amer. Math. Soc., 141:7 (1969), 1-35.

[23] М. О. Рабин, "Разрешимость теорий второго порядка и автоматы над бесконечными деревьями", Кибернетический сборник, 8, Мир, М., 1971, 72-116.

C. М. Дудаков (S. M. Dudakov)

Тверской государственный университет

Поступила в редакцию

E-mail: Sergey.Dudakov@tversu.ru

M. А. Тайцлин (M. A. Taitslin)

Тверской государственный университет

E-mail: Michael.Taitslin@tversu.ru 